\title{
Uniform distribution of zero ordinates of Epstein zeta-functions
}

Dissertationsschrift zur Erlangung des naturwissenschaftlichen

Doktorgrades der Julius-Maximilians-Universität Würzburg

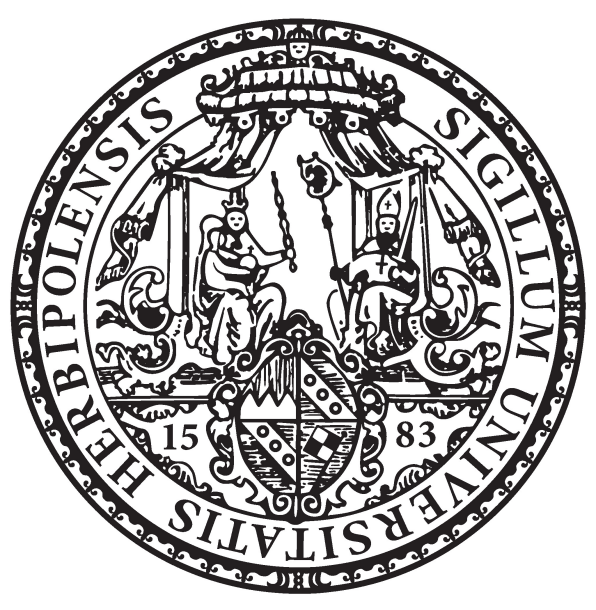

vorgelegt von

Christof Schmeller

aus

Reinheim

Würzburg 2021 
Eingereicht am 19.05.2021

bei der Fakultät für Mathematik und Informatik

der Julius-Maximilians-Universität Würzburg

1. Gutachter: Prof. Dr. Jörn Steuding

2. Gutachter: Prof. Dr. Ramūnas Garunkštis

Tag der Disputation: 02.12.2021 


\section{Contents}

Acknowledgments............................. 1

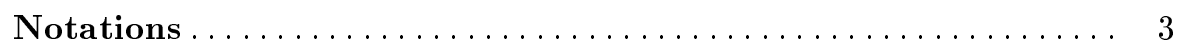

1 Introduction and statement of the main results ......... 5

1.1 The Epstein zeta-function $\ldots \ldots \ldots \ldots \ldots \ldots \ldots \ldots \ldots \ldots \ldots$

1.2 Uniform distribution modulo one $\ldots \ldots \ldots \ldots \ldots \ldots \ldots \ldots$

1.3 Landau's theorem for the Riemann zeta-function $\ldots \ldots \ldots \ldots \quad 7$

1.4 Statement of the main results and outline of the thesis ..... 7

2 Uniform distribution modulo one $\ldots \ldots \ldots \ldots \ldots \ldots \ldots \ldots, 11$

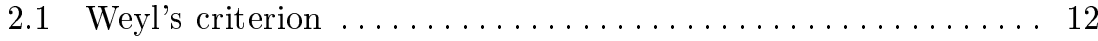

2.2 Composition of uniformly distributed sequences $\ldots \ldots \ldots \ldots \ldots 13$

2.3 Ordinates of zeros of the Riemann zeta-function ......... 13

2.4 Lemma for uniform distribution modulo one ........... 15

2.5 Counterexample for more than two weights ........... 18

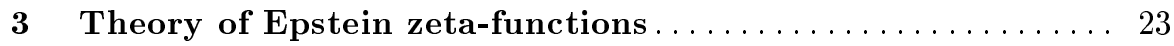

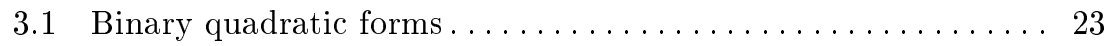

3.1 .1 Basic definitions........................ 23

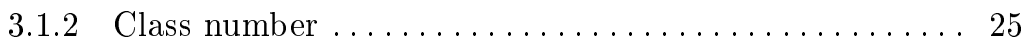

3.1.3 Connection to quadratic number fields .......... 27

3.2 Generating functions associated with forms and ideals....... 34

3.3 Epstein zeta-functions for $n \leq 2 \ldots \ldots \ldots \ldots \ldots \ldots \ldots \ldots \ldots \ldots$

3.4 Epstein zeta-functions for $n \geq 2 \ldots \ldots \ldots \ldots \ldots \ldots \ldots \ldots \ldots$

3.5 Zero distribution of Epstein zeta-functions . . . . . . . . . 41

3.5.1 Zero distribution for $n \leq 2 \ldots \ldots \ldots \ldots \ldots \ldots \ldots \ldots 41$

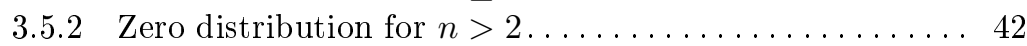




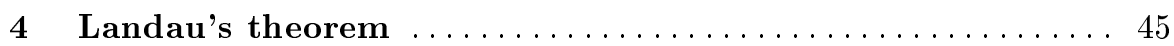

4.1 Landau's theorem in his original paper .............. 45

4.2 Applications and generalizations . . . . . . . . . . . . . 47

4.3 Uniform versions of Landau's theorem ............... 47

4.4 Uniform distribution modulo one for various zeta-functions ... 48

5 Uniform distribution of imaginary parts of zeros of

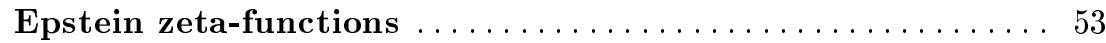

5.1 Preliminaries............................. 53

5.2 Landau's theorem for Epstein zeta-functions . . . . . . . . . . 57

5.3 Corollaries.................................. 58

5.4 Proof of the main results $\ldots \ldots \ldots \ldots \ldots \ldots \ldots \ldots \ldots \ldots$

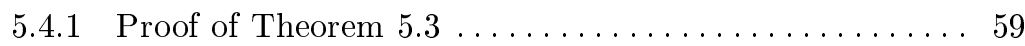

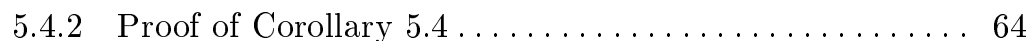

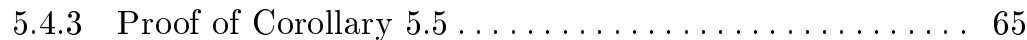

5.5 Application of the main results . . . . . . . . . . . . 66

5.5.1 The density hypothesis for binary quadratic forms .... 66

5.5.2 Application to Epstein zeta-functions for $n=4$ and

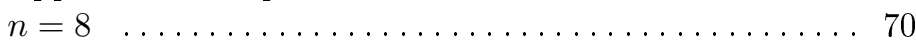

5.6 Recursion formula for the logarithmic derivative $\ldots \ldots \ldots \ldots 71$

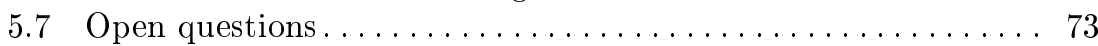

6 Discrepancy estimate for zero ordinates of Epstein

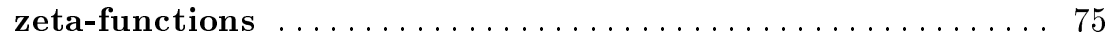

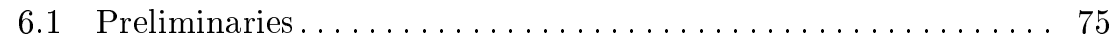

6.2 Uniform version of Theorem $5.3 \ldots \ldots \ldots \ldots \ldots \ldots \ldots \ldots 78$

6.3 Discrepancy for zero ordinates of Epstein zeta-functions . . . . 84

References $\ldots \ldots \ldots \ldots \ldots \ldots \ldots \ldots \ldots \ldots \ldots \ldots \ldots \ldots \ldots \ldots \ldots$ 


\section{Acknowledgments}

First and foremost, I want to express my deepest gratitude to my supervisor Jörn Steuding. Back in May 2008 I had a vague idea and a crazy dream to do a doctorate in Number Theory parallel to my full time work. After some rudimentary online search I contacted Jörn at the University of Würzburg. He immediately responded, we met, and he offered me various different opportunities of topics in Number Theory to pursue this dream. I was fascinated and started to work on a generalization of the famous Green-Tao Theorem on primes in arithmetic progressions. I had the opportunity to meet the protagonists of the Theorem in Budapest in 2010 at the conference of Szemerédis 70th birthday, it was great. In December 2012 the generalization I was working on was proven by a much quicker mathematician. Nevertheless the mathematics I was able to study was beautiful. Already early in 2013 Jörn offered me a new interesting topic regarding Epstein zeta-functions. In countless private lessons on Number Theory, Algebra and many related fields he supported me over a decade. Without his tremendous support this thesis would have been completely impossible. Vielen Dank lieber Jörn!

Furthermore I want to thank many nice mathematicians I had the privilege to meet in various places during seminars and conferences. Especially in Lithuania, in Vilnius and Palanga, but also in Göttingen or other places at the German Motorway A7, during the conference "'Arithmetik an der A7". I am also very grateful for the always friendly and uncomplicated atmosphere at the Departments of Mathematics in Würzburg. I found a lot of support from all doctorate students, my special thanks go to Thomas and Martin.

Last but not least, this thesis would not have been possible without the support of my loving family and friends. They encouraged me to start the journey and believed in me all the time.

Vielen Dank liebe Francesca, liebe Chiara und lieber Fabio!

Reinheim, Mai 2021

Christof Schmeller 



\section{Notations}

Here we indicate some of the notations and conventions used in this thesis, most of them are standard. However, this list is not complete; we omit notions which only appear in one section where they are defined in situ or which are standard.

As usual, we denote by $\mathbb{N}=\{1,2,3, \ldots\}$ the set of positive integers. The sets of integers, rational numbers, real numbers, and complex numbers are denoted by $\mathbb{Z}, \mathbb{Q}, \mathbb{R}$, and $\mathbb{C}$, respectively. Following an old tradition in the theory of the zeta-function, the complex variable is given by a mixture of greek and latin letters: we write $s=\sigma+i t$, where $\sigma, t \in \mathbb{R}$, and $i$ is the imaginary unit $\sqrt{-1}$ in the upper half-plane.

The letter $p$, with and without a subscript, denotes a prime number. The symbol $\equiv$ stands either for some congruence or it denotes that a function is constant. The number of elements of a finite set $\mathcal{A}$ is denoted by $\# \mathcal{A}$.

The logarithm is, as usual in number theory, always taken to the basis $e=\exp (1)$. The integer part and fractional part of a real number $x$ are indicated by $[x]$ and $\{x\}$, respectively. Very convenient is the use of the Landauand Vinogradov-symbols. Given two functions $f(x)$ and $g(x)$, both defined for $x \in X$, where $g(x)$ is positive for all $x \in X$, we write

- $f(x)=O(g(x))$ and $f(x) \ll g(x)$, respectively, if there exists a constant $C \geq 0$ such that

$$
|f(x)| \leq C g(x) \quad \text { for all } \quad x \in X
$$

here $X$ is specified either explicitly or implicitly. Usually, the set $X$ is an interval $[\xi, \infty)$ for some real number $\xi$.

- $f(x)=o(g(x))$ if the following limit exists and is equal to zero:

$$
\lim _{x \rightarrow \infty} \frac{|f(x)|}{g(x)}
$$





\title{
Introduction and statement of the main results
}

\author{
"Aller Anfang ist schwer." \\ Johann Wolfgang von Goethe (1749-1832)
}

The Epstein zeta-function is an interesting object in number theory. It can be considered as one of many different ways to generalize the Riemann zetafunction. Likewise the distribution of zeros of Epstein zeta-functions is a natural topic to study. Given its connection to quadratic forms it is no surprise that the Epstein zeta-function contains information about primes and irreducibles of number fields. Apart from the famous interest into the distribution of real parts of the zeros of zeta-functions, which leads for example to the question posed in the Riemann hypothesis, also the vertical dictribution of the zeros has been under constant scrutiny. Here uniform distribution modulo one enters the picture. In 1916 Hermann Weyl gave the famous characterization of uniform distribution via his equivalent criteria, which was amongst numerous other advances key in establishing uniform distribution modulo one for the imaginary parts of nontrivial zeros of the Riemann zeta-function decades later.

The main scope of this thesis is to investigate the wide class of Epstein zetafunctions. We want to know if the imaginary parts of nontrivial zeros are uniformly distributed modulo one.

In this introductory chapter section 1.1 introduces the Epstein zeta-function. Section 1.2 states briefly the definition of uniform distribution modulo one and section 1.3 explores Landau's theorem from 1912, which plays an important role. Section 1.4 outlines this thesis and states the main results. 


\subsection{The Epstein zeta-function}

1903 Paul Epstein [18] introduced zeta-functions associated with quadratic forms. If $\mathcal{Q}$ is a positive definite $n \times n$ matrix with integer values and $\mathcal{Q}[\mathbf{x}]=$ $\mathbf{x}^{\mathrm{t}} \mathcal{Q} \mathbf{x}$ the associated quadratic form, then

$$
\zeta(s ; \mathcal{Q})=\sum_{0 \neq \mathbf{x} \in \mathbb{Z}^{n}} \mathcal{Q}[\mathbf{x}]^{-s}
$$

is called the associated Epstein zeta-function. This Dirichlet series converges absolutely for $\operatorname{Re} s>\frac{n}{2}$ and there exists an analytic continuation to the whole complex plane except for a simple pole at $s=\frac{n}{2}$. It is also well known, see for example Steuding [65], that it satisfies the functional equation

$$
\pi^{-s} \Gamma(s) \zeta(s ; \mathcal{Q})=(\operatorname{det} \mathcal{Q})^{-\frac{1}{2}} \pi^{s-\frac{n}{2}} \Gamma\left(\frac{n}{2}-s\right) \zeta\left(\frac{n}{2}-s ; \mathcal{Q}^{-1}\right)
$$

with $\mathcal{Q}^{-1}$ the inverse matrix of $\mathcal{Q}$ and $\Gamma(s)$ the Gamma-function. The trivial zeros lie for $m \in \mathbb{N}$ at $s=-m$, though we are interested in the remaining zeros, the nontrivial ones denoted by $\varrho=\beta+i \gamma$.

Let $N(T ; \mathcal{Q})$ count the number of nontrivial zeros with $|\gamma|<T$. There exists an analogue of the Riemann von-Mangoldt formula, i.e.

$$
N(T ; \mathcal{Q})=\frac{2 T}{\pi} \log \frac{T}{\pi e \sqrt{\mathrm{m}(\mathcal{Q}) \mathrm{m}\left(\mathcal{Q}^{-1}\right)}}+O(\log T),
$$

see for example Steuding [66]. Here

$$
\mathrm{m}(\mathcal{Q})=\min \left\{m \in \mathbb{R} \mid \exists \mathbf{x} \in \mathbb{Z}^{n}: \mathcal{Q}[\mathbf{x}]=m\right\}
$$

is the minimum value attained by the quadratic form, an important invariant of $\mathcal{Q}$ frequently used in what follows.

We will explore many more properties of Epstein zeta-functions throughout this thesis but would like to close this section with a brief remark about where we find them. They are interesting analytical objects playing an important role in algebraic number theory and in the theory of modular forms, see for example Siegel [63]. We also find Epstein zeta-functions in chemistry and physics, see for instance the articles of Buhler and Crandall [8], Elizalde and Romeo [15], and Elizalde [16].

\subsection{Uniform distribution modulo one}

As far back as in the middle ages observations were made which in the modern language of mathematics can be connected to uniform distribution modulo one. For example around 1360 the French mathematician Nicole Oresme considered two bodies on a circle with uniform but incommensurable velocities and claimed that 
"No sector of a circle is so small that two such bodies could not conjunct in it at some future time, and could not have conjuncted in it some time in the past."

This can be interpreted in a way that the multiples of an irrational number lie dense in the unit interval. Centuries later the basic definition where we can build on runs as follows:

A sequence of real numbers $\left(x_{n}\right)_{n \in \mathbb{N}}$ is uniformly distributed modulo one if for every pair $\alpha, \beta$ of real numbers with $0 \leq \alpha<\beta \leq 1$, the proportion of the fractional parts of the $x_{n}$ in the half open interval $[\alpha, \beta)$ tends to its length in the following sense:

$$
\lim _{N \rightarrow \infty} \frac{\#\left\{1 \leq n \leq N:\left\{x_{n}\right\} \in[\alpha, \beta)\right\}}{N}=\beta-\alpha .
$$

\subsection{Landau's theorem for the Riemann zeta-function}

In 1912 Edmund Landau published his paper "Über die Nullstellen der Zetafunktion" [43]. In this he proved for the nontrivial zeros $\varrho=\beta+i \gamma$ of the Riemann zeta-function

$$
\zeta(s)=\sum_{n=1}^{\infty} \frac{1}{n^{s}}
$$

the following estimate: For an arbitrary real parameter $x>1$ we have

$$
\sum_{0<\gamma<T} x^{\varrho}=-\Lambda(x) \frac{T}{2 \pi}+O(\log T),
$$

where the von Mangoldt $\Lambda$-function is defined by

$$
\Lambda(n)=\left\{\begin{array}{cl}
\log p & \text { if } n=p^{k} \text { with } k \in \mathbb{N} \\
0 & \text { otherwise }
\end{array}\right.
$$

If $0<x<1, \Lambda(x)$ needs to be replaced by $x \cdot \Lambda\left(\frac{1}{x}\right)$ due to the symmetrical distribution of nontrivial zeros.

\subsection{Statement of the main results and outline of the thesis}

The main focus of this thesis is to investigate the distribution of imaginary parts of nontrivial zeros of Epstein zeta-functions. In particular we want to 
explore the subset of Epstein zeta-functions where we can show that the imaginary parts are uniformly distributed modulo one. For the Riemann zetafunction $\zeta(s)$, the simplest form of an Epstein zeta-function, Rademacher [55] proved this in 1956 under the assumption of the Riemann hypothesis. He showed that for any positive real number $z \neq 1$, the sequence $\left(\frac{\log z}{2 \pi} \gamma\right)_{\gamma}$ is uniformly distributed modulo one. Here $\gamma$ ranges through the set of positive imaginary parts of the nontrivial zeros of $\zeta(s)$ in ascending order. Unconditionally this was first shown by Elliott [17] in 1972.

In Chapter 2 we introduce the concept of uniform distribution modulo one and connect it to the question of distribution of imaginary parts of zeta-functions. The central elements to achieve this are Weyl's criterion and Landau's theorem. Usually there exist different real parts in the set of nontrivial zeros of a general Epstein zeta-function, to illustrate this we recall several results in section 3.5.

Therefore a natural question is to ask to which extent in this general case a weighted version of Weyl's criterion exists such that uniform distribution modulo one can be derived directly from a Landau type theorem. This we investigate in section 2.4 where we prove in Lemma 2.4 the following: Assuming the sequence $\tilde{\beta}_{k}=e^{2 \pi \beta_{k}}$ takes only two different values $\tilde{\beta}_{\text {min }}$ and $\tilde{\beta}_{\text {max }}$, and

$$
\lim _{N \rightarrow \infty} \frac{1}{N} \sum_{k=1}^{N}\left(\tilde{\beta}_{k}\right)^{m} \cdot e^{2 \pi m i \gamma_{k}}=0
$$

holds for all $m \in \mathbb{Z} \backslash\{0\}$, then the sequence $\left(\gamma_{k}\right)_{k \in \mathbb{N}}$ is uniformly distributed modulo one. That Lemma can help in some cases of Epstein zeta-functions to prove uniform distribution modulo one of the zero ordinates without further assumptions, we provide examples in section 5.5.2. This method comes quickly to an end though. For already three different weights $\tilde{\beta}_{k}$ we provide a counterexample in section 2.5 , i.e. a sequence which fulfills the limit conditions (1.7) without being uniformly distributed modulo one.

In Chapter 3 we look at the theory of Epstein zeta-functions and their algebraic foundation in general. We first explore fundamental properties of binary quadratic forms. For all those it will turn out that the zero ordinates of the associated Epstein zeta-function are uniformly distributed modulo one which will be shown in section 5.5.1. We recall the connection between binary quadratic forms and quadratic number fields and introduce generating functions associated with forms and ideals. We give various examples of forms where Epstein zeta-functions can be represented as a linear combination of more familiar objects like Dedekind zeta-functions and Hecke L-functions. The chapter closes with a summary of known results about the zero distribution of Epstein zeta-functions.

Chapter 4 deals with Landau's theorem in more detail. We recall some applications and generalizations of the theorem including uniform versions in both 
variables $x$ and $T$. We study in particular the result of Akbary and Murty [1], which is based on Landau's theorem for a class of Dirichlet series more general than the Selberg class, called $\tilde{\mathcal{S}}$. From this, uniform distribution modulo one of the zero ordinates is derived with help of an average density hypothesis, i.e. assuming an upper bound for the number of zeros $N_{F}(\sigma, T)$ with $F \in \mathcal{\mathcal { S }}$ in the strip $0 \leq \gamma \leq T$ and $1 / 2 \leq \sigma \leq T$. This result is important for the context of this thesis since we use a similar density hypothesis to prove a quasi equivalent result for Epstein zeta-functions in Chapter 5.

Chapter 5 contains the main results of this thesis. We prove the analogue version of Landau's theorem valid for all Epstein zeta-functions, Theorem 5.3. In essence we shall see that for an arbitrary parameter $x \in \mathbb{R}^{+} \backslash\{1\}$, as $T \rightarrow \infty$,

$$
\sum_{|\gamma|<T} x^{\varrho}=\frac{T}{\pi}\left(\delta_{l_{k}(\mathcal{Q}), x} \cdot b_{k}(\mathcal{Q})-x^{\frac{n}{2}} \delta_{l_{k}\left(\mathcal{Q}^{-1}\right), \frac{1}{x}} \cdot b_{k}\left(\mathcal{Q}^{-1}\right)\right)+O(\log T) .
$$

Here the coefficients $l_{k}(\mathcal{Q})$ and $b_{k}(\mathcal{Q})$ can be explicitly defined which we show in Lemma 5.1. They occur in the following relation of the logarithmic derivative of the slightely modified Epstein zeta-function $Z(s ; \mathcal{Q})$, defined in $(5.1)$, which carries the same zeros as $\zeta(s ; \mathcal{Q})$ :

$$
\frac{Z^{\prime}(s ; \mathcal{Q})}{Z(s ; \mathcal{Q})}=\sum_{n=1}^{\infty} \frac{b_{n}(\mathcal{Q})}{l_{n}(\mathcal{Q})^{s}}
$$

For these results we use ideas of Steuding ([67], [65]) and evaluate a contour integral of $\frac{Z^{\prime}(s ; \mathcal{Q})}{Z(s ; \mathcal{Q})}$. Some added complexity stems from the fact that for the logarithmic derivative of the general Epstein zeta-function we have to deal with a general Dirichlet series where the coefficients are related to non integer values. By demanding the density hypothesis

$$
\sum_{|\gamma|<T}\left|\beta-\frac{n}{4}\right|=o(N(T ; \mathcal{Q}))
$$

to the imaginary parts of the nontrivial zeros we conclude uniform distribution modulo one of the zero ordinates, see Corollary 5.4. In section 5.5.1 we apply this result to the case where a binary quadratic form is governing the Epstein zeta-function. We are able to show that the density hypothesis is fulfilled by using methods from Levinson [47], Steuding (see [65],[69]) and the meansquare estimate

$$
\int_{0}^{T}\left|\zeta\left(\frac{1}{2}+i t ; \mathcal{Q}\right)\right|^{2} d t=C(\mathcal{Q}) T(\log T)^{2}+O(T \log T)
$$

holding with some constant $C(\mathcal{Q})$, which was shown by Müller [49]. Leveraging Lemma 2.4 we show in Corollary 5.5 that uniform distribution modulo one can 
also be derived for the zero ordinates, if the real parts of zeros cluster around two different lines. Examples of Epstein zeta-functions where the associated quadratic form carries more than two variables and for which this is valid are given in section 5.5.2. We conclude the chapter with an alternative recursive representation of the logarithmic derivative of $Z(s ; \mathcal{Q})$.

In Chapter 6 we approach a quantitative view of the distribution of nontrivial zero ordinates of Epstein zeta-functions. The discrepancy measures how good a sequence is distributed modulo one. We postulate the natural condition of a clustering of zeros around the critical line, i.e. (1.8) at a certain speed. In particular we demand $\sum_{|\gamma|<T}\left|\beta-\frac{n}{4}\right|=O\left(\frac{T \log T}{\log \log T}\right)$, which holds for the Riemann zeta-function as shown by Levinson [47]. Then we shall see that the estimate for the discrepancy $D_{T}$ for Epstein zeta-functions is

$$
D_{T} \ll \frac{|\log z|}{\log \log \log T} .
$$

for all positive $z \neq 1$. To prove this we apply the Theorem of Erdös and Turán while using ideas of Rehberg [56], as well as Baluyot and Gonek [3].

\section{So what is really new?}

Lemma 2.4 gives a new possibility to derive uniform distribution modulo one for any sequence satisfying the stated limit conditions with two arbitrary weights. Further the construction of the counterexample in section 2.5 for three weights. Theorem 5.3 generalizes Landau's theorem for all Epstein zetafunctions. Corollaries 5.4 and 5.5 provide the opportunity to apply it to a certain class of Epstein zeta-functions. The recursive representation arose from the analysis of Theorem 5.3 and the respective Lemma 5.1. Finally the discrepancy estimate in Chapter 6 is new. The proof uses certain properties of Epstein zeta-functions represented by general Dirichlet series. 


\title{
Uniform distribution modulo one
}

\author{
"Besides language and music mathematics is one of the primary \\ manifestations of the free creative power of the human mind."
}

Hermann Weyl (1885-1955)

In this chapter we introduce uniform distribution modulo one from the point of view of this thesis in more detail. We start by stating Weyl's criterion in section 2.1. In section 2.3 we show how it is used to prove uniform distribution modulo one of the Riemann zeta zero ordinates by applying well-known results of Levinson [47] about the clustering of the zeros around the critical line. Moreover, we recall in section 2.2 a result of Akbary and Murty [1], which highlights that the ordered finite union of uniform distributed sequences modulo one is again uniformly distributed modulo one. This can later be applied in section 5.5 when we investigate more general Epstein zeta-functions.

In section 2.4 we explore Weyl's criterion for our purpose by allowing powers of different weights to appear. We prove that if just two arbitrary real numbers occur as weights in the exponential sum, this yields another criterion for uniform distribution modulo one. Moreover, in section 2.5 we see on the other hand that for powers of three different weights there exists a counterexample of a sequence obeying the limit conditions, but not being uniformly distributed modulo one. Essentially this is because the third weight adds another degree of freedom. We construct a sequence which approximates three different density functions with non-zero Fourier coefficients and still obeys all limit conditions. We conclude that in case of more than two different real parts occurring in the set of nontrivial zeros more information about the zetafunctions is required in order to derive uniform distribution modulo one for their zero ordinates. 


\subsection{Weyl's criterion}

In his famous articles [78], [79] and [80] Hermann Weyl developed an important criterion which is equivalent to uniform distribution modulo one as defined in (1.5). He first proved the following

Theorem 2.1. The sequence $\left(x_{n}\right)_{n \in \mathbb{N}}$ is uniformly distributed modulo one if, and only if, for any Riemann integrable function $f:[0,1] \rightarrow \mathbb{C}$

$$
\lim _{N \rightarrow \infty} \frac{1}{N} \sum_{n=1}^{N} f\left(\left\{x_{n}\right\}\right)=\int_{0}^{1} f(x) d x .
$$

The proof, see for example the classic reference on uniform distribution from Kuipers and Niederreiter [42], uses the indicator function $\mathbf{1}_{[\alpha, \beta)}$ and the fact that $\int_{0}^{1} \mathbf{1}_{[\alpha, \beta)}(x) d x=\beta-\alpha$ holds obviously. Linear combinations of those indicator functions lead to the statement for step and finally for all Riemann integrable functions. This brings us to the celebrated Weyl criterion which can be stated as follows:

Theorem 2.2 (Weyl's criterion). The sequence $\left(x_{n}\right)_{n \in \mathbb{N}}$ is uniformly distributed modulo one if, and only if, for all $m \in \mathbb{Z} \backslash\{0\}$

$$
\lim _{N \rightarrow \infty} \frac{1}{N} \sum_{n=1}^{N} e^{2 \pi m i x_{n}}=0 .
$$

To prove this Weyl utilized the Weierstrass approximation theorem for trigonometric polynomials and Theorem 2.1 above; see for instance again Kuipers and Niederreiter [42], Theorem 2.1.

Weyl's criterion is often used to establish uniform distribution modulo one and was applied and extended in various ways. Just to name a few, Weyl himself extended results on sequences of the form $n^{k} \alpha$ with irrational $\alpha$, mathematical billards and the three body problem, see [79]. Also Vinogradov [76] applied it in his proof of the ternary Goldbach conjecture, which states that any sufficiently large odd integer can be represented as a sum of three primes.

One of many applications of criterion 2.2 was to investigate the distribution of zero ordinates of first the Riemann zeta-function and later zeta-functions in general. The structure of Landau's theorem (1.6) with the free parameter $x$ shows similarities to the exponential sum in Weyl's theorem and we will consider the connection in the next section.

But first we explore a simple possibility to derive uniform distribution modulo one for unions of sequences which are already uniformly distributed. 


\subsection{Composition of uniformly distributed sequences}

Akbary and Murty showed in [1], proposition 12 the following consequence of Weyl's criterion.

Theorem 2.3. i) Let $\left(a_{n}\right)_{n \in \mathbb{N}}$ and $\left(b_{n}\right)_{n \in \mathbb{N}}$ be two monotonically increasing sequences of positive real numbers and let $\left(c_{n}\right)_{n \in \mathbb{N}}$ be the union of these two sequences ordered according to the absolute value of its elements. If $\left(a_{n}\right)_{n \in \mathbb{N}}$ and $\left(b_{n}\right)_{n \in \mathbb{N}}$ are uniformly distributed modulo one, then $\left(c_{n}\right)_{n \in \mathbb{N}}$ is also uniformly distributed modulo one.

ii) Let $F, G$ be general Dirichlet series. If the sequences $\left(\alpha \gamma_{F, n}\right)_{n \in \mathbb{N}}$ and $\left(\alpha \gamma_{G, n}\right)_{n \in \mathbb{N}}$, with $\alpha \neq 0$, formed from imaginary parts of zeros of $F$ and $G$ are uniformly distributed modulo one, then the same is true for the sequence $\left(\alpha \gamma_{F G, n}\right)_{n \in \mathbb{N}}$ of imaginary parts of zeros of $F \cdot G$.

Proof: With $t>0$ let $n_{c}(t)$ be the number of elements of $\left(c_{n}\right)_{n \in \mathbb{N}}$ not exceeding $t$. Hence $n_{c}(t)=n_{a}(t)+n_{b}(t)$. By denoting a general term of $\left(c_{n}\right)_{n \in \mathbb{N}}$ with $c$ we get

$$
\begin{aligned}
\frac{\left|\sum_{c \leq t} e^{2 \pi i m c}\right|}{n_{c}(t)} & =\frac{\left|\sum_{a \leq t} e^{2 \pi i m a}+\sum_{b \leq t} e^{2 \pi i m b}\right|}{n_{a}(t)+n_{b}(t)} \\
& \leq \frac{\left|\sum_{a \leq t} e^{2 \pi i m a}\right|}{n_{a}(t)}+\frac{\left|\sum_{b \leq t} e^{2 \pi i m b}\right|}{n_{b}(t)} .
\end{aligned}
$$

Therefore (i) follows from Weyl's criterion. (ii) is straightforward from (i), since the set of zeros of $F \cdot G$ is the union of the set of zeros of $F$ and the set of zeros of $G$.

For our purpose this simple result is very useful since it transfers uniform distribution modulo one to products of zeta-functions. We will return to this fact when we discuss more general Dirichlet series.

\subsection{Ordinates of zeros of the Riemann zeta-function}

We first consider how Landau's theorem (1.6) and Weyl's criterion Theorem 2.2 play together in case of the Riemann zeta-function. For this we need an asymptotic formula about the number of nontrivial zeros $\varrho=\beta+i \gamma$ up to a given height $T$, denoted by $N(T)$. As conjectured by Riemann and famously proven by von Mangoldt in 1895, the Riemann von-Mangoldt formula gives

$$
N(T)=\frac{T}{2 \pi} \log \frac{T}{2 \pi e}+O(\log T)
$$

Now starting with Landau's theorem (1.6), dividing by the number of nontrivial zeros using (2.3) above, this yields for $x>1$ 


$$
\frac{1}{N(T)} \sum_{0<\gamma<T} x^{\varrho} \ll \frac{\log x}{\log T} .
$$

Next we aim to leverage results of the clustering of zeros around the critical line. Therefore we observe that

$$
\begin{aligned}
\left|x^{\left(\frac{1}{2}+i \gamma\right)}-x^{(\beta+i \gamma)}\right| & =\left|x^{i \gamma}\right|\left|x^{\frac{1}{2}}-x^{\beta}\right| \\
& =\left|x^{\beta}\left(x^{\left(\frac{1}{2}-\beta\right)}-1\right)\right| \\
& \leq x^{\beta}\left|\exp \left(\left(\frac{1}{2}-\beta\right) \log x\right)-1\right| \\
& \leq\left|\beta-\frac{1}{2}\right||\log x| \max \left\{x^{\beta}, x^{\frac{1}{2}}\right\} .
\end{aligned}
$$

Hence

$$
\begin{aligned}
\frac{1}{N(T)} & \sum_{0<\gamma<T}\left|x^{\left(\frac{1}{2}+i \gamma\right)}-x^{(\beta+i \gamma)}\right| \\
\leq & \frac{\max \left\{x^{\beta}, x^{\frac{1}{2}}\right\}|\log x|}{N(T)} \sum_{0<\gamma<T}\left|\beta-\frac{1}{2}\right|
\end{aligned}
$$

To proceed we need an estimate for $\sum_{0<\gamma<T}\left|\beta-\frac{1}{2}\right|$. Without assuming the Riemann hypothesis we could now use the following results. Littlewood [48] showed that

$$
\sum_{0<\gamma \leq T}\left|\beta-\frac{1}{2}\right| \ll T \log \log T .
$$

Selberg [61] improved on this by integration of his density theorem getting

$$
\sum_{0<\gamma \leq T}\left|\beta-\frac{1}{2}\right| \ll T .
$$

Weaker than those two results but still a sufficient asymptotic formula was proven by Levinson [47]. It has the advantage that it can be generalized to other zeta-functions. We will use it since this is more in the scope of what follows. His result was dealing with general $a$-points of the Riemann zetafunction. Applied to the nontrivial zeros, Levinson showed

$$
\sum_{0<\gamma \leq T}\left|\beta-\frac{1}{2}\right| \ll T(\log \log T)^{2} .
$$

Putting (2.6) into (2.5) and using (2.3), we obtain 


$$
\frac{1}{N(T)} \sum_{0<\gamma \leq T}\left(x^{\left(\frac{1}{2}+i \gamma\right)}-x^{(\beta+i \gamma)}\right) \ll \frac{(\log \log T)^{2}}{\log T} .
$$

Here and in the following the implied constant depends on the parameter $x$. Bearing in mind that (2.4) holds we can further conclude

$$
\frac{1}{N(T)} \sum_{0<\gamma \leq T} x^{\left(\frac{1}{2}+i \gamma\right)} \ll \frac{(\log \log T)^{2}}{\log T} .
$$

Now setting the free parameter $x=z^{m}$ for some real number $z>1$ and $m \in \mathbb{Z} \backslash\{0\}$ we see that

$$
\frac{1}{N(T)} \sum_{0<\gamma \leq T} e^{(\log z \cdot m i \gamma)} \ll \frac{(\log \log T)^{2}}{\log T} .
$$

For $T \rightarrow \infty$ this tends to zero, so according to Weyl's criterion 2.2 the sequence of numbers $\alpha \gamma$ with $\alpha=\frac{\log z}{2 \pi}$ is uniformly distributed modulo one.

\subsection{Lemma for uniform distribution modulo one}

In this section we want to explore to what extent we can draw a conclusion about the distribution of a sequence already from a formula like (2.4). It is a natural question in our context since analogous results exist for many zeta-functions. In Chapter 3 we will investigate those variants in more detail.

We start with the Landau type formula (2.4) and denote by $N=N(T)$ the number of zeros up to a given height $T$. Since the right-hand side of (2.4) tends to zero as $T \rightarrow \infty$, we can write

$$
\lim _{N \rightarrow \infty} \frac{1}{N} \sum_{k=1}^{N} x^{\beta_{k}+i \gamma_{k}}=0
$$

which holds for $x \in \mathbb{R}^{+} \backslash\{1\}$. Then, by setting $x=e^{2 \pi m}$, we obtain for all $m \in \mathbb{Z} \backslash\{0\}$

$$
\lim _{N \rightarrow \infty} \frac{1}{N} \sum_{k=1}^{N} e^{2 \pi m \beta_{k}} \cdot e^{2 \pi m i \gamma_{k}}=0 .
$$

Without adding any hypothesis on the distribution of the real parts $\beta_{k}$ of the nontrivial zeros, we consider in the following what this implies already for the distribution of the imaginary parts $\gamma_{k}$. In particular, we prove that if there are only two different values for the real parts, say $\beta_{\min }$ and $\beta_{\max }$, then the imaginary parts are uniformly distributed modulo one. 
Nevertheless for already three different real parts of zeros this is not the case anymore in general if no further assumptions are made for the real parts $\beta_{k}$. In order to show this we provide in section 2.5 an explicit example of a sequence where the imaginary parts $\gamma_{k}$ are not uniformly distributed modulo one, although (2.7) holds for all $m \in \mathbb{Z} \backslash\{0\}$.

Lemma 2.4. Assume the sequence $\tilde{\beta}_{k}=e^{2 \pi \beta_{k}}$ takes only two different real values $\tilde{\beta}_{\min }, \tilde{\beta}_{\max }$ and let

$$
\lim _{N \rightarrow \infty} \frac{1}{N} \sum_{k=1}^{N}\left(\tilde{\beta}_{k}\right)^{m} \cdot e^{2 \pi m i \gamma_{k}}=0
$$

for all $m \in \mathbb{Z} \backslash\{0\}$. Then the imaginary parts $\gamma_{k}$ are uniformly distributed modulo one.

Proof: We assume that the $\gamma_{k}$ are not uniformly distributed modulo one. Then there exists an $\tilde{m} \in \mathbb{Z} \backslash\{0\}$ and an accumulation point not equal to zero, i.e. a sequence of natural numbers $N_{1}, N_{2} \ldots$ and an $\alpha \in \mathbb{C}$ with $\alpha \neq 0$ such that

$$
\lim _{i \rightarrow \infty} \frac{1}{N_{i}} \sum_{k=1}^{N_{i}} e^{2 \pi \tilde{m} i \gamma_{k}}=\alpha .
$$

Now we consider (2.8) for the values $\tilde{m}$ and $-\tilde{m}$ and split the sum into two parts where $\gamma_{k}$ is an imaginary part belonging to either $\tilde{\beta}_{\text {min }}$ and $\tilde{\beta}_{\text {max }}$. Hence, we get

$$
\lim _{N \rightarrow \infty}\left(\left(\tilde{\beta}_{\text {min }}\right)^{\tilde{m}} \frac{1}{N} \sum_{\substack{k=1 \\ \tilde{\beta}_{k}=\tilde{\bar{\beta}}_{\text {min }}}}^{N} e^{2 \pi \tilde{m} i \gamma_{k}}+\left(\tilde{\beta}_{\text {max }}\right)^{\tilde{m}} \frac{1}{N} \sum_{\substack{k=1 \\ \tilde{\beta}_{k}=\overline{\bar{\beta}}_{\text {max }}}}^{N} e^{2 \pi \tilde{m} i \gamma_{k}}\right)=0
$$

and

$$
\lim _{N \rightarrow \infty}\left(\left(\tilde{\beta}_{\min }\right)^{-\tilde{m}} \frac{1}{N} \sum_{\substack{k=1 \\ \tilde{\beta}_{k}=\tilde{\tilde{\beta}}_{\text {min }}}}^{N} e^{-2 \pi \tilde{m} i \gamma_{k}}+\left(\tilde{\beta}_{\max }\right)^{-\tilde{m}} \frac{1}{N} \sum_{\substack{k=1 \\ \tilde{\beta}_{k}=\tilde{\tilde{\beta}}_{m a x}}}^{N} e^{-2 \pi \tilde{m} i \gamma_{k}}\right)=0
$$

We observe that by defining for all $N \in \mathbb{N}$ the complex numbers

$$
\gamma_{m i n}^{N}=: \frac{1}{N} \sum_{\substack{k=1 \\ \beta_{k}=\tilde{\tilde{\beta}}_{m i n}}}^{N} e^{2 \pi \tilde{m} i \gamma_{k}}
$$

and 


$$
\gamma_{\max }^{N}=: \frac{1}{N} \sum_{\substack{k=1 \\ \beta_{k}=\tilde{\tilde{\beta}}_{\text {max }}}}^{N} e^{2 \pi \tilde{m} i \gamma_{k}}
$$

the equations above become

$$
\lim _{N \rightarrow \infty}\left(\left(\tilde{\beta}_{\min }\right)^{\tilde{m}} \gamma_{\min }^{N}+\left(\tilde{\beta}_{\max }\right)^{\tilde{m}} \gamma_{\max }^{N}\right)=0
$$

and

$$
\lim _{N \rightarrow \infty}\left(\left(\tilde{\beta}_{\min }\right)^{-\tilde{m}} \overline{\gamma_{\min }^{N}}+\left(\tilde{\beta}_{\max }\right)^{-\tilde{m}} \overline{\gamma_{\max }^{N}}\right)=0 .
$$

Therefore, if we separate $\gamma_{\min }^{N}$ and $\gamma_{\max }^{N}$ into real and imaginary parts with $\gamma_{m i n}^{N}=: \gamma_{\text {real }}^{\min , N}+i \gamma_{i m g}^{\min , N}$ and $\gamma_{\max }^{N}=: \gamma_{\text {real }}^{\max , N}+i \gamma_{i m g}^{\max , N}$, we find for all $\epsilon_{1}>0$ an $N_{0}\left(\epsilon_{1}\right) \in \mathbb{N}$ such that for all $N \geq N_{0}\left(\epsilon_{1}\right)$

$$
\begin{aligned}
& -\epsilon_{1}<\left(\tilde{\beta}_{\text {min }}\right)^{-\tilde{m}} \gamma_{\text {real }}^{\text {min }, N}+\left(\tilde{\beta}_{\text {max }}\right)^{-\tilde{m}} \gamma_{\text {real }}^{\text {max }, N}<+\epsilon_{1} \\
& -\epsilon_{1}<\left(\tilde{\beta}_{\text {min }}\right)^{\tilde{m}} \gamma_{\text {real }}^{\text {min }, N}+\left(\tilde{\beta}_{\text {max }}\right)^{\tilde{m}} \gamma_{\text {real }}^{\text {max }, N}<+\epsilon_{1},
\end{aligned}
$$

as well as for the imaginary parts

$$
\begin{aligned}
& -\epsilon_{1}<\left(\tilde{\beta}_{\text {min }}\right)^{-\tilde{m}} \gamma_{i m g}^{\min , N}+\left(\tilde{\beta}_{\max }\right)^{-\tilde{m}} \gamma_{i m g}^{\max , N}<+\epsilon_{1} \\
& -\epsilon_{1}<\left(\tilde{\beta}_{\text {min }}\right)^{\tilde{m}} \gamma_{i m g}^{\text {min }, N}+\left(\tilde{\beta}_{\max }\right)^{\tilde{m}} \gamma_{i m g}^{\max , N}<+\epsilon_{1} .
\end{aligned}
$$

Equations (2.10) can be written in matrix form as

$$
\left(\begin{array}{c}
-\epsilon_{1} \\
-\epsilon_{1}
\end{array}\right)<\left(\begin{array}{cc}
\left(\tilde{\beta}_{\text {min }}\right)^{-\tilde{m}} & \left(\tilde{\beta}_{\text {max }}\right)^{-\tilde{m}} \\
\left(\tilde{\beta}_{\text {min }}\right)^{\tilde{m}} & \left(\tilde{\beta}_{\text {max }}\right)^{\tilde{m}}
\end{array}\right)\left(\begin{array}{c}
\gamma_{\text {reaal }, N}^{\text {min }} \\
\gamma_{\text {real }}^{\text {max }}
\end{array}\right)<\left(\begin{array}{c}
+\epsilon_{1} \\
+\epsilon_{1}
\end{array}\right) .
$$

The occurring matrix

$$
B=:\left(\begin{array}{cc}
\left(\tilde{\beta}_{\text {min }}\right)^{-\tilde{m}} & \left(\tilde{\beta}_{\text {max }}\right)^{-\tilde{m}} \\
\left(\tilde{\beta}_{\text {min }}\right)^{\tilde{m}} & \left(\tilde{\beta}_{\text {max }}\right)^{\tilde{m}}
\end{array}\right)
$$

is invertible with

$$
B^{-1}=\frac{1}{\left(\tilde{\beta}_{\text {min }}\right)^{-\tilde{m}}\left(\tilde{\beta}_{\text {max }}\right)^{\tilde{m}}-\left(\tilde{\beta}_{\text {min }}\right)^{\tilde{m}}\left(\tilde{\beta}_{\text {max }}\right)^{-\tilde{m}}}\left(\begin{array}{cc}
\left(\tilde{\beta}_{\text {max }}\right)^{\tilde{m}} & -\left(\tilde{\beta}_{\text {max }}\right)^{-\tilde{m}} \\
-\left(\tilde{\beta}_{\text {min }}\right)^{\tilde{m}} & \left(\tilde{\beta}_{\text {min }}\right)^{-\tilde{m}} .
\end{array}\right)
$$

Therefore, with the usual matrix norm of absolute row sums, i.e.

$$
\|A\|_{\infty}:=\max _{i} \sum_{j=1}^{n}\left|a_{i j}\right|
$$

we obtain from (2.10) the inequalities 


$$
\begin{aligned}
& -\epsilon_{1} \cdot\left\|B^{-1}\right\|_{\infty}<\gamma_{\text {real }}^{\text {min }, N}<+\epsilon_{1} \cdot\left\|B^{-1}\right\|_{\infty} \\
& -\epsilon_{1} \cdot\left\|B^{-1}\right\|_{\infty}<\gamma_{\text {real }}^{\text {max }, N}<+\epsilon_{1} \cdot\left\|B^{-1}\right\|_{\infty} .
\end{aligned}
$$

Now looking at (2.9) we assume without loss of generality that the real part $\alpha_{\text {real }}$ of the accumulation point $\alpha \in \mathbb{C}$ is nonzero. Hence, we obtain

$$
\lim _{i \rightarrow \infty} \frac{1}{N_{i}} \sum_{k=1}^{N_{i}} e^{2 \pi \tilde{m} i \gamma_{k}}=\lim _{i \rightarrow \infty}\left(\gamma_{\text {real }}^{\text {min, } N_{i}}+\gamma_{\text {real }}^{\max , N_{i}}\right)=\alpha_{\text {real }} \neq 0
$$

That means for all $\epsilon_{2}>0$ and for all $n \in \mathbb{N}$, in particular also for $N_{0}\left(\epsilon_{1}\right)$, there exists an $N_{1} \in \mathbb{N}$ with $N_{1}>N_{0}\left(\epsilon_{1}\right)$ such that

$$
\alpha_{\text {real }}-\epsilon_{2}<\gamma_{\text {real }}^{\min , N_{1}}+\gamma_{\text {real }}^{\max , N_{1}}<\alpha_{\text {real }}+\epsilon_{2}
$$

Setting $\epsilon_{2}=\frac{\left|\alpha_{\text {real }}\right|}{4}$ we get

$$
\left|\frac{3}{4} \cdot \alpha_{\text {real }}\right|<\left|\gamma_{\text {real }}^{\min , N_{1}}+\gamma_{\text {real }}^{\max , N_{1}}\right|<\left|\frac{5}{4} \cdot \alpha_{\text {real }}\right| .
$$

But now by choosing in (2.11)

$$
\epsilon_{1}=\frac{\left|\alpha_{\text {real }}\right|}{4 \cdot\left\|B^{-1}\right\|_{\infty}}
$$

we see that $\left|\gamma_{\text {real }}^{\text {min, } N}\right|<\left|\frac{\alpha_{\text {real }}}{4}\right|$ and $\left|\gamma_{\text {real }}^{\text {max }, N}\right|<\left|\frac{\alpha_{\text {real }}}{4}\right|$, which implies

$$
\left|\gamma_{\text {real }}^{\min , N_{1}}+\gamma_{\text {real }}^{\max , N_{1}}\right|<\left|\frac{1}{2} \cdot \alpha_{\text {real }}\right| \text {. }
$$

This gives a contradiction to (2.12) and concludes the proof of the Lemma.

An application of Lemma 2.4 to Epstein zeta-functions is provided in section 5.5.2. We will now continue to show that for three or more weights a statement like Lemma 2.4 is wrong.

\subsection{Counterexample for more than two weights}

The situation changes if more than two different weights occur in (2.8). In particular, we can construct a sequence $\left(\beta_{k}+i \gamma_{k}\right)_{k \in \mathbb{N}}$ where the $\beta_{k}$ take on three different values $\beta_{1}, \beta_{2}, \beta_{3}$ and $\left\{\gamma_{k}\right\}$ is not uniformly distributed modulo one, although (2.8) holds for all $m \in \mathbb{Z} \backslash\{0\}$. Our idea is to approximate three different densities $f_{1}, f_{2}, f_{3}$ of absolutely continuous distribution functions with certain Fourier coefficients. We construct an overall sequence with 
equally one third of its elements approximating $f_{1}, f_{2}, f_{3}$ which carry different weights. The functions $f_{i}: \mathbb{T} \rightarrow \mathbb{C}$, where $\mathbb{T}$ denotes as usual the unit interval, should have the property that their only nonzero Fourier coefficients appear for values $m=0,+1,-1$. This will ensure that for values $m \notin\{+1,-1\}$ equation (2.8) holds automatically for any linear combination of the $f_{i}$.

We first recall the definition of an absolutely continuous distribution function and its density. The terminology stems from probability theory but can be applied to uniform distribution theory, see for instance section 1.6. and 1.7. in Strauch and Porubsky [70].

A non-decreasing function $g([0,1]) \rightarrow[0,1]$ with $g(0)=0$ and $g(1)=1$ is called a distribution function of the sequence $x_{n} \bmod 1$ if an increasing sequence of positive integers $N_{1}, N_{2}, \ldots$ exists such that for any $0 \leq x \leq 1$ the equality

$$
g(x)=\lim _{k \rightarrow \infty} \frac{A\left([0, x) ; N_{k} ; x_{n} \bmod 1\right)}{N_{k}}
$$

holds at every point $x$ of continuity of $g(x)$ and therefore almost everywhere on $[0,1]$. Here $A$ denotes, for any subset $I$ of $[0,1]$, the usual counting function defined by

$$
A\left(I ; N ; x_{n} \bmod 1\right):=\#\left\{1 \leq n \leq N:\left\{x_{n}\right\} \in I\right\}
$$

The following theorem describes the decomposition of any distribution function.

Theorem 2.5 (Lebesgue decomposition theorem). Any distribution function $g(x)$ can be uniquely expressed as

$$
g(x)=\alpha_{1} g_{d}(x)+\alpha_{2} g_{s}(x)+\alpha_{3} g_{a c}(x)
$$

where $\alpha_{1}, \alpha_{2}, \alpha_{3}$ are non-negative constants, $\alpha_{1}+\alpha_{2}+\alpha_{3}=1$, and

(i) $g_{d}(x)$ is a discrete distribution function, i.e. $\sum_{t_{n}<x} h_{n}$, where $t_{n}$ is the sequence of points of discontinuity of $g(x)$ with jumps $h_{n}$ at these points,

(ii) $g_{s}(x)$ is a singular distribution function, i.e. continuous, strictly increasing and having zero derivative almost everywhere,

(iii) $g_{a c}(x)$ is an absolute continuous distribution function, i.e. $g_{a c}=$ $\int_{0}^{x} h(t) d t$ for some non-negative Lebesgue integrable function $h(t)$ such that $\int_{0}^{1} h(t) d t=1$. The function $h(t)$ is called the density of $g_{a c}(x)$.

For a proof see for example Kolmogorov and Fomin [41].

In our construction we only consider the case with $\alpha_{3}=1$, i.e. the distribution function of the sequence consists only of the absolute continuous part with a density. 
Now starting in this setup with (2.8) we obtain for $m=-1,+1$

$$
\begin{array}{r}
\lim _{N \rightarrow \infty}\left(\beta_{1}^{-1} \frac{1}{N} \sum_{\substack{k=1 \\
\beta_{k}=\beta_{1}}}^{N} e^{-2 \pi i \gamma_{k}}+\beta_{2}^{-1} \frac{1}{N} \sum_{\substack{k=1 \\
\beta_{k}=\beta_{2}}}^{N} e^{-2 \pi i \gamma_{k}}+\right. \\
\left.+\beta_{3}^{-1} \frac{1}{N} \sum_{\substack{k=1 \\
\beta_{k}=\beta_{3}}}^{N} e^{-2 \pi i \gamma_{k}}\right)=0
\end{array}
$$

and

$$
\lim _{N \rightarrow \infty}\left(\beta_{1} \frac{1}{N} \sum_{\substack{k=1 \\ \beta_{k}=\beta_{1}}}^{N} e^{2 \pi i \gamma_{k}}+\beta_{2} \frac{1}{N} \sum_{\substack{k=1 \\ \beta_{k}=\beta_{2}}}^{N} e^{2 \pi i \gamma_{k}}+\beta_{3} \frac{1}{N} \sum_{\substack{k=1 \\ \beta_{k}=\beta_{3}}}^{N} e^{2 \pi i \gamma_{k}}\right)=0 .
$$

Given we had approximated the densities $f_{1}, f_{2}, f_{3}, f_{i}([0,1]) \rightarrow[0,1]$, for the distribution of the imaginary parts, we get from that the following equations for $m=-1,+1$ :

$$
\begin{aligned}
& \lim _{N \rightarrow \infty}\left(\beta_{1}^{-1} \frac{1}{N} \sum_{\substack{k=1 \\
\beta_{k}=\beta_{1}}}^{N} f_{1}\left(\left\{\gamma_{k}\right\}\right) e^{-2 \pi i \gamma_{k}}+\beta_{2}^{-1} \frac{1}{N} \sum_{\substack{k=1 \\
\beta_{k}=\beta_{2}}}^{N} f_{2}\left(\left\{\gamma_{k}\right\}\right) e^{-2 \pi i \gamma_{k}}+\right. \\
& \left.+\beta_{3}^{-1} \frac{1}{N} \sum_{\substack{k=1 \\
\beta_{k}=\beta_{3}}}^{N} f_{3}\left(\left\{\gamma_{k}\right\}\right) e^{-2 \pi i \gamma_{k}}\right)=0, \\
& \lim _{N \rightarrow \infty}\left(\beta_{1} \frac{1}{N} \sum_{\substack{k=1 \\
\beta_{k}=\beta_{1}}}^{N} f_{1}\left(\left\{\gamma_{k}\right\}\right) e^{2 \pi i \gamma_{k}}+\beta_{2} \frac{1}{N} \sum_{\substack{k=1 \\
\beta_{k}=\beta_{2}}}^{N} f_{2}\left(\left\{\gamma_{k}\right\}\right) e^{2 \pi i \gamma_{k}}+\right. \\
& \left.+\beta_{3} \frac{1}{N} \sum_{\substack{k=1 \\
\beta_{k}=\beta_{3}}}^{N} f_{3}\left(\left\{\gamma_{k}\right\}\right) e^{2 \pi i \gamma_{k}}\right)=0 .
\end{aligned}
$$

Therefore assuming convergence of the Fourier coefficients, this yields

$$
\begin{aligned}
& \beta_{1}^{-1} \widehat{f}_{1}(-1)+\beta_{2}^{-1} \widehat{f}_{2}(-1)+\beta_{3}^{-1} \widehat{f}_{3}(-1)=0 \\
& \beta_{1} \cdot \widehat{f}_{1}(+1)+\beta_{2} \cdot \widehat{f}_{2}(+1)+\beta_{3} \cdot \widehat{f}_{3}(+1)=0 .
\end{aligned}
$$

By setting $\widehat{f}_{i}(+1)=\widehat{f}_{i}(-1):=c_{i} \in \mathbb{R}$ we ensure that the density distribution functions $f_{i}$ become real valued. Postulating $\widehat{f}_{i}(0)=1$ for the Fourier coefficients yields $\int_{0}^{1} f_{i}(t) d t=1$, which is necessary to obtain proper densities. 
Hence, the aim is to find solutions to the system above where the resulting functions are non-negative. Now (2.13) is simply the linear system

$$
\left(\begin{array}{ccc}
\beta_{1}^{-1} & \beta_{2}^{-1} & \beta_{3}^{-1} \\
\beta_{1} & \beta_{2} & \beta_{3}
\end{array}\right)\left(\begin{array}{l}
c_{1} \\
c_{2} \\
c_{3}
\end{array}\right)=\left(\begin{array}{l}
0 \\
0
\end{array}\right) .
$$

For a free parameter $t \in \mathbb{R}$ the solution to this system is given by

$$
\left(\begin{array}{l}
c_{1} \\
c_{2} \\
c_{3}
\end{array}\right)=\left(\begin{array}{c}
t \\
\frac{1}{\beta_{2}^{-1} \beta_{3}-\beta_{2} \beta_{3}^{-1}} t \cdot\left(\beta_{3}^{-1} \beta_{1}-\beta_{3} \beta_{1}^{-1}\right) \\
\frac{1}{\beta_{2}^{-1} \beta_{3}-\beta_{2} \beta_{3}^{-1}} t \cdot\left(\beta_{1}^{-1} \beta_{2}-\beta_{1} \beta_{2}^{-1}\right)
\end{array}\right) .
$$

Taking as an example $\beta_{1}=1, \beta_{2}=2, \beta_{3}=3$ and choosing $t=\frac{1}{10}$ we get $c_{1}=\frac{1}{10}, c_{2}=-\frac{16}{50}, c_{3}=\frac{9}{50}$ and therefore the density distribution functions become

$$
f_{i}(x)=1+2 c_{i} \cdot \cos (2 \pi x)
$$

hence explicitly

$$
\begin{aligned}
& f_{1}(x)=1+\frac{1}{5} \cdot \cos (2 \pi x) \\
& f_{2}(x)=1-\frac{16}{25} \cdot \cos (2 \pi x) \\
& f_{3}(x)=1+\frac{9}{25} \cdot \cos (2 \pi x) .
\end{aligned}
$$

We see in this case that all functions $f_{i}$ take only positive values. Now we have all ingredients to construct the sequence $\left(\beta_{k}, \gamma_{k}\right)_{k \in \mathbb{N}}$ explicitly. We define three subsequences $\left(f_{1 k}\right)_{k \in \mathbb{N}},\left(f_{2 k}\right)_{k \in \mathbb{N}},\left(f_{3 k}\right)_{k \in \mathbb{N}}$ which approximate the distribution functions $f_{i}$ and define for $k \in \mathbb{N}$

$$
\beta_{k}:=\left\{\begin{array}{lll}
1 & \text { if } k \equiv 0 & \bmod 3 \\
2 & \text { if } k \equiv 1 & \bmod 3 \\
3 & \text { if } k \equiv 2 & \bmod 3
\end{array}\right.
$$

The elements of the imaginary parts $\gamma_{k}$ are defined as follows for $k=0,1,2, \ldots$ :

$$
\begin{aligned}
\gamma_{3 k} & :=f_{1 k} \\
\gamma_{3 k+1} & :=f_{2 k} \\
\gamma_{3 k+2} & :=f_{3 k} .
\end{aligned}
$$

According to these definitions the sequence $\left\{\gamma_{k}\right\}_{k \in \mathbb{N}}$ is not uniformly distributed modulo one since its density distribution function is 


$$
\frac{1}{3} \sum_{i=1}^{3} f_{i}(x)=1-\frac{2}{75} \cdot \cos (2 \pi x) \not \equiv 1 .
$$

It remains to explicitly construct the approximation sequences $\left(f_{i k}\right)_{k \in \mathbb{N}}$. All three sequences are defined in the same manner. For each $N \geq 1$ with $N \in \mathbb{N}$ we take rational approximations $\frac{j_{0}}{M_{N}}, \ldots, \frac{j_{N-1}}{M_{N}}$ to $f_{i}\left(\frac{0}{N}\right), f_{i}\left(\frac{1}{N}\right) \ldots, f_{i}\left(\frac{N-1}{N}\right)$ with $j_{i} \in \mathbb{Z} \geq 0$ for each $i$ and $M_{N} \in \mathbb{N}$ a common denominator. Let $s_{N}$ denote a block of length $T_{N}:=j_{0}+\ldots+j_{N-1}$ that has $\frac{t}{N}$ appearing $j_{t}$ times. Define the sequence $\left(f_{i k}\right)_{k \in \mathbb{N}}$ by $2^{2^{1}+T_{1}}$ consecutive $s_{1}$ 's followed by $2^{2^{2}+T_{2}}$ consecutive $s_{2}$ 's followed by $2^{2^{3}+T_{3}}$ consecutive $s_{3}$ 's and so on. Then, if $N$ is large enough, this yields for all $m \in \mathbb{Z}\{0\}$ :

$$
\frac{1}{N} \sum_{k=1}^{N} e^{2 \pi i \gamma_{k} m} \approx \frac{1}{N} \sum_{t=0}^{N-1} f_{i}\left(\frac{t}{N}\right) e^{2 \pi i \frac{t}{N} m} \approx \int_{0}^{1} f_{i}(x) e^{2 \pi i x m} d x=\widehat{f}_{i}(m) .
$$

Hence, for all $\epsilon_{1}>0$ we find an $N_{i} \in \mathbb{N}$ such that for all $N \geq N_{i}$

$$
\left|\frac{1}{N} \sum_{t=0}^{N-1} f_{i}\left(\frac{t}{N}\right) e^{2 \pi i \frac{t}{N} m}-\widehat{f}_{i}(m)\right|<\epsilon_{1} .
$$

Taking $N_{\max }=\max \left\{N_{1}, N_{2}, N_{3}\right\}$ we see that (2.13) is valid. Thus the sequence $\left(\gamma_{k}\right)_{k \in \mathbb{N}}$ is not uniformly distributed modulo one although (2.8) holds for the two dimensional sequence $\left(\beta_{k}, \gamma_{k}\right)_{k \in \mathbb{N}}$.

From the construction above it is clear that there are several possibilities to obtain similar sequences with different parameters chosen in (2.15). Considering more than three different weights it is also straightforward that such counterexamples exist, since in this case we add even more degrees of freedom to the linear system in $(2.14)$. 


\title{
Theory of Epstein zeta-functions
}

\author{
"Die ganze Theorie der Formen vom zweiten Grade, mit zwei \\ Variablen, kann nämlich als Theorie der complexen Zahlen von der \\ Form $x+y \sqrt{D}$ aufgefasst werden."
}

Eduard Kummer (1810-1893)

In this chapter we explore the theory of Epstein zeta-functions, mainly those associated with binary quadratic forms. For this purpose we introduce the concept of equivalent forms, the discriminant and a principal form. The class number will be a central quantity to classify quadratic forms. We show the intimate connection to quadratic number fields and the concepts of ideals, unique factorization and the class group. This leads the way to generating functions like Epstein and Dedekind zeta-functions. We explore further representations of general Epstein zeta-functions via Hecke L-functions. In the end of the chapter we consider Epstein zeta-functions associated with quadratic forms of more than two variables. We review recent results about the location of nontrivial zeros, in particular on the existence of zeros off the critical line.

\subsection{Binary quadratic forms}

\subsubsection{Basic definitions}

We define a quadratic form as a homogeneous quadratic polynomial $q$ in several but at least two variables, i.e. $q \in k[\mathbf{x}]$ with $\lambda^{2} q(\mathbf{x})=q(\lambda \mathbf{x})$ for all $\mathbf{x} \in k^{n}$, where $\mathbf{x}=\left(X_{1}, \ldots, X_{n}\right)$ and $k$ is a field or a commutative ring.

It is convenient to rewrite quadratic forms as

$$
q=\sum_{1 \leq i, j \leq n} a_{i j} X_{i} X_{j}=\mathbf{x}^{\mathrm{t}} \mathcal{Q} \mathbf{x}
$$


where $\mathcal{Q}=\left(a_{i j}\right)$ is a symmetric matrix. As mentioned above, we are specifically interested in quadratic forms of two variables, $X, Y$ in place of $X_{1}, X_{2}$. Those so-called binary quadratic forms are given by

$$
q=a X^{2}+b X Y+c Y^{2}
$$

or in matrix form via

$$
\mathcal{Q}:=\left(\begin{array}{cc}
a & \frac{b}{2} \\
\frac{b}{2} & c
\end{array}\right) .
$$

Here $a, b, c$ are integers in the sequel and we can therefore denote $q$ for short also by $(a, b, c)$.

Two binary quadratic forms $q$ and $q^{\prime}$ with integer coefficients are called weakly equivalent, if the associated symmetric matrices $\mathcal{Q}$ and $\mathcal{Q}^{\prime}$ are similar, that means

$$
\mathcal{Q}^{\prime}=\mathcal{P}^{\mathrm{t}} \mathcal{Q} \mathcal{P} \quad \text { for some } \quad \mathcal{P} \in G L_{2}(\mathbb{Z}) .
$$

If $\mathcal{P} \in S L_{2}(\mathbb{Z})$, then $q$ and $q^{\prime}$ are said to be equivalent which we shall denote by

$$
q \sim q^{\prime}
$$

We recall that the general linear group above is defined to be the set of all $2 \times 2$ matrices with integral entries and determinant equal to \pm 1 in order to have an inverse with integral entries, hence

$$
G L_{2}(\mathbb{Z})=\left\{\left(\begin{array}{ll}
r & s \\
t & u
\end{array}\right) \in \mathbb{Z}^{2 \times 2}: r u-s t= \pm 1\right\} .
$$

The special linear group $S L_{2}(\mathbb{Z})$ is the subgroup of matrices with determinant 1 . It is straightforward that both weak equivalence and equivalence are indeed equivalence relations.

The following classical result due to Lagrange shows why equivalence is an important notion in the context of quadratic forms.

Theorem 3.1. Equivalent binary quadratic forms represent the same integers.

Proof: Assuming that $m=\mathbf{x}^{\mathrm{t}} \mathcal{Q}^{\prime} \mathbf{x}$ for some $\mathbf{x} \in \mathbb{Z}^{2}$ and $\mathcal{Q}^{\prime}=\mathcal{P}^{\mathrm{t}} \mathcal{Q} \mathcal{P}$ we get

$$
m=\mathbf{x}^{\mathrm{t}} \mathcal{P}^{\mathrm{t}} \mathcal{Q} \mathcal{P} \mathbf{x}=\mathbf{y}^{\mathrm{t}} \mathcal{Q} \mathbf{y}
$$

with $\mathbf{y}=\mathcal{P} \mathbf{x} \in \mathbb{Z}^{2}$. Obviously the converse is true by symmetry.

In order to classify the equivalence classes we define the discriminant of $q=(a, b, c)$ by 


$$
D:=b^{2}-4 a c
$$

which equals $-4 \cdot \operatorname{det}\left(\begin{array}{ll}a & \frac{b}{2} \\ \frac{b}{2} & c\end{array}\right)$ but of course the factor -4 doesn't carry any information about $q$. Equivalent forms have the same discriminant whereas the converse is not true in general.

In the following we shall assume without loss of generality that $a>0$ since $(a, b, c) \sim(-a,-b,-c)$. We can now represent a binary quadratic form in terms of its discriminant as

$$
a X^{2}+b X Y+c Y^{2}=a(X-\xi Y)(X-\bar{\xi} Y)
$$

with

$$
\xi=\frac{-b+\sqrt{D}}{2 a}, \quad \bar{\xi}=\frac{-b-\sqrt{D}}{2 a} .
$$

That means the form $(a, b, c)$ splits into a product of two rational linear forms if, and only if, the discriminant $D$ is a square. If $D<0$ and $a>0$, then

$$
4 a \cdot q(\mathbf{x})=4 a^{2} X^{2}+4 a b X Y+4 a c Y^{2}=(2 a X+b Y)^{2}-D Y^{2} \geq 0 .
$$

So $q$ takes only non-negative values and does not vanish except for $\mathbf{x}=\mathbf{0}$, so in this case $q$ is said to be positive definite. However, if $D>0$, then

$$
q\left(\begin{array}{l}
1 \\
0
\end{array}\right)=a>0>-a D=q\left(\begin{array}{c}
b \\
-2 a
\end{array}\right) .
$$

In this case $q$ takes both, positive and negative values, and $q$ is said to be indefinite. In the sequel we shall assume that $D$ is not a square and satisfies $D \equiv 0$ or $1 \bmod 4$. Any such integer is called a fundamental discriminant.

\subsubsection{Class number}

For each fundamental discriminant there exists indeed at least one equivalence class, represented by the so-called principal form

$$
\begin{aligned}
\left(1,0, \frac{-D}{4}\right) & =X^{2}-\frac{1}{4} D Y^{2} & & \text { if } D \equiv 0 \bmod 4 \\
\left(1,0,-\frac{D-1}{4}\right) & =X^{2}+X Y-\frac{1}{4}(D-1) Y^{2} & & \text { if } D \equiv 1 \quad \bmod 4 .
\end{aligned}
$$

Given a fundamental discriminant $D$, the number $h(D)$ of equivalence classes with discriminant $D$ is said to be the class number of $D$. Looking at (3.3) we surely have $h(D) \geq 1$. In order to answer the question how large $h(D)$ can be we follow again Lagrange. 
Theorem 3.2. Every equivalence class to a fundamental discriminant $D=b^{2}-4 a c$ contains at least one form $(a, b, c)$ satisfying

$$
|b| \leq|a| \leq|c| .
$$

We omit the proof but note as an example, that for the form $(5,4,1)$ we can find the equivalent principal form $(1,0,1)$. From Theorem 3.2 one can as well show that the class number is finite, i.e.

$$
h(D)<\infty .
$$

In particular by leveraging Theorem 3.2 again, one can derive an explicit though poor bound for the class number in case of positive definite forms, i.e. $D<0$. It follows that

$$
h(D) \leq \frac{2+\sqrt{3}}{3}|D| .
$$

For example if we look at $D=-4$ we obtain with this bound $h(-4) \leq 4.976 \ldots$ However using Theorem 3.2 directly we get

$$
-D=4 a c-b^{2} \geq 4 a^{2}-b^{2} \geq 3 a^{2},
$$

therefore

$$
|b| \leq|a| \leq \sqrt{\frac{-D}{3}}
$$

hence for $D=-4$ we obtain

$$
|b| \leq|a| \leq 1
$$

In view of $-4=D=b^{2}-4 a c$ it follows that $b$ is even, hence $b=0$ and $a=c=1$. So we have

$$
h(-4)=1,
$$

and every form with discriminant -4 is equivalent to the principal form $(1,0,1)$. As a nice number-theoretical application this can be used to show the Two-Square Theorem, i.e. that every prime $p \equiv 1 \bmod 4$ can be written as a sum of two integer squares. Fermat proved this with his method of infinite descent, Gauss on the other hand obtained the following refinement of Theorem 3.2 for positive definite forms.

Theorem 3.3. For every form $(A, B, C)$ to a fundamental discriminant $D<0$ there exists a unique equivalent form $(a, b, c)$ satisfying either

$$
-a<b \leq a<c
$$

or

$$
0 \leq b \leq a=c,
$$

and such a form is said to be reduced. 
Next we show a few cases of small negative fundamental discriminants. For $D=-3$ we have $h(-3)=1$, the reduced form being $(1,1,1)$. The case $D=-4$ we already discussed above and for $D=-7$ we see, since $b$ has to be odd and $7 \geq 4 a c-b^{2} \geq 3 a^{2}$, that $h(-7)=1$ with the reduced form $(1,1,2)$. For $D=-15$ a more elaborated but straightforward analysis shows $h(-15)=2$, where the two reduced forms are given by $(1,1,4)$ and $(2,1,2)$. In general the class number is a rather chaotic function of $D$.

It was again Gauss who claimed that in case of negative $D$, that is for positive definite forms, the class number is one, i.e. $h(D)=1$ only for nine values, namely

$$
-D=3,4,7,8,11,19,43,67,163 .
$$

He conjectured moreover that, as $-D \rightarrow \infty$,

$$
h(D) \rightarrow \infty
$$

The latter conjecture was proved by Heilbronn in 1934, whereas the first claim was shown by Baker and independently by Stark 1966/67. An essentially correct proof by the amateur Heegener from 1952 had been ignored by the community for long.

\subsubsection{Connection to quadratic number fields}

In 1847 Kummer was the first to note that the theory of binary quadratic forms can be translated into the language of quadratic number fields.

A quadratic number field is a subfield $\mathbb{K}$ of $\mathbb{C}$ which has, considered as a $\mathbb{Q}$-vector space, dimension two. In this case, for $\alpha \in \mathbb{K} \backslash \mathbb{Q}$, there exist rationals $r, s$ such that

$$
\alpha^{2}=r+s \cdot \alpha
$$

respectively

$$
a \alpha^{2}+b \alpha+c=0
$$

or

$$
\alpha=\frac{-b}{2 a} \pm \frac{1}{2 a} \sqrt{D}
$$

with $D:=b^{2}-4 a c$ where $a \in \mathbb{N}$ and $b, c \in \mathbb{Z}$ are coprime. We denote by $\mathbb{Q}[\sqrt{d}]$ all linear combinations $\mathbb{Q}+\mathbb{Q} \sqrt{d}$. Writing $D=m^{2} d$ with squarefree $d$, this shows that $\alpha \in \mathbb{Q}+\mathbb{Q} \sqrt{d}$, i.e. $\mathbb{K} \subset \mathbb{Q}[\sqrt{d}]$. Since also $\mathbb{Q}+\mathbb{Q} \sqrt{d} \subset \mathbb{Q}+\mathbb{Q} \alpha=\mathbb{K}$, it follows that

$$
\mathbb{K}=\mathbb{Q}+\mathbb{Q} \sqrt{d}=\mathbb{Q}[\sqrt{d}]
$$


Higher powers of $\sqrt{d}$ can be written as rational linear combinations of 1 and $\sqrt{d}$. It is straightforward to verify the field axioms, for example, given $a+b \sqrt{d} \neq 0$,

$$
\frac{1}{a+b \sqrt{d}}=\frac{1}{a+b \sqrt{d}} \cdot \frac{a-b \sqrt{d}}{a-b \sqrt{d}}=\frac{a-b \sqrt{d}}{a^{2}-d b^{2}}
$$

is the multiplicative inverse in $\mathbb{Q}[\sqrt{d}]$, here $a^{2}-d b^{2}$ does not vanish since $\sqrt{d}$ is irrational and $\operatorname{dim}_{\mathbb{Q}} \mathbb{K}=2$.

This describes already all quadratic number fields, however, we prefer to denote them by $\mathbb{Q}(\sqrt{d})$ as the smallest subfield of $\mathbb{C}$ that contains $\mathbb{Q}$ and $\sqrt{d}$. For squarefree $d$ this definition coincides with $\mathbb{Q}[\sqrt{d}]$ but we want to distinguish from polynomial rings in general.

The concept of algebraic integers as analogue for the rational integers in algebraic extensions of $\mathbb{Q}$ has been established by Dedekind in the 1870's generalizing work of Gauss, Dirichlet and others.

A complex number $\alpha$ is said to be an algebraic number if it is a root of a monic polynomial with rational coefficients. If there is a monic polynomial with root $\alpha$ and integer coefficients, $\alpha$ is an algebraic integer, i.e.

$$
P(\alpha)=0 \text { for } P=X^{d}+a_{d-1} X^{d-1}+\ldots+a_{1} X+a_{0}
$$

with $a_{d-1}, \ldots, a_{1}, a_{0} \in \mathbb{Z}$. In the case of quadratic number fields it suffices to consider quadratic polynomials thanks to Gauss' lemma, that means

$$
P=(X-\alpha)\left(X-\alpha^{\prime}\right)=X^{2}-\left(\alpha+\alpha^{\prime}\right) X+\alpha \alpha^{\prime},
$$

where $\alpha^{\prime}$ is the conjugate root of $\alpha$. For $\alpha$ being an algebraic integer it is therefore needed that both the trace

$$
\operatorname{tr}(\alpha):=\alpha+\alpha^{\prime}\left(=\operatorname{tr}\left(\alpha^{\prime}\right)\right)
$$

and the norm

$$
N(\alpha):=\alpha \cdot \alpha^{\prime}\left(=N\left(\alpha^{\prime}\right)\right)
$$

are rational integers. First examples of algebraic integers are $i=\sqrt{-1}$ for the monic polynomial $X^{2}+1=(X-i)(X+i)$ and $\varrho=\frac{1}{2}(1+\sqrt{-3})$ for the polynomial $(X-\varrho)\left(X-\varrho^{\prime}\right)$ respectively. 
Theorem 3.4. The set $\mathcal{O}_{d}$ of algebraic integers in the quadratic number field $\mathbb{Q}(\sqrt{d})$ has the structure of a commutative ring and is called the ring of algebraic integers in $\mathbb{Q}(\sqrt{d})$. Moreover,

$$
\mathcal{O}_{d}=\mathbb{Z}[\vartheta]
$$

where

$$
\vartheta= \begin{cases}\frac{1}{2}(1+\sqrt{d}) & \text { if } d \equiv 1 \bmod 4 \\ \sqrt{d} & \text { otherwise. }\end{cases}
$$

Here $d$ is supposed to be a squarefree integer, hence $d \not \equiv 0 \bmod 4$. According to this theorem we have the rings $\mathcal{O}_{-1}=\mathbb{Z}[i]$ and $\mathcal{O}_{-3}=\mathbb{Z}\left[\frac{1}{2}(1+\sqrt{-3})\right]$ of Gaussian and Eisenstein integers, respectively, corresponding to the examples of algebraic integers above.

Elements of norm \pm 1 are called units, they are determined by the equation

$$
A^{2}-d B^{2}= \pm 1
$$

if $d$ is congruent to $2,3 \bmod 4$ or

$$
A^{2}-d B^{2}= \pm 4
$$

if $d$ is congruent to $1 \bmod 4$.

If $d>0$, then $\mathbb{Q}(\sqrt{d}) \subset \mathbb{R}$ and $\mathbb{Q}(\sqrt{d})$ is said to be a real quadratic number field and in that case there exist infinitely many units, which can be found by solving the corresponding Pell equation. Otherwise, if $d<0$, then $\mathbb{Q}(\sqrt{d})$ is an imaginary quadratic number field and there are only finitely many units, namely

- $\pm 1, \pm i$ for $d=-1$,

- $\pm 1 \pm \omega \pm \omega^{2}$ with $\omega=\exp \left(\frac{2 \pi i}{6}\right)$ for $d=-3$,

- \pm 1 in all other cases of negative $d$.

In general there is no unique factorization into irreducible elements in rings of integers, for example,

$$
6=2 \cdot 3=(1+\sqrt{-5})(1-\sqrt{-5})
$$

are two different factorizations into products of algebraic integers in $\mathcal{O}_{-5}$. Here all factors are irreducible which means that in every further factorization there appears a unit.

In order to overcome this difficulty in the arithmetic of quadratic number fields, Dedekind introduced the notion of an 'ideal' in the 1870's.

Given a commutative ring $\mathcal{R}$, a subset $\mathcal{I} \subset \mathcal{R}$ is called an ideal of $\mathcal{R}$, if 
- $\mathcal{I}$ is an additive subgroup of $\mathcal{R}$,

- $\mathcal{I}$ is multiplicatively closed with respect to multiplication with ring elements, i.e., $r i \in \mathcal{I}$ for all $r \in \mathcal{R}, i \in \mathcal{I}$.

In the following we shall consider ideals in the ring $\mathcal{R}=\mathcal{O}_{d}$ of integers of some quadratic number field $\mathbb{Q}(\sqrt{d})$.

If $\alpha_{1}, \ldots, \alpha_{m}$ are integers, then

$$
\mathcal{I}=\alpha_{1} \mathcal{O}_{d}+\ldots+\alpha_{m} \mathcal{O}_{d}
$$

is an ideal of $\mathcal{O}_{d}$, which we denote by $\left[\alpha_{1}, \ldots, \alpha_{m}\right]$ and we say that $\mathcal{I}$ is generated by $\alpha_{1}, \ldots, \alpha_{m}$. Indeed every ideal of $\mathcal{O}_{d}$ is finitely generated, hence of this form.

An ideal $[\alpha]$ generated by a single integer is called a principal ideal, for example $[1]=\mathcal{O}_{d}$, or

$$
[1+i]=(1+i) \mathcal{O}_{-1}=\{a+b i \in \mathbb{Z}[i]: a \equiv b \bmod 2\} .
$$

However as seen in $(3.4),[2,1+\sqrt{-5}]$ cannot be a principal ideal of $\mathcal{O}_{-5}$. Given ideals $\mathfrak{a}=\left[\alpha_{1}, \ldots, \alpha_{m}\right]$ and $\mathfrak{b}=\left[\beta_{1}, \ldots, \beta_{n}\right]$, both of some $\mathcal{O}_{d}$, their product is defined by

$$
\mathfrak{a} \mathfrak{b}=\left[\ldots, \alpha_{i} \beta_{j}, \ldots\right] .
$$

This definition is independent of the choice of basis elements $\alpha_{i}, \beta_{j}$. Then, if $\mathfrak{c}=\mathfrak{a} \mathfrak{b}$, we say $\mathfrak{a}$ divides $\mathfrak{c}$ or $\mathfrak{a}$ is a divisor of $\mathfrak{c}$. This is denoted by $\mathfrak{a} \mid \mathfrak{c}$ although it means $\mathfrak{c} \subset \mathfrak{a}$. Now divisibility of ideals is quite similar to its counterpart for integers.

Theorem 3.5. Every ideal has finitely many distinct divisors.

This allows to prove unique factorization of ideals. For this aim we call an ideal a prime ideal if its only divisors are the whole $\operatorname{ring} \mathcal{O}_{d}$ and the ideal itself. Then, given any two ideals $\mathfrak{a}, \mathfrak{b}$ of $\mathcal{O}_{d}$, there exists a unique ideal $\mathfrak{c}$ such that

- $\quad \mathfrak{c} \mid \mathfrak{a}$ and $\mathfrak{c} \mid \mathfrak{b}$,

- $\mathfrak{d} \mid \mathfrak{a}$ and $\mathfrak{d}|\mathfrak{b}, \quad \Rightarrow \quad \mathfrak{d}| \mathfrak{c}$.

Hence, this ideal $\mathfrak{c}$ is called the greatest common divisor of $\mathfrak{a}$ and $\mathfrak{b}$, similar to the classical arithmetic of the rational integers. Moreover, if $\mathfrak{p}$ is a prime ideal satisfying $\mathfrak{p} \mid \mathfrak{a} \mathfrak{b}$, then $\mathfrak{p} \mid \mathfrak{a}$ or $\mathfrak{p} \mid \mathfrak{b}$. This is essentially the 'Lemma of Euclid' and follows by the same reasoning as for the rational primes. With this we get to the fundamental theorem for ideals.

Theorem 3.6. Every ideal $\mathfrak{a} \subsetneq \mathcal{O}_{d}$ can be factorized into a product of prime ideals which is unique up to the ordering of the factors. 
As an example in $\mathbb{Z}[i]$ we have

$$
[15]=[3][2+i][2-i]
$$

since $15=3 \cdot 5=3 \cdot(2+i)(2-i)$.

Now we describe the prime ideals in more detail. For this purpose we simplify the notation $\mathfrak{a} \mid[n]$ by writing $\mathfrak{a} \mid n$ and saying that $\mathfrak{a}$ divides $n$ which means $n \subset \mathfrak{a}$. Moreover, we generalize modular arithmetic by writing

$$
\alpha \equiv \beta \bmod \mathfrak{a}
$$

if $\mathfrak{a} \mid(\alpha-\beta)$. This notion of congruence modulo an ideal $\mathfrak{a}$ implies an equivalence relation on $\mathcal{O}_{d}$ and the equivalence classes are the residue classes modulo $\mathfrak{a}$. Their number $N(\mathfrak{a})$ is finite and called the norm of $\mathfrak{a}$.

By division with remainder every ideal $\mathfrak{a}$ of $\mathcal{O}_{d}=\mathbb{Z}+\mathbb{Z} \vartheta$ with $\vartheta$ according to Theorem 3.4 is of the form

$$
\mathfrak{a}=a \mathbb{Z}+(b+c \vartheta) \mathbb{Z}=[a, b+c \vartheta]
$$

providing an 'integral' basis for this module or lattice in the ring $\mathcal{O}_{d}$ of integers. Hence, there are

$$
N(\mathfrak{a})=|a c|=\left|\operatorname{det}\left(\begin{array}{ll}
a & 0 \\
b & c
\end{array}\right)\right|
$$

residue classes $\bmod \mathfrak{a}$. In general, if $\alpha_{1}, \ldots, \alpha_{n}$ is any basis of an ideal $\mathfrak{a}$ of some ring $\mathcal{R}=\left[\omega_{1}, \ldots, \omega_{n}\right]$ and $\alpha_{i}=\sum_{1 \leq j \leq n} a_{i j} \omega_{j}$ for $i=1, \ldots, n$, then

$$
N(\mathfrak{a})=\left|\operatorname{det}\left(a_{i j}\right)\right| .
$$

The norm is multiplicative, i.e. $N(\mathfrak{a} \mathfrak{b})=N(\mathfrak{a}) N(\mathfrak{b})$. In order to determine the unique prime ideal factorization the following statement is crucial.

Theorem 3.7. Every prime ideal $\mathfrak{p}$ must divide a rational prime number $p$. Moreover, $p$ is the least rational positive integer in $\mathfrak{p}$, hence $p$ is unique.

For a rational prime $p$, let

$$
[p]=\mathfrak{p}_{1} \cdot \ldots \cdot \mathfrak{p}_{e}
$$

be the prime ideal factorization. Taking the norm, we get

$$
p^{n}=N([\mathfrak{p}])=N\left(\mathfrak{p}_{1}\right) \cdot \ldots \cdot N\left(\mathfrak{p}_{e}\right),
$$

where $n=\operatorname{dim}_{\mathbb{Q}} \mathbb{K}$ is the degree of the underlying number field $\mathbb{K}$, so $n=2$ in the case of a quadratic number field $\mathbb{K}=\mathbb{Q}(\sqrt{d})$. It thus follows that the norm of a prime ideal must be a prime power, say $N([\mathfrak{p}])=p^{f}$, where $f$ is 
called the degree of $\mathfrak{p}$.

Let $(\dot{\bar{p}})$ be the Kronecker symbol, for a precise definition see for instance [12], which is equal to the Legendre symbol in case of odd $p$, and define

$$
\Delta:=\left\{\begin{array}{lll}
d & \text { if } \quad d \equiv 1 \quad \bmod 4 \\
4 d & \text { if } \quad d \equiv 2,3 \quad \bmod 4
\end{array}\right.
$$

to be the discriminant of $\mathbb{K}=\mathbb{Q}(\sqrt{d})$. We then can state the following important theorem about the splitting of rational primes in quadratic number fields.

Theorem 3.8. For a rational prime $p$ and a quadratic number field $\mathbb{K}=$ $\mathbb{Q}(\sqrt{d})$ we have

$$
[p]=\left\{\begin{array}{lll}
\mathfrak{p} & & \\
\mathfrak{p q} & \text { with } & \mathfrak{p} \neq \mathfrak{q}, N(\mathfrak{p})=N(\mathfrak{q})=p \\
\mathfrak{p}^{2} & \text { with } & N(\mathfrak{p})=p
\end{array}\right.
$$

if, and only if

$$
\left(\frac{\Delta}{p}\right)=\left\{\begin{array}{l}
-1 \\
+1 \\
0
\end{array}\right.
$$

Next we suppose that $\mathfrak{a}$ and $\mathfrak{b}$ are two ideals and that there exist two principal ideals $[\alpha]$ and $[\beta]$ such that

$$
[\alpha] \mathfrak{a}=\mathfrak{b}[\beta] .
$$

Then we say that $\mathfrak{a}$ and $\mathfrak{b}$ belong to the same ideal class and we write $\mathfrak{a} \sim \mathfrak{b}$. This definition implies an equivalence relation on the set of ideals and the ideal classes are the equivalence classes. We deduce further

- $\mathfrak{a} \sim \mathcal{O}_{d} \quad \Leftrightarrow \quad \mathfrak{a}$ is principal

- $\mathfrak{a} \sim \mathfrak{b}, \quad \mathfrak{c} \sim \mathfrak{d} \Rightarrow \mathfrak{a} \mathfrak{c} \sim \mathfrak{b} \mathfrak{d}$

- $\mathfrak{a} \mathfrak{a} \sim \mathfrak{b} \mathfrak{c} \quad \Rightarrow \quad \mathfrak{a} \sim \mathfrak{b}$

Hence, the ideals of $\mathcal{O}_{d}$ can be partitioned into equivalence classes, the socalled ideal-classes. By showing that every ideal class contains an ideal $\mathfrak{b}$ satisfying $N(\mathfrak{b}) \leq M$ with some absolute constant $M$ one can prove the following.

Theorem 3.9. The number of ideal classes is finite. 
This reminds us of the finiteness of the class number and both sets of equivalence classes are indeed closely related. To see that we consider a ring of integers $\mathcal{O}_{d}$ of a quadratic number field $\mathbb{Q}(\sqrt{d})$. Let $\mathfrak{a}=\left[\alpha_{1}, \alpha_{2}\right]$ be an ideal of $\mathcal{O}_{d}$ with basis elements $\alpha_{1}, \alpha_{2}$ and their conjugates $\alpha_{1}^{\prime}, \alpha_{2}^{\prime}$ satisfying

$$
\alpha_{1} \alpha_{2}^{\prime}-\alpha_{1}^{\prime} \alpha_{2}=N(\mathfrak{a}) \cdot \sqrt{\Delta}
$$

where $\Delta$ is the discriminant as defined above. Corresponding to $\mathfrak{a}$ we can now define a quadratic form $(a, b, c)$ by setting

$$
a X^{2}+b X Y+c Y^{2}=\frac{N\left(\alpha_{1} X+\alpha_{2} Y\right)}{N(\mathfrak{a})}=\frac{1}{N(\mathfrak{a})}\left(\alpha_{1} X+\alpha_{2} Y\right)\left(\alpha_{1}^{\prime} X+\alpha_{2}^{\prime} Y\right)
$$

It follows quickly that then $a, b, c$ are integers since $a=\frac{N\left(\alpha_{1}\right)}{N(\mathfrak{a})}$ and so forth, and the discriminant of $(a, b, c)$ is given by

$$
b^{2}-4 a c=\frac{1}{N(\mathfrak{a})^{2}}\left(\alpha_{1} \alpha_{2}^{\prime}-\alpha_{1}^{\prime} \alpha_{2}\right)^{2}=\Delta,
$$

which is equal to $d$ if $d \equiv 1 \bmod 4$, or equal to $4 d$ otherwise. If $\Delta<0$, the quadratic field $\mathbb{Q}(\sqrt{d})$ is imaginary so that $a>0$ and $(a, b, c)$ is positive definite. Varying the basis yields equivalent forms.

Theorem 3.10. Every positive definite quadratic form $(a, b, c)$ of discriminant $\Delta$ belongs to an ideal $\mathfrak{a}$ with basis $\alpha_{1}, \alpha_{2}$.

This holds since it is not difficult to show that $a, \frac{1}{2}(b-\sqrt{\Delta})$ form a basis for the ideal $\mathfrak{a}=\left[a, \frac{1}{2}(b-\sqrt{\Delta})\right]$.

We can deduce that equivalent quadratic forms belong to equivalent ideals and conversely. The corresponding number of classes are therefore equal to the class number $h(D)$ as introduced for forms. Dirichlet achieved with his analytic class number formula a closed expression for this quantity. Another way of computing $h(D)$ is by the reduction Theorems 3.2 and 3.3 of Lagrange and Gauss above.

Now given a ring of integers $\mathcal{O}_{d}$, we denote by $\mathcal{I}_{d}$ the set of all ideals and by $\mathcal{P}_{d}$ the subset of all principal ideals. Unique factorization in $\mathcal{O}_{d}$ is then equivalent to $\mathcal{P}_{d}=\mathcal{I}_{d}$ but this doesn't hold often. In order to quantify the extent to which it fails we consider fractional ideals.

A fractional ideal of $\mathcal{O}_{d}$ is a subset of the underlying field $\mathbb{Q}(\sqrt{d})$ which is of the form $\frac{1}{\gamma} \mathfrak{c}$, where $\mathfrak{c}$ is an ideal of $\mathcal{O}_{d}$ and $\gamma$ is a non-zero element of $\mathcal{O}_{d}$, such an ideal is said to be principal if $\mathfrak{c}$ is principal.

Then the set of fractional ideals forms an abelian group and the inverse $\mathfrak{a}^{-1}$ of an ideal $\mathfrak{a}$ of $\mathcal{O}_{d}$ is given by 


$$
\mathfrak{a}^{-1}=\left\{\alpha \in \mathbb{Q}(\sqrt{d}): \alpha \mathfrak{a} \subset \mathcal{O}_{d}\right\} .
$$

Moreover, writing $\mathcal{F}_{d}$ for this set/group of fractional ideals and $\mathcal{P} \mathcal{F}_{d}$ for its subset/subgroup of principal fractional ideals, the quotient

$$
\mathfrak{c}_{d}=\mathcal{F}_{d} / \mathcal{P} \mathcal{F}_{d}
$$

is again a group and called the class group of $\mathbb{Q}(\sqrt{d})$ respectively $\mathcal{O}_{d}$. Its elements are given by the different ideal classes corresponding to the equivalence of ideals $\sim$ defined above.

Hence, the order of the class group is the class number $h(D)$, and its size measures the deviation from unique factorization in $\mathcal{O}_{d}$.

In fact, if $\mathfrak{c}_{d}$ is trivial, then $\mathcal{F}_{d}=\mathcal{P} \mathcal{F}_{d}$ and intersecting with the set of genuine ideals of $\mathcal{O}_{d}$ leads to $\mathcal{I}_{d}=\mathcal{P}_{d}$ which implies unique factorization. In any case we know at least that

$$
\# \mathfrak{c}_{d}=h(d)<\infty
$$

\subsection{Generating functions associated with forms and ideals}

Now we consider generating functions associated with forms and ideals. For forms building on the fundament described above we recall definition (1.1) of the Epstein zeta-function given for a positive definite form with symmetric matrix $\mathcal{Q}$ by

$$
\zeta(s ; \mathcal{Q})=\sum_{0 \neq \mathbf{x} \in \mathbb{Z}^{n}} \mathbf{x}^{\mathrm{t}} \mathcal{Q} \mathbf{x}^{-s} .
$$

On the other hand given a number field $k=\mathbb{Q}(\sqrt{d})$ the Dedekind zetafunction is defined by

$$
\zeta_{k}(s):=\sum_{\mathfrak{a}} N(\mathfrak{a})^{-s}=\prod_{\mathfrak{p}}\left(1-N(\mathfrak{p})^{-s}\right)^{-1}
$$

where the summation is over all ideals $\mathfrak{a} \neq\{0\}$ of $\mathcal{O}_{d}$ and the product runs over all prime ideals. The identity between the Dirichlet series and the Euler product follows from unique prime ideal factorization, i.e. Theorem 3.6 in just the same way as in the case of the Riemann zeta-function

$$
\zeta(s):=\sum_{n \geq 1} n^{-s}=\prod_{p}\left(1-p^{-s}\right)^{-1},
$$

which can be considered as the Dedekind zeta-function associated with the field $\mathbb{Q}$ of rational numbers and unique prime number factorization. 
Case $k=\mathbb{Q}(i)$ : As the most straight forward example we have the case of $k=\mathbb{Q}(i)$ with $i=\sqrt{-1}$, the Gaussian field. The discriminant $\Delta$ of this field is -4 . Since $h(-4)=1$, the ring of integers $\mathcal{O}_{-1}=\mathbb{Z}[i]$ is a unique factorization domain and all ideals are principal ideals. In view of the splitting of primes according to Theorem 3.8 it follows that

$$
\begin{aligned}
\zeta_{k}(s) & =\prod_{\mathfrak{p}}\left(1-N(\mathfrak{p})^{-s}\right)^{-1} \\
& =\prod_{p \mid \Delta}\left(1-p^{-s}\right)^{-1} \cdot \prod_{p:\left(\frac{\Delta}{p}\right)=+1}\left(1-p^{-s}\right)^{-2} \cdot \prod_{p:\left(\frac{\Delta}{p}\right)=-1}\left(1-p^{-s}\right)^{-1} \\
& =\zeta(s) \cdot L\left(s ; \chi_{\Delta}\right)
\end{aligned}
$$

where $n \mapsto \chi_{\Delta}(n):=\left(\frac{n}{\Delta}\right)$ is the Kronecker symbol defined in the previous section and

$$
L\left(s ; \chi_{\Delta}\right):=\prod_{p}\left(1-\chi_{\Delta}(p) p^{-s}\right)^{-1}=\sum_{n \geq 1} \chi_{\Delta}(n) n^{-s}
$$

is the Dirichlet L-function to the character $\chi_{\Delta}$. Here $\chi_{\Delta}$ is the LegendreKronecker character, see for example Narkiewicz [53] for precise definitions. The character which is identically one, is called the principal character and is denoted by $\chi_{0}$. In the special case $k=\mathbb{Q}(i)$ we have $\chi_{4}=\chi \bmod 4 \neq \chi_{0}$ $\bmod 4$ and

$$
L\left(s ; \chi_{-4}\right):=1-3^{-s}+5^{-s}-7^{-s}+9^{-s} \mp \ldots .
$$

Now every quadratic form $(a, b, c)$ of discriminant $D=-4$ is equivalent to the principal form $(1,0,1)=X^{2}+Y^{2}$ which is the norm of $x+i y$ in $\mathbb{Q}(i)$. Taking into account that $\mathbb{Z}[i]$ carries four units $+1,-1, i,-i$ we find

$$
\zeta(s ;(1,0,1))=4 \cdot \zeta_{\mathbb{Q}(i)}(s)=4 \zeta(s) L\left(s ; \chi_{-4}\right) .
$$

Hence, this Epstein zeta-function is essentially the Dedekind zeta-function of a quadratic number field.

Case $k=\mathbb{Q}(\sqrt{-5})$ : However if we take the example $k=\mathbb{Q}(\sqrt{-5})$, the ring of integers $\mathcal{O}_{-5}=\mathbb{Z}[\sqrt{-5}]$ does not have unique prime factorization. In this case we find via $\Delta=-20$ that $h(-20)=2$ and the two equivalence classes of quadratic forms are represented by the principal form

$$
q_{1}=(1,0,5)=X^{2}+5 Y^{2}
$$

and

$$
q_{2}=(2,2,3)=2 X^{2}+2 X Y+3 Y^{2} .
$$

Using quadratic reciprocity one can show that 
- $q_{1}$ represents all primes $p$ with

$$
\left(\frac{-1}{p}\right)=\left(\frac{5}{p}\right)=+1, \quad \text { i.e. } \quad p \equiv 1,9 \bmod 20,
$$

- $q_{2}$ represents all primes $p$ with

$$
\left(\frac{-1}{p}\right)=\left(\frac{5}{p}\right)=-1, \quad \text { i.e. } \quad p \equiv 3,7 \quad \bmod 20 .
$$

Thus, in this case the Epstein zeta-function cannot coincide with the corresponding Dedekind zeta-function of this field. In view of

$$
\chi_{-20}(p)=\left(\frac{-20}{p}\right)=\left(\frac{-4}{p}\right)\left(\frac{5}{p}\right)=\chi_{-4}(p) \chi_{5}(p)
$$

it follows from (3.5) that

$$
\zeta_{\mathbb{Q}(\sqrt{-5})}(s)=\zeta(s) \cdot L\left(s ; \chi_{-20}\right)
$$

with

$$
L\left(s ; \chi_{-20}\right)=1+3^{-s}+7^{-s}+9^{-s}-11^{-s}-13^{-s}-17^{-s}-19^{-s} \pm \ldots .
$$

Employing so-called Hecke characters $\psi$ of the related class group $\mathcal{C}_{-20}$, one can define a Hecke L-function $L(s ; \psi)$ such that

$$
\begin{aligned}
\zeta\left(s ; q_{1}\right) & =\sum_{(X, Y) \neq 0}\left(X^{2}+5 Y^{2}\right)^{-s} \\
& =2+2 \cdot 4^{-s}+2 \cdot 5^{-s}+2 \cdot 6^{-s} \ldots \\
& =\zeta_{\mathbb{Q}(\sqrt{-5})}(s)+L(s ; \psi)
\end{aligned}
$$

and

$$
\begin{aligned}
\zeta\left(s ; q_{2}\right) & =\sum_{(X, Y) \neq 0}\left(2 X^{2}+2 X Y+3 Y^{2}\right)^{-s} \\
& =2 \cdot 2^{-s}+4 \cdot 3^{-s} \ldots \\
& =\zeta_{\mathbb{Q}(\sqrt{-5})}(s)-L(s ; \psi) .
\end{aligned}
$$

For exact definitions of Hecke characters and Hecke L-functions we refer again for instance to Narkiewicz [53]. Indeed this yields

$$
L(s ; \psi)=1-2^{-s}-2 \cdot 3^{-s}+4^{-s} \pm \ldots .
$$

Here the coefficients count how many integer pairs $(X, Y)$ lead to a value $n$ represented by $q_{1}$ or $q_{2}$. Since the equivalence classes of the class group or 
the equivalence classes of the two forms are disjoint and their union covers everything, we have

$$
\zeta\left(s ; q_{1}\right)+\zeta\left(s ; q_{2}\right)=2 \cdot \zeta_{\mathbb{Q}(\sqrt{-5})}(s)
$$

where the factor 2 denotes the two units in $\mathcal{O}_{-5}=\mathbb{Z}[\sqrt{-5}]$.

\subsection{Epstein zeta-functions for $n \leq 2$}

We have now developed the tools to describe Epstein zeta-functions more thoroughly. The simplest case is obviously $n=1$, where the Epstein zetafunction coincides up to a constant factor with the Riemann zeta-function, that is

$$
\zeta\left(s ; \mathbf{I}_{1}\right)=2 \zeta(2 s)
$$

with $I_{1}$ the $1 \times 1$ identity matrix. For $n=2$, i.e. binary quadratic forms, any Epstein zeta-function can be written as a linear combination of Hecke Lfunctions associated to the class group of the corresponding number field, and its class number provides the length of those linear combinations. If the class number equals one, then the associated Epstein zeta-function is a constant multiple of the Dedekind zeta-function to the related imaginary quadratic number field $\mathbb{Q}(\sqrt{-d})$ and has an Euler product representation, as seen in (3.6) for the Gaussian field. Generally, i.e. also valid for a class number larger than one, we get

$$
\zeta(s, \mathcal{Q})=\frac{\epsilon}{h} \sum_{1 \leq j \leq h} \overline{\chi_{j}(I)} L\left(s, \chi_{j}\right)
$$

where $h$ is the class number, $\epsilon$ the number of units in the ring of integers of $\mathbb{Q}(\sqrt{-d})$, and the Hecke characters $\chi_{j}$ are evaluated at the ideals $I$ generated by $a, b-\sqrt{-d} / 2 . L\left(s, \chi_{j}\right)$ is the associated Hecke $L$-function which is defined by

$$
L(s, \chi)=\sum_{\mathfrak{n}} \frac{\chi(\mathfrak{n})}{N(\mathfrak{n})^{s}}=\prod_{\mathfrak{p}}\left(1-\frac{\chi(\mathfrak{p})}{N(\mathfrak{p})^{s}}\right)^{-1} .
$$

In general the L-functions in (3.9) are not distinct. We can rewrite (3.9) by noting that according to Theorem 3.8 for each rational prime $p$, a principal ideal $[p]$ is a prime ideal $\mathfrak{p}$ or a product of two prime ideals $\mathfrak{p}_{1} \mathfrak{p}_{2}$. If $[p]=\mathfrak{p}$, then $\chi(\mathfrak{p})=1$ and

$$
\prod_{\mathfrak{p} \mid p}\left(1-\frac{\chi(\mathfrak{p})}{N(\mathfrak{p})^{s}}\right)^{-1}=\left(1-\frac{1}{p^{2 s}}\right)^{-1} .
$$


If $[p]=\mathfrak{p}_{1} \mathfrak{p}_{2}$, then $\chi\left(\mathfrak{p}_{1}\right) \chi\left(\mathfrak{p}_{2}\right)=1$. Hence, we have $\chi\left(\mathfrak{p}_{1}\right)=\overline{\chi\left(\mathfrak{p}_{2}\right)}$ and

$$
\begin{aligned}
\prod_{\mathfrak{p} \mid p}\left(1-\frac{\chi(\mathfrak{p})}{N(\mathfrak{p})^{s}}\right)^{-1} & =\left(1-\frac{\chi\left(\mathfrak{p}_{1}\right)+\chi\left(\mathfrak{p}_{2}\right)}{p^{s}}+\frac{1}{p^{2 s}}\right)^{-1} \\
& =\left(1-\frac{2 \operatorname{Re}\left(\chi\left(\mathfrak{p}_{1}\right)\right)}{p^{2 s}}+\frac{1}{p^{2 s}}\right)^{-1}
\end{aligned}
$$

Therefore $L(s, \chi)=\overline{L(s, \chi)}$. If we now let $J$ be the number of real characters plus one-half the number of complex characters, and list the characters as $\chi_{1}, \ldots, \chi_{J}$ such that $\chi_{j} \neq \chi_{k}$ and $\chi_{j} \neq \overline{\chi_{k}}$ for $j \neq k$, we can rewrite (3.9) as

$$
\zeta(s, \mathcal{Q})=\sum_{j=1}^{J} c_{j} \cdot L\left(s, \chi_{j}\right),
$$

where

$$
c_{j}=\frac{\epsilon}{h} \cdot \chi_{j}(I)
$$

for real characters $\chi_{j}$, and

$$
c_{j}=\frac{\epsilon}{h} \cdot 2 \operatorname{Re}\left(\chi_{j}(I)\right)
$$

for complex characters $\chi_{j}$. Here we note that $J>1$ if and only if $h>1$.

For the above mentioned examples $q_{1}=X^{2}+5 Y^{2}$ and $q_{2}=2 X^{2}+2 X Y+3 Y^{2}$ of two different forms in $k=\mathbb{Q}(\sqrt{-5})$ the linear combinations are

$$
\zeta\left(s ; q_{1}\right)=L\left(s, \chi_{0}\right)+L\left(s, \chi_{1}\right)
$$

and

$$
\zeta\left(s ; q_{2}\right)=L\left(s, \chi_{0}\right)-L\left(s, \chi_{1}\right)
$$

where $L\left(s, \chi_{0}\right)=\zeta_{\mathbb{Q}(\sqrt{-5})}(s)=2 \zeta(s) L\left(s, \chi_{-20}\right)$ is again the Dedekind zetafunction of this number field. $L\left(s, \chi_{1}\right)$ is the Hecke $L$-function to the nontrivial Hecke character which coincides with $L(s ; \psi)$, the series defined in (3.7).

Hence, all Epstein zeta-functions for $n \leq 2$ can be represented in the format described in this section, essentially via (3.9), or equivalently (3.10). The situation gets more complex for $n>2$, this is what we will consider now.

\subsection{Epstein zeta-functions for $n \geq 2$}

For $n>2$ the structure of an arbitrary Epstein zeta-function becomes much more difficult to describe. We follow Steuding [66], Nakamura and Pańkowski 
[52] and start with some examples. If $\mathbf{I}_{n}$ is the $n \times n$ identity matrix we obtain for $n=4$

$$
\zeta\left(s ; \mathbf{I}_{4}\right)=8\left(1-2^{2-s}\right) \zeta(s) \zeta(s-1)
$$

and for higher dimensions $n=6$ and $n=10$

$$
\begin{aligned}
\zeta\left(s ; \mathbf{I}_{6}\right) & =-4\left(\zeta(s) L\left(s-2, \chi_{-4}\right)-4 \zeta(s-2) L\left(s, \chi_{-4}\right)\right), \\
\zeta\left(s ; \mathbf{I}_{10}\right) & =\frac{4}{5}\left(\zeta(s) L\left(s-2, \chi_{-4}\right)-4^{2} \zeta(s-2) L\left(s, \chi_{-4}\right)\right)-2 \sum_{0 \neq \lambda \in \mathbb{Z}[i]} \frac{\lambda^{4}}{(\lambda \bar{\lambda})^{s}} .
\end{aligned}
$$

For $n=12$ we need more definitions. Let

$$
L(s, \Delta):=\sum_{m=1}^{\infty} \tau(m) m^{-s}
$$

be the $L$-function attached to Ramanujan's $\tau$-function, given by

$$
\Delta(q)=\sum_{m=1}^{\infty} \tau(m) q^{m}=q \sum_{m=1}^{\infty}\left(1-q^{m}\right)^{24}
$$

with $q:=e^{2 \pi i \tau}$ and $\tau$ from the upper half-plane. Then we get

$$
\zeta\left(s ; \mathbf{I}_{12}\right)=c_{1}\left(2^{6}-2^{6-s}\right) \zeta(s) \zeta(s-4)+c_{2} L(s, \sqrt{\Delta})
$$

with some constants $c_{1}$ and $c_{2}$.

Leaving the identity matrices we mention two further examples of explicitly described Epstein zeta-functions. For the matrix

$$
\mathcal{S}_{8}=\left(\begin{array}{cc}
2 \cdot \mathbf{I}_{4} & \mathcal{A} \\
-\mathcal{A} & 2 \cdot \mathbf{I}_{4}
\end{array}\right) \quad \text { with } \quad \mathcal{A}:=\left(\begin{array}{cccc}
0 & 1 & 1 & 1 \\
-1 & 0 & -1 & 1 \\
-1 & 1 & 0 & -1 \\
-1 & -1 & 1 & 0
\end{array}\right)
$$

we get

$$
\zeta\left(s ; \mathcal{S}_{8}\right)=240 \cdot 2^{-s} \zeta(s) \zeta(s-3) .
$$

Another example, as described in [10] and [40], is related to the Leech lattice of dimension $n=24$. If $\mathcal{L}_{24}$ denotes the $24 \times 24$ matrix given by

$$
\mathcal{L}_{24}=\left(\begin{array}{cc}
4 \cdot \mathbf{I}_{12} & \mathcal{B}-2 \cdot \mathbf{I}_{12} \\
\mathcal{B}^{\mathrm{t}} & 4 \cdot \mathbf{I}_{12}
\end{array}\right) \quad \text { where } \quad \mathcal{B}:=\left(\begin{array}{cc}
0 & \mathbf{e}^{\mathrm{t}} \\
-\mathbf{e} & \mathcal{C}
\end{array}\right)
$$

$\mathbf{e}=(1, \ldots, 1)^{\mathrm{t}}, \mathcal{C}:=\left(\left(\frac{k-l}{11}\right)\right)_{1 \leq k, l \leq 11}$ and $\left(\frac{\dot{\overline{11}}}{11}\right)$ is the Legendre symbol mod 11 we obtain 


$$
\zeta\left(s ; \mathcal{L}_{24}\right)=\frac{65520}{691}(\zeta(s) \zeta(s-11)-L(s, \Delta)) .
$$

For a more general representation of Epstein zeta-functions we only sketch an approach. In particular for definitions of Eisenstein series, modular forms and cusp forms we refer to Iwaniec [37]. We first define the number

$$
r(m ; \mathcal{Q}):=\#\left\{\mathbf{x} \in \mathbb{Z}^{n}: \mathcal{Q}[\mathbf{x}]=m\right\},
$$

counting how often the associated quadratic form $\mathcal{Q}$ takes on a certain value $m$. Then we can express an Epstein zeta-function by

$$
\zeta(s ; \mathcal{Q})=\sum_{k=1}^{\infty} \frac{r(m ; \mathcal{Q})}{k^{s}} .
$$

Now it is known, see for example Fomenko [20], that the corresponding theta series

$$
\theta(z ; \mathcal{Q})=\sum_{k=0}^{\infty} r(m ; \mathcal{Q}) \cdot e^{2 \pi i k z}
$$

in this case becomes a modular form of weight $\frac{n}{2}$, which decomposes into the summation of an Eisenstein series and a cusp form. To give some more detail we have

$$
\theta(z ; \mathcal{Q})=E_{\mathcal{Q}}(z)+S_{\mathcal{Q}}(z)
$$

where $E_{\mathcal{Q}}(z):=\sum_{k=0}^{\infty} e_{\mathcal{Q}}(k) \cdot e^{2 \pi i k z}$ is an Eisenstein series and $S_{\mathcal{Q}}(z):=$ $\sum_{k=1}^{\infty} s_{\mathcal{Q}}(k) \cdot e^{2 \pi i k z}$ is a cusp form. It is also known that the coefficients $s_{\mathcal{Q}}(k)$ obey

$$
s_{\mathcal{Q}}(k) \ll\left\{\begin{array}{lll}
k^{\frac{n}{4}-\frac{1}{2}+\epsilon} & \text { if } \mathrm{n} & \text { is even } \\
k^{\frac{n}{4}-\frac{1}{4}+\epsilon} & \text { if } \mathrm{n} & \text { is odd. }
\end{array}\right.
$$

So the Epstein zeta-function can be expressed by

$$
\zeta(s ; \mathcal{Q})=\hat{E}_{\mathcal{Q}}(s)+\hat{S}_{\mathcal{Q}}(s),
$$

with $\hat{E}_{\mathcal{Q}}(s)$ and $\hat{S}_{\mathcal{Q}}(s)$ defined as

$$
\hat{E}_{\mathcal{Q}}(s):=\sum_{k=1}^{\infty} \frac{e_{\mathcal{Q}}(k)}{k^{s}}, \quad \hat{S}_{\mathcal{Q}}(s):=\sum_{k=1}^{\infty} \frac{s_{\mathcal{Q}}(k)}{k^{s}} .
$$

Therefore $\zeta(s ; \mathcal{Q})$ is decomposable into the summation of the $L$-function associated to the Eisenstein series and the $L$-function associated to the cusp form. We leave the analysis at this point, further details on the decomposition 
of the Eisenstein series occurring in (3.15) can be found in [52], [34] and [37].

To summarize, there exists an Euler product representation only for the Epstein zeta-functions associated with binary quadratic forms and class number one $(n=2)$ or the Riemann zeta-function corresponding to the trivial case $(n=1)$. For more than two variables of the associated quadratic form we get in general at most an additive structure of more well-known objects. In the last section of this chapter we take a look at the zero distribution in general, first concentrating on the real parts of the zeros.

\subsection{Zero distribution of Epstein zeta-functions}

In this section we recall results of the zero distribution of Epstein zetafunctions. Naturally we are again concerned with the nontrivial zeros $\varrho=\beta+i \gamma$. The trivial ones for any Epstein zeta-function are located at $s=-m, \quad m \in \mathbb{N}$, which can be derived by the functional equation (1.2), see Chapter 1.

\subsubsection{Zero distribution for $n \leq 2$}

We start again with the simplest case $n=1$. Here we deal essentially with the Riemann zeta-function, see (3.8). Given the Riemann hypothesis is true, we expect all nontrivial zeros of $\zeta(s)$ to have real part $1 / 2$. Therefore, with $s=\sigma+i t$ denoting the complex variable, the associated Epstein zeta-function should have all nontrivial zeros on the critical line $\sigma=1 / 4$. If additionally the analogue of the Riemann hypothesis for Dirichlet $L$-functions is true, also (3.6), i.e. the Epstein zeta-function related to the Gaussian field, will carry all nontrivial zeros on the critical line, which is since we have moved to $n=2$, the line $\sigma=1 / 2$. Unconditionally we know that a positive proportion of the nontrivial zeros of $\zeta(s ; \mathcal{Q})$ lie on these critical lines following from the same fact for the Riemann zeta-function $\zeta(s)$, see [72], and the formula for the number of zeros for Epstein zeta-functions overall (1.3).

For binary quadratic forms the functions $\zeta(s ; \mathcal{Q})$ and $\zeta\left(s ; \mathcal{Q}^{-1}\right)$ are equal up to a constant factor. Their zero-distribution was first investigated by Potter and Titchmarsh [54] who proved that infinitely many zeros lie on the critical line $\sigma=1 / 2$. Also for $n=2$ Bateman and Grosswald [4] showed that $\zeta(s ; \mathcal{Q})$ has a real zero between $1 / 2$ and 1 if

$$
k:=\frac{\sqrt{|D|}}{2 a}>7.00556
$$

with $D$ and $a$ defined as in (3.2) and (3.1). We note that this result was also announced by Chowla and Selberg [9] but they never published a proof. 
Other examples of zeros off the critical line were found by Arenstorf and Brewer [2], who studied the form (3.1) with $a=1, b=0$ and $c$ varying between 1 and 6. Deuring [13] and Stark [64] showed that all zeros of Epstein zeta-functions for $n=2$ in the rectangle $-1<\operatorname{Re} s<2,|\operatorname{Im} s| \leq 2 k$ lie on the critical line $\sigma=1 / 2$ and are simple with the exception of two real zeros between 0 and 1 , provided $k$ is sufficiently large.

If the class number is larger than one, $\zeta(s, \mathcal{Q})$ has an infinitude of zeros in the half-plane $\operatorname{Re} s>1$ as was shown by Davenport and Heilbronn [11]. Hejhal [35] and Bombieri and Hejhal [6] showed that almost all zeros of Epstein zeta-functions associated with binary quadratic forms lie on the critical line provided that the Generalized Riemann hypothesis and a widely accepted conjecture on the spacing of zeros of $L$-functions for ideal class characters is true. Recently, Rezyakova [57] showed that a positive proportion of the zeros of any $\zeta(s, \mathcal{Q})$ with a binary quadratic form $\mathcal{Q}$ independent of the class number lie on the critical line. For class number 2, Bombieri and Müller [7] obtained upper and lower bounds for the asymptotic rate of approach of zeros to the boundary of the zero-free half-plane for certain Epstein zeta-functions. For class number greater than 1 and negative discriminant, Gonek and Lee [30] derived, building on results of Lee [45] and Voronin [77], an estimate for $N\left(\sigma_{1}, \sigma_{2} ; T\right)$, the number of nontrivial zeros $\varrho=\beta+i \gamma$ with $|\gamma| \leq T$ and $\sigma_{1}<\beta<\sigma_{2}$. They showed that for any pair $\sigma_{1}, \sigma_{2}$ with $1 / 2<\sigma_{1}<\sigma_{2}<1$ the asymptotic formula

$$
N\left(\sigma_{1}, \sigma_{2} ; T\right)=c_{1} T+O\left(T \cdot e^{-c_{2} \sqrt{\log \log T}}\right)
$$

holds with a constant $c_{1}$ depending on the form $\mathcal{Q}$ and $\sigma_{1}, \sigma_{2} ; c_{2}$ being an absolute constant. Finally for class number greater than one Lee [46] gave recently an asymptotic for the number of zeros in the region $\sigma>1 / 2+\log T^{-\theta}$ for $T<\operatorname{Im} s<2 T$ with $0<\theta<1$.

\subsubsection{Zero distribution for $n>2$}

The nature of the distribution of zeros for Epstein zeta-functions changes if we consider quadratic forms for more than two variables. In general the clustering around the critical line falls apart, but we also need to note that in many cases little can be said about the distribution at all. In case of the above mentioned examples (3.11) for $n=4$ and (3.12) for $n=8$ we expect, due to the Riemann zeta-function as main ingredient, zeros on the lines $\sigma=1 / 2$ and $\sigma=3 / 2$, respectively $\sigma=1 / 2$ and $\sigma=7 / 2$. In the former example infinitely many zeros lie additionally on the line $\sigma=2$. For both examples there are no zeros on the respective critical lines $\sigma=1$ and $\sigma=2$ since all nontrivial zeros of $\zeta(s)$ are in the critical strip $0<\operatorname{Re} s<1$, see for example [72]. Looking at example (3.13) nothing definite can be said about 
the location of the zeros.

For $n=4$ Fujii [21] investigated the real zeros of Epstein zeta-functions with $\mathcal{Q}[\mathbf{x}]=x_{1}^{2}+x_{2}^{2}+d\left(x_{3}^{2}+x_{4}^{2}\right)$. Terras [71] gave examples which have real zeros off the critical line $\sigma=n / 4$ for arbitrary $n$. Furthermore Travĕnec and Samaj [73] recently derived a method to generate off-critical zeros for all sufficiently large dimensions of hypercubic Epstein zeta-functions.

Thanks to results from Steuding [65] we know that the mean value of the real parts of the nontrivial zeros is equal to the abscissa of the critical line $\sigma=n / 4$. More precisely, recalling the definition for the minimum value $\mathrm{m}(\mathcal{Q})$ of a quadratic form $(1.4)$ and with $N(\mathcal{Q})$ counting the number of $\mathbf{x}$ for which $\mathcal{Q}[\mathbf{x}]=m(\mathcal{Q})$, we have

$$
\sum_{|\gamma|<T}\left(\beta-\frac{n}{4}\right)=-\frac{T}{\pi} \Sigma(\mathcal{Q})+O(\log T)
$$

with

$$
\Sigma(\mathcal{Q}):=\log \left((\operatorname{det} \mathcal{Q})^{\frac{1}{2}} \frac{N(\mathcal{Q})}{N\left(\mathcal{Q}^{-1}\right)}\left(\frac{m\left(\mathcal{Q}^{-1}\right)}{m(\mathcal{Q})}\right)^{\frac{n}{4}}\right) .
$$

$\Sigma(\mathcal{Q})$ measures the asymmetries in the zero-distribution with respect to the critical line. For binary quadratic forms this quantity is always zero, on the other hand for instance for the matrix

$$
\mathcal{Q}=\left(\begin{array}{lll}
1 & 0 & 0 \\
0 & 2 & 0 \\
0 & 0 & 3
\end{array}\right)
$$

we find $\Sigma(\mathcal{Q})=\frac{1}{4} \log \frac{4}{3} \neq 0$. Generally, if $\Sigma(\mathcal{Q})$ is nonzero, $\zeta(s ; \mathcal{Q})$ has infinitely many zeros off the critical line $\sigma=n / 4$. Compared to the number of zeros overall, the possible asymmetries are small. We see this by combining (1.3) and (3.16) to obtain

$$
\frac{1}{N(T ; \mathcal{Q})} \sum_{|\gamma|<T} \beta=\frac{n}{4}+O\left(\frac{1}{\log T}\right) .
$$

Another result about the zero-distribution for Epstein zeta-functions is from Nakamura and Pańkowski [52]. They showed that under certain conditions for $\mathcal{Q}$, if $n \geq 4$ is even, the Epstein zeta-function has at least $C T$ zeros for some positive constant $C$ in the region $\sigma>\frac{n-1}{2}$. As a corollary they derived for the $2 k$-dimensional unit matrix $I_{2 k}$ with $k \in \mathbb{N}$ and $k \neq 1,2,4$ that $\zeta\left(s ; \mathbf{I}_{2 k}\right)$ have complex zeros in the strip $k-\frac{1}{2}<\sigma<k$, also located off the critical line. We close this section with an interesting result based on Siegel [62] and improved by Steuding [66]. It says that for certain functions related to the unit matrix in higher dimensions $\zeta(s ; \mathcal{Q})$ has only a small number of zeros around 
3 Theory of Epstein zeta-functions

the critical line $\sigma=n / 4$. In particular, almost all zeros of $\zeta\left(s ; \mathbf{I}_{n}\right)$ with $n \geq 12$ lie in the strips $0 \leq \sigma<2$ and $\frac{n}{2}-2<\sigma \leq \frac{n-1}{2} \epsilon$ for any $\epsilon>0$. 


\section{Landau's theorem}

"Wir Mathematiker sind alle ein bißchen meschugge."

Edmund Landau (1877-1938)

As indicated already in Chapter 1, Landau's theorem plays an important role in this thesis. We will first take a closer look at his original paper [43] and sketch the idea of proof. Then, in sections 4.2 and 4.3 , we will briefly recall variants and generalizations of the theorem with an emphasis on uniform versions which will become more significant in the analysis of discrepancy in Chapter 6. Finally, in section 4.4 we explore known results of zeta-functions where the theorem was used to derive uniform distribution modulo one of zero ordinates, respectively similar results from value distribution theory.

\subsection{Landau's theorem in his original paper}

Landau published his paper "Über die Nullstellen der Zetafunktion" [43] in 1912. He referred to von Mangoldts famous result, that for the nontrivial zeros $\varrho=\beta+\gamma i$ of the Riemann zeta-function the series

$$
\sum_{\varrho} \frac{x^{\varrho}}{\varrho}
$$

converges for all $x \in \mathbb{R}^{+} \backslash\{1\}$. Taking this as a starting point he introduces the three series

$$
\sum_{\varrho} \frac{x^{\varrho}}{\varrho^{\nu}}, \quad \sum_{\gamma>0} \frac{x^{\varrho}}{\varrho^{\nu}}, \quad \sum_{\gamma<0} \frac{x^{\varrho}}{\varrho^{\nu}}
$$

with a new parameter $\nu \in(0,1]$. His aim is to study their asymptotic behaviour. The sums, as usual, run in ascending order of the absolute value 
of the imaginary parts $\gamma$ of the zeros. In the first of these series he excludes the case $\nu=1$ since this is the same as (4.1). He then puts the analysis down to study the divergence of the series in the limiting case $\nu=0$. Since the zeros of the Riemann zeta-function occur in conjugate complex pairs, Landau can restrict the analysis to the series $\sum_{\gamma>0} x^{\varrho}$.

This is where the theorem arises in his paper. Equivalent to (1.6), the original formulation runs

Theorem 4.1 (Landau, 1912). For a fixed $x>1$ there is

$$
\sum_{0<\gamma \leq T} x^{\varrho}= \begin{cases}-\frac{T}{2 \pi} \log p+O(\log T) & \text { for } x \text { a prime power } p^{m}, \\ O(\log T) & \text { for } x \text { not a prime power } p^{m} .\end{cases}
$$

If $1<x_{0}<x_{1}$ and the interval $\left[x_{0}, x_{1}\right]$ is free of a prime power $p^{m}$, then for $x_{0} \leq x \leq x_{1}$

$$
\sum_{0<\gamma \leq T} x^{\varrho}=O(\log T)
$$

holds uniformly.

The proof employs Cauchy's theorem applied to

$$
\int x^{s} \frac{\zeta^{\prime}(s)}{\zeta(s)} d s
$$

The integration path is the counterclockwise oriented rectangle with edges $2+q i, 2+T i, z+T i, z+q i$, where $z<0,0<q<2$ and $T \geq 2$. Here $q$ is chosen less than the smallest ordinate $\gamma$ of $\varrho$. Assuming that the parameter $T$ avoids zeros of $\zeta(s)$, which translate to poles of the logarithmic derivative $\frac{\zeta^{\prime}(s)}{\zeta(s)}$, he obtains

$$
\int_{2+q i}^{2+T i} x^{s} \frac{\zeta^{\prime}(s)}{\zeta(s)} d s=\left\{\int_{2+q i}^{z+q i}+\int_{z+q i}^{z+T i}+\int_{z+T i}^{2+T i}\right\} x^{s} \frac{\zeta^{\prime}(s)}{\zeta(s)} d s+2 \pi i \sum_{0<\gamma<T} x^{\varrho} .
$$

Landau finds that the first and second integral on the right-hand side stay bounded for growing $T$ by taking the logarithmic derivative of the functional equation of $\zeta(s)$ and showing that

$$
\left|x^{s} \frac{\zeta^{\prime}(s)}{\zeta(s)}\right|=O\left(x^{\sigma} \log (-\sigma+t)\right)
$$

holds in the area $\sigma \leq 1, t \geq q$ of the complex plane. He further proves that

$$
\int_{z+T i}^{2+T i} x^{s} \frac{\zeta^{\prime}(s)}{\zeta(s)} d s=O(\log T)
$$


for any fixed $z<0$ and that the integral on the right-hand side delivers the asymptotic of the theorem. In particular he obtains

$$
x^{s} \frac{\zeta^{\prime}(s)}{\zeta(s)}=x^{s} \sum_{p, m} \frac{-\log p}{p^{m s}}=-\sum_{p, m}\left(\frac{x}{p^{m}}\right)^{s} \log p .
$$

Hence, by case differentiation for the parameter $x$ between prime powers and non-prime powers, the integrand becomes constant exactly when $x$ equals a prime power $p^{m}$. Therefore, he gets

$$
\int_{2+q i}^{2+T i} x^{s} \frac{\zeta^{\prime}(s)}{\zeta(s)} d s= \begin{cases}-T i \log p+O(1) & \text { for } \quad x=p^{m} \\ O(1) & \text { for } \quad x \neq p^{m}\end{cases}
$$

In principle similar techniques are often applied to prove generalizations of Landau's theorem. Some of them we will consider in the rest of this chapter and beyond, when we will turn our attention back to Epstein zeta-functions.

\subsection{Applications and generalizations}

Landau's theorem and variations of it have been used, for example by Fujii [22], in a series of papers. He evaluates discrete moments of the zeta-function or its derivative near or at its zeros, for instance $\sum_{0<\gamma<T} \zeta^{\prime}\left(\frac{1}{2}+i \gamma\right)$ with precise error terms under the assumption of the Riemann hypothesis. Fujii [23] used it also to investigate the sequence $\gamma+\gamma^{\prime}$ with both $\gamma$ and $\gamma^{\prime}$ ranging through the set of positive ordinates of zeta zeros in ascending order. Again assuming the Riemann hypothesis, he obtained the asymptotic formula

$$
\sum_{\substack{0<\gamma, \gamma^{\prime}<T \\ \gamma+\gamma^{\prime}<T}} x^{i\left(\gamma+\gamma^{\prime}\right)}=\frac{\Lambda(x)^{2}}{x} \frac{T^{2}}{8 \pi^{2}}+\frac{x^{i T}}{\log x} \frac{T(\log T)^{2}}{4 \pi^{2} i}+o\left(T^{2}(\log T)^{2}\right) .
$$

Since the number of terms $\gamma+\gamma^{\prime}<T$ is asymptotically equal to $\frac{T^{2}(\log T)^{2}}{8 \pi^{2}}$ one can divide (4.3) by the number of zeros and apply Weyl's criterion Theorem 2.2. It follows that the sequence of $\gamma+\gamma^{\prime}$ is uniformly distributed modulo one. Egami and Matsumoto [14] used this to motivate a related conjecture on distances between zero ordinates in order to show that a certain multiple zetafunction has a natural boundary. Another path of generalization was followed by Kaczorowski, Languasco and Perelli, who introduced weights in order to obtain an error term of more flexible shape, see [38].

\subsection{Uniform versions of Landau's theorem}

Since Landau's theorem lacks uniformity in $x$, the use of it is somehow limited. Therefore we are interested in a version that is uniform in both variables $x$ 
and $T$, which will for instance enable us to study discrepancy, see Chapter 6. Gonek [28], [29] obtained first results in this direction and proved that for $T>1$

$$
\begin{aligned}
\sum_{0<\gamma \leq T} x^{\varrho} & =-\frac{T}{2 \pi} \Lambda(x)+O(x \log (2 x T) \log \log 3 x) \\
& =O\left(\log x \min \left\{T ; \frac{x}{\langle x\rangle}\right\}\right)+O\left(\log (2 T) \min \left\{T ; \frac{1}{\log x}\right\}\right) .
\end{aligned}
$$

Here $\langle x\rangle$ denotes the distance between $x$ and the nearest prime power other than $x$ itself. Provided $x$ is fixed Landau's theorem 4.1 follows from (4.4) by letting $T \rightarrow \infty$. If $x=n \in \mathbb{N}$ and $T \gg n$, then the last two error terms in (4.4) are absorbed in the first one and (4.4) becomes

$$
\sum_{0<\gamma \leq T} n^{\varrho}=-\frac{T}{2 \pi} \Lambda(n)+O(n \log (2 n T) \log \log 3 n) .
$$

The last two error terms in (4.4) exhibit the contribution of the spikes of the sum of zeta-zeros when $x$ is a real number near to a prime power.

Additionally, assuming the Riemann hypothesis, Fujii [24], [25] gave a stronger version of (4.4).

\subsection{Uniform distribution modulo one for various zeta-functions}

As shown in section 2.3 uniform distribution modulo one of zero ordinates holds for the Riemann zeta-function. Due to the structure of Landau's theorem 4.1, giving an asymptotic for the sum over the exponential function $e^{\log x(\beta+i \gamma)}$, it is natural to search for similar results for further functions and derive uniform distribution from Weyl's criterion Theorem 2.2.

Already Hlawka [36] noticed that the results for the Riemann zeta-function carry over to Dirichlet $L$-functions of residue class characters. Landau himself initiated another direction of research, again starting with the analysis of $\zeta(s)$ concerning value-distribution. At the fifth International Mathematical Congress in Cambridge in 1912 he put up the question of where the roots of $\zeta(s)=a$ lie for an arbitrary complex number $a$. This of course includes the case of zeros for $a=0$. Steuding [67] showed that the ordinates of these $a$-points, denoted by $\varrho_{a}=\beta_{a}+i \gamma_{a}$, are also uniformly distributed modulo one by proving the following variant of Landau's theorem. For $x \in \mathbb{R}^{+} \backslash\{1\}$ and $T \rightarrow \infty$ we have 


$$
\sum_{0<\gamma_{a} \leq T} x^{\varrho_{a}}=\left(\alpha(x)-x \Lambda\left(\frac{1}{x}\right)\right) \frac{T}{2 \pi}+O\left(T^{\frac{1}{2}+\epsilon}\right) .
$$

Here $\alpha(x)$ equal, if $x=n$ or $x=1 / n$ for some integer $n \geq 2$, the Dirichlet series coefficients defined for $a \neq 0$ via

$$
\frac{\zeta^{\prime}(s)}{\zeta(s)-a}=\sum_{n \geq 2} \frac{\alpha(n)}{n^{s}}
$$

and zero otherwise.

Furthermore Garunkštis, Šimènas and Steuding [31] showed the equivalent result for the $a$-points of the Selberg function associated with a compact Riemann surface.

For the remainder of this chapter we will recall an important result for our purpose from the paper of Akbary and Murty [1]. They studied a broader class of Dirichlet series generalizing the Selberg class, for which they were also able to show that the zero ordinates $\gamma_{n}$ are uniformly distributed modulo one. A crucial necessary assumption for this result is a hypothesis concerning the density of the zeros on average, which we will define below.

Let $\tilde{\mathcal{S}}$ be the class of Dirichlet series

$$
F(s)=\sum_{k=1}^{\infty} \frac{a_{n}}{n^{s}}, \quad a_{1}=1
$$

which satisfy the following conditions:

- (Euler product) $F(s)$ has multiplicative coefficients and is absolutely convergent for $\operatorname{Re} s>1$, i.e.

$$
F(s)=\sum_{n=1}^{\infty} \frac{a_{n}}{n^{s}}=\prod_{p}\left(\sum_{k=0}^{\infty} \frac{a_{p^{k}}}{p^{k s}}\right)=\prod_{p} F_{p}(s), \quad \text { for } \quad \operatorname{Re} s>1 .
$$

- $F_{p}(s) \neq 0$ on $\operatorname{Re}(s)>1$, for any $p$.

- (Analytic continuation) For some integer $m \geq 0,(s-1)^{m} F(s)$ extends to an entire function of finite order.

- (Functional equation) There are numbers $Q>0, \alpha_{j}>0, r_{j} \in \mathbb{C}$ such that

$$
\Phi(s)=Q^{s} \prod_{j=1}^{d} \Gamma\left(\alpha_{j} s+r_{j}\right) F(s)
$$

satisfies the functional equation

$$
\Phi(s)=\epsilon \bar{\Phi}(1-s)
$$

where $\epsilon$ is a complex number with $|\epsilon|=1$ and $\bar{\Phi}(s)=\overline{\Phi(\bar{s})}$. 
From these conditions it follows that $\log F_{p}(s)$ has a Dirichlet series representation of the form

$$
\log F_{p}(s)=\sum_{k=1}^{\infty} \frac{b_{p^{k}}}{p^{k s}}, \quad \text { for } \quad \operatorname{Re}(s)>c_{p},
$$

where $c_{p}$ is a positive number which depends on $p$. The coefficients $b_{p^{k}}$ are given by the recursion

$$
b_{p^{k}}=a_{p^{k}}-\frac{1}{k} \sum_{j=1}^{k-1} j \cdot b_{p^{j}} \cdot a_{p^{k-j}},
$$

where $b_{p}=a_{p}$, see Lemma 1 in [1].

Then there exists the following generalization of Landau's theorem to $F \in \tilde{\mathcal{S}}$, see [50]: For $x>1$ and $T \rightarrow \infty$

$$
\sum_{0<\gamma \leq T} x^{\varrho}=-\frac{T}{2 \pi} \Lambda_{F}(x)+O_{F, x}(\log T)
$$

where

$$
\Lambda_{F}(x)= \begin{cases}b_{x} \log x & \text { if } x=p^{k} \\ 0 & \text { otherwise }\end{cases}
$$

Now $F \in \tilde{\mathcal{S}}$ satisfies the Average Density Hypothesis if for $T \rightarrow \infty$

$$
\sum_{\substack{0 \leq \gamma \leq T \\ \beta>\frac{1}{2}}}\left(\beta-\frac{1}{2}\right)=o\left(N_{F}(T)\right)
$$

where $N_{F}(T)$ denotes the number of zeros of $F$ with $0 \leq \beta \leq 1$ and $0 \leq \gamma \leq T$.

Building on these definitions Akbary and Murty arrive at their main result, Theorem 6:

Theorem 4.2. Let $F \in \tilde{\mathcal{S}}$. Suppose that $F$ satisfies the Average Density Hypothesis, then for $\alpha \neq 0,\left(\alpha \gamma_{n}\right)_{n \in \mathbb{N}}$ is uniformly distributed modulo one.

The Average Density Hypothesis ensures that most of the zeros lie on the critical line. This property is of course valid for the Riemann zeta-function, see section 2.3 and the proof for uniform distribution modulo one runs analogously to what we recalled there. Additionally Akbary and Murty introduced the socalled Moment Hypothesis which is satisfied if there exists a real $k>0$ such that

$$
M_{F}(k, T)=\frac{1}{T} \int_{0}^{T}|F(1 / 2+i t)|^{2 k} d t=O_{k, F}\left(e^{\psi(T)}\right)
$$


for some $\psi(T)$, where $\psi(T)$ is a positive real function such that $\psi(T)=o(\log T)$. The Average Density Hypothesis and therefore Theorem 4.2 follows from the Moment Hypothesis. As examples of functions in $\tilde{\mathcal{S}}$ for which the Moment Hypothesis holds for $k=1$ we recall the remarks from section 5 of Akbary and Murty [1], the exact definitions can be found in the mentioned references: For a primitive Dirchlet character $\bmod q$ its associated Dirichlet $L$-series $L(s, \chi)$, the $L$-series $L(s, f)$ associated to a holomorphic cusp newform of weight $k$ and level $N$ with nebentypus $\phi$, and the $L$-series $L(s, g)$ associated to an even Maas cusp newform of weight zero and level $N$ with nebentypus $\phi$. The sufficient asymptotics are stated for $L(s, \chi)$ in Motohashi [51], for $L(s, f)$ in Zhang [81] and for $L(s, g)$ in Zhang [82].

In the next chapter we will use the concept of a density assumption plus a variant of Landau's theorem to derive similar results for the general Epstein zeta-function. 



\title{
Uniform distribution of imaginary parts of zeros of Epstein zeta-functions
}

\author{
"Die Mehrheit bringt der Mathematik Gefühle entgegen, wie sie nach \\ Aristoteles durch die Tragödie geweckt werden sollen, nämlich Mitleid \\ und Furcht. Mitleid mit denen, die sich mit der Mathematik plagen \\ müssen, und Furcht: daß man selber mal in diese gefährliche Lage \\ geraten könne."
}

Paul Epstein (1883-1966)

Chapter 5 contains the main results of this thesis. First we introduce supporting quantities and properties about Epstein zeta-functions. In Lemma 5.1 we gain an explicit representation of the logarithmic derivative of the modified Epstein zeta-function $Z(s ; \mathcal{Q})$. We continue to prove the central result Theorem 5.3, the analogue of Landau's theorem. Incorporating the results of Chapter 2 we prove Corollaries 5.4 and 5.5 in order to show uniform distribution modulo one of the zero ordinates under the according density assumptions. We further apply these results to binary quadratic forms, Corollary 5.6, and expose examples where the necessary conditions hold for quadratic forms in more than two variables. We derive a recursive representation of the logarithmic derivative of $Z(s ; \mathcal{Q})$ and close the chapter by discussing shortly open questions. Except Corollaries 5.5 and 5.6 these results can be found in [59].

\subsection{Preliminaries}

In order to facilitate the analysis we first define the function

$$
Z(s ; \mathcal{Q}):=\frac{\mathrm{m}(\mathcal{Q})^{s}}{\mathrm{~N}(\mathcal{Q})} \zeta(s ; \mathcal{Q})
$$

where $\mathrm{N}(\mathcal{Q})$ again counts the number of $\mathbf{x}$ for which $\mathcal{Q}[\mathbf{x}]=\mathrm{m}(\mathcal{Q})$, the minimal non-zero value of the quadratic form, see definitions in (1.4) and section 3.5.2. 
It is important that $Z(s ; \mathcal{Q})$ carries the same zeros as $\zeta(s ; \mathcal{Q})$, i.e. $Z(s ; \mathcal{Q})=0$ is equivalent to $\zeta(s ; \mathcal{Q})=0$. In view of the functional equation $(1.2)$,

$$
Z(s ; \mathcal{Q})=\frac{\mathrm{m}(\mathcal{Q})^{s}}{\mathrm{~N}(\mathcal{Q})} \cdot(\operatorname{det} \mathcal{Q})^{-\frac{1}{2}} \cdot \pi^{2 s-\frac{n}{2}} \cdot \frac{\Gamma\left(\frac{n}{2}-s\right)}{\Gamma(s)} \cdot \frac{\mathrm{N}\left(\mathcal{Q}^{-1}\right)}{\mathrm{m}\left(\mathcal{Q}^{-1}\right)^{\frac{n}{2}-s}} \cdot Z\left(\frac{n}{2}-s ; \mathcal{Q}^{-1}\right)
$$

We also need to take a look at the Dirichlet series expansions of $Z(s ; \mathcal{Q})$ and also at $Z^{\prime}(s ; \mathcal{Q})$, as we will have to deal with the logarithmic derivative of $Z(s ; \mathcal{Q})$. Recalling definition $(3.14)$

$$
r(m ; \mathcal{Q}):=\#\left\{\mathbf{x} \in \mathbb{Z}^{n}: \mathcal{Q}[\mathbf{x}]=m\right\}
$$

and by defining

$$
a(n):=\frac{r(n, \mathcal{Q})}{\mathrm{N}(\mathcal{Q})}
$$

we observe that

$$
Z(s ; \mathcal{Q})=\frac{\mathrm{m}(\mathcal{Q})^{s}}{\mathrm{~N}(\mathcal{Q})} \zeta(s ; \mathcal{Q})=1+\sum_{n>\mathrm{m}(\mathcal{Q})} a(n)\left(\frac{\mathrm{m}(\mathcal{Q})}{n}\right)^{s}
$$

and

$$
Z^{\prime}(s ; \mathcal{Q})=\sum_{n>\mathrm{m}(\mathcal{Q})} a(n) \log \left(\frac{\mathrm{m}(\mathcal{Q})}{n}\right)\left(\frac{\mathrm{m}(\mathcal{Q})}{n}\right)^{s} .
$$

Before we analyse the logarithmic derivative further we establish a zero-free region of $Z(s ; \mathcal{Q})$. Following section 2 of Steuding [65], we denote by $\mathrm{m}_{2}(\mathcal{Q})$ the second minimum of $\mathcal{Q}$, i.e. the least value $>\mathrm{m}(\mathcal{Q})$ of $\mathcal{Q}[\mathbf{y}]$ for $\mathbf{y} \in \mathbb{Z}^{n}$. Setting

$$
\lambda:=\frac{\mathrm{m}_{2}(\mathcal{Q})}{\mathrm{m}(\mathcal{Q})}
$$

for $\sigma>\frac{n}{2}$, we then have, as $\sigma \rightarrow \infty$,

$$
Z(s ; \mathcal{Q})=1+c \lambda^{-s}+o\left(\lambda^{-\sigma}\right),
$$

where $c$ is a positive constant depending only on $\mathcal{Q}$. Therefore there exists a zero-free half plane to the right. The same argument yields a zero-free region for $Z\left(s ; \mathcal{Q}^{-1}\right)$, so from the functional equation (5.2) we deduce the existence of a positive constant $B_{1}$ such that neither $Z(s ; \mathcal{Q})$ nor $Z\left(s ; \mathcal{Q}^{-1}\right)$ have nontrivial zeros outside the strip $-B_{1}<\sigma<B_{1}$. It also follows from (5.5) that there exists a constant $B_{2}$ such that $Z(\sigma+i t ; \mathcal{Q})>\frac{1}{2}$ for $\sigma>B_{2}$. Additionally, if we consider the supremum and the infimum of the real parts $\beta_{i}$ of the nontrivial zeros, we certainly find a constant $B_{3}$ where both inequalities $1 \leq\left|B_{3}-\sup \beta_{i}\right|$ and $1 \leq\left|B_{3}-\inf \beta_{i}\right|$ hold. By defining $B_{3}:=\max \left\{\left|\sup \beta_{i}\right|,\left|\inf \beta_{i}\right|\right\}+1$ we ensure that these inequalities hold also for all constants larger than $B_{3}$. The 
properties of the constants $B_{i}$ are prerequisites for the proof of our main result.

The following construction is essentially due to Landau [44]. According to Garunkštis, Steuding and Šimenas [31] we consider the unbounded sequence

$$
1<\frac{\mathrm{m}(\mathcal{Q})+1}{\mathrm{~m}(\mathcal{Q})}<\frac{\mathrm{m}(\mathcal{Q})+2}{\mathrm{~m}(\mathcal{Q})}<\ldots
$$

of real numbers belonging to the general Dirichlet series $Z(s ; \mathcal{Q})$, see $(5.3)$. We can define $S$ as the set of all possible products of elements of this sequence and obtain an ordered sequence of all different numbers of $S$ as

$$
l_{n}(\mathcal{Q})=\prod_{\nu=1}^{h} \frac{\mathrm{m}(\mathcal{Q})+k_{\nu}}{\mathrm{m}(\mathcal{Q})}
$$

with $h \geq 0, k_{\nu} \in \mathbb{N}$.

Therefore Lemma 8 of [31] applied to the two general Dirichlet series $Z(s ; \mathcal{Q})$ and $Z^{\prime}(s ; \mathcal{Q})$ ensures that the quotient $\frac{Z^{\prime}(s ; \mathcal{Q})}{Z(s ; \mathcal{Q})}$ is again a general Dirichlet series which converges absolutely in a right half-plane and carries the numbers $l_{n}(\mathcal{Q})$, see (5.7), as its defining unbounded sequence. In particular we can prove the following Lemma giving an explicit representation.

Lemma 5.1. For $Z(s ; \mathcal{Q})$ and $Z^{\prime}(s ; \mathcal{Q})$ defined as above the quotient $\frac{Z^{\prime}(s ; \mathcal{Q})}{Z(s ; \mathcal{Q})}$ is again a general Dirichlet series and there exists a constant $B_{4}$ such that $\frac{Z^{\prime}(s ; \mathcal{Q})}{Z(s ; \mathcal{Q})}$ converges absolutely for $\sigma>B_{4}$. We have

$$
\frac{Z^{\prime}(s ; \mathcal{Q})}{Z(s ; \mathcal{Q})}=\sum_{n=1}^{\infty} \frac{b_{n}(\mathcal{Q})}{l_{n}(\mathcal{Q})^{s}}
$$

where the $l_{n}(\mathcal{Q})$ 's are defined in (5.7) with $l_{n}(\mathcal{Q}) \rightarrow \infty$ for $n \rightarrow \infty$. Moreover, the coefficients $b_{n}(\mathcal{Q})$ are given explicitly as

$$
\begin{aligned}
b_{n}(\mathcal{Q})= & \sum_{\text {distinct } k_{i}} a\left(\mathrm{~m}(\mathcal{Q})+k_{i}\right) \log \left(\frac{\mathrm{m}(\mathcal{Q})}{\mathrm{m}(\mathcal{Q})+k_{i}}\right) \times \\
& \times \sum_{\substack{k_{1}, \ldots, k_{h} \in \mathbb{N} \\
g_{1}+\ldots+g_{r}=h}}(-1)^{h-1}\left(\begin{array}{c}
h-1 \\
g_{1}, \cdots, g_{j-1}, g_{j}-1, g_{j+1}, \cdots g_{r}
\end{array}\right) \times \\
& \times a\left(\mathrm{~m}(\mathcal{Q})+k_{1}\right) \cdot \ldots \cdot a\left(\mathrm{~m}(\mathcal{Q})+k_{i-1}\right) \cdot a\left(\mathrm{~m}(\mathcal{Q})+k_{i+1}\right) \\
& \cdot \ldots \cdot a\left(\mathrm{~m}(\mathcal{Q})+k_{h}\right) .
\end{aligned}
$$

Here $g_{1}, \ldots, g_{r}$ are the multiplicities of the distinct values of the $k_{1}, \ldots, k_{h}$, hence $r \leq h$ and $g_{1}+\ldots+g_{r}=h, g_{j}$ is the multiplicity of $k_{i}$. 
Proof: Since $Z(s ; \mathcal{Q})$ starts with a constant term 1 , there exists an inverse of the Dirichlet series

$$
\frac{1}{Z(s ; \mathcal{Q})}=\frac{\mathrm{N}(\mathcal{Q})}{\mathrm{m}(\mathcal{Q})^{s} \zeta(s ; \mathcal{Q})}
$$

and we can apply the method of Landau [44] provided that Re $s$ is sufficiently large. More precisely, the inverse of $Z(s ; \mathcal{Q})$, i.e.

$$
\frac{1}{Z(s ; \mathcal{Q})}=\sum_{n=1}^{\infty} \frac{c_{n}(\mathcal{Q})}{l_{n}(\mathcal{Q})^{s}}
$$

is a general Dirichlet series with the $l_{n}(\mathcal{Q})$ of the form (5.7) and some $c_{n}(\mathcal{Q})$. This follows immediately from the first consideration in the Appendix of Landau [44] where in our case we set $m=\mathrm{m}(\mathcal{Q}), f(s)=\frac{\zeta(s ; \mathcal{Q})}{\mathrm{N}(\mathcal{Q})}=$ $\sum_{n=1}^{\infty} \frac{a(n)}{n^{s}}$ and $a(\mathrm{~m}(\mathcal{Q}))=1$, the first non-zero coefficient. Hence, the product $Z^{\prime}(s ; \mathcal{Q})^{n^{s}} \times \frac{1}{Z(s ; \mathcal{Q})}$ of two general Dirichlet series is again a Dirichlet series of the form

$$
\frac{Z^{\prime}(s ; \mathcal{Q})}{Z(s ; \mathcal{Q})}=\sum_{n=1}^{\infty} \frac{b_{n}(\mathcal{Q})}{l_{n}(\mathcal{Q})^{s}}
$$

Taking into account the uniqueness theorem for Dirichlet series, there exists a zero-free half-plane. Accordingly, we may find a positive number $B_{4}$ such that the abscissa of absolute convergence of the Dirichlet series above is less than or equal to $B_{4}$. Explicitly we obtain

$$
\begin{aligned}
\frac{1}{Z(s ; \mathcal{Q})}= & \sum_{h=0}^{\infty}(-1)^{h}\left(\sum_{n>\mathrm{m}(\mathcal{Q})}^{\infty} a(n)\left(\frac{\mathrm{m}(\mathcal{Q})}{n}\right)^{s}\right)^{h} \\
= & \sum_{h=0}^{\infty}(-1)^{h} \sum_{\substack{k_{1}, \ldots, k_{h} \in \mathbb{N} \\
g_{1}+\ldots+g_{r}=h}}\left(\begin{array}{c}
h \\
g_{1} \ldots g_{r}
\end{array}\right) \times \\
& \times a\left(\mathrm{~m}(\mathcal{Q})+k_{1}\right) \cdot \ldots \cdot a\left(\mathrm{~m}(\mathcal{Q})+k_{h}\right) \times \\
& \times\left(\frac{\mathrm{m}(\mathcal{Q})^{h}}{\left(\mathrm{~m}(\mathcal{Q})+k_{1}\right) \cdot \ldots \cdot\left(\mathrm{m}(\mathcal{Q})+k_{h}\right)}\right)^{s}
\end{aligned}
$$

with the tuple $g_{1}, \ldots, g_{r} \in \mathbb{N}$ running through all partitions $P(h)$ of $h$. Hence,

$$
\begin{aligned}
\frac{Z^{\prime}(s ; \mathcal{Q})}{Z(s ; \mathcal{Q})}= & \sum_{n>\mathrm{m}(\mathcal{Q})} a(n) \log \left(\frac{\mathrm{m}(\mathcal{Q})}{n}\right)\left(\frac{\mathrm{m}(\mathcal{Q})}{n}\right)^{s} \times \\
& \times \sum_{h=0}^{\infty}(-1)^{h} \sum_{\substack{k_{1}, \ldots, k_{h} \in \mathbb{N} \\
g_{1}+\ldots+g_{r}=h}}\left(\begin{array}{c}
h \\
g_{1} \ldots g_{r}
\end{array}\right) \times \\
& \times a\left(\mathrm{~m}(\mathcal{Q})+k_{1}\right) \cdot \ldots \cdot a\left(\mathrm{~m}(\mathcal{Q})+k_{h}\right) \times \\
& \times\left(\frac{\mathrm{m}(\mathcal{Q})^{h}}{\left(\mathrm{~m}(\mathcal{Q})+k_{1}\right) \cdot \ldots \cdot\left(\mathrm{m}(\mathcal{Q})+k_{h}\right)}\right)^{s} .
\end{aligned}
$$


Now for a given $l_{n}(\mathcal{Q})$ we see that the coefficient $b_{n}(\mathcal{Q})$ takes the form as stated above. This holds since in (5.9), which shows the factors of the product $Z^{\prime}(s ; \mathcal{Q}) \cdot \frac{1}{Z(s ; \mathcal{Q})}$ separately, for a distinct $k_{i}$ always the coefficient of one summand of $Z^{\prime}(s ; \mathcal{Q})$ and the coefficient of

$$
\left(\frac{\mathrm{m}(\mathcal{Q})^{h-1}}{\left(\mathrm{~m}(\mathcal{Q})+k_{1}\right) \cdot \ldots \cdot\left(\mathrm{m}(\mathcal{Q})+k_{i-1}\right)\left(\mathrm{m}(\mathcal{Q})+k_{i+1}\right) \cdot \ldots \cdot\left(\mathrm{m}(\mathcal{Q})+k_{h}\right)}\right)^{s}
$$

contribute to $b_{n}(\mathcal{Q})$. From this we obtain the representation of the Lemma concluding the proof.

Since the Dirichlet series representing the logarithmic derivative of $Z(s ; \mathcal{Q})$ converges we are now able to choose the constant

$$
B=\max \left(B_{1}, B_{2}, B_{3}, B_{4}\right)
$$

carrying all properties needed for the proof of our main result. We also recall Lemma 5 from [31] which will be very useful.

Lemma 5.2. If $f(s)$ is analytic and $f\left(s_{0}\right) \neq 0$ with

$$
\left|\frac{f(s)}{f\left(s_{0}\right)}\right|<e^{M}
$$

in $\left\{s:\left|s-s_{0}\right| \leq r\right\}$ with $M>1$, then

$$
\left|\frac{f(s)}{f\left(s_{0}\right)}-\sum_{\varrho} \frac{1}{s-\varrho}\right|<C \frac{M}{r}
$$

for $\left|s-s_{0}\right| \leq \frac{r}{4}$, where $C$ is some constant and $\varrho$ runs through the zeros of $f(s)$ such that $\left|\varrho-s_{0}\right| \leq \frac{r}{2}$.

Finally in this section we recall a well-known result from paragraph 3 of Steuding [66] which gives information about the growth of the Epstein zeta-function. Following from the functional equation (1.2) it is based on an application of the Phragmén-Lindelöf principle : As $|t| \rightarrow \infty$, we have

$$
\zeta(s ; \mathcal{Q}) \ll|t|^{\max \left\{\frac{n}{2}-\sigma, 0\right\}+\epsilon} .
$$

\subsection{Landau's theorem for Epstein zeta-functions}

We are now in a position to give the analogue of Landau's theorem for Epstein zeta-functions. 
Theorem 5.3. Let $x \in \mathbb{R}^{+} \backslash\{1\}$. As $T \rightarrow \infty$,

$$
\sum_{|\gamma|<T} x^{\varrho}=\frac{T}{\pi}\left(\delta_{l_{k}(\mathcal{Q}), x} \cdot b_{k}(\mathcal{Q})-x^{\frac{n}{2}} \delta_{l_{k}\left(\mathcal{Q}^{-1}\right), \frac{1}{x}} \cdot b_{k}\left(\mathcal{Q}^{-1}\right)\right)+O(\log T)
$$

for all Epstein zeta-functions where $l_{k}(\mathcal{Q})$ and $b_{k}(\mathcal{Q})$ are explicitly defined in Lemma 5.1.

Here $\delta_{a, b}$ is the Kronecker symbol, equal to 1 if $a=b$ and zero otherwise. Before we turn to the proof we shall deduce from Theorem 5.3 the following two corollaries.

\subsection{Corollaries}

The first one requires a density hypothesis similar to (4.5). With the critical line at $n / 4+i t \in \mathbb{C}, t \in \mathbb{R}$ for an arbitrary Epstein zeta-function $\zeta(s ; \mathcal{Q})$ associated to a quadratic form $\mathcal{Q}$, we obtain

Corollary 5.4. If $\sum_{|\gamma|<T}\left|\beta-\frac{n}{4}\right|=o(N(T ; \mathcal{Q}))$, then the ordinates of nontrivial zeros of the Epstein zeta-function $\zeta(s ; \mathcal{Q})$ are uniformly distributed modulo one.

The proof will be provided below in section 5.4.2, it follows very closely the reasoning recalled for the Riemann zeta-function in section 2.3.

We can say a little bit more. In the next corollary we want to leverage the results of Chapter 2, in particular Lemma 2.4, which allows two different weights to appear in the limit conditions (2.8).

Corollary 5.5. Let $\left(\beta_{k}+i \gamma_{k}\right)_{k \in \mathbb{N}}$ be the sequence of nontrivial zeros of the Epstein zeta-function $\zeta(s ; \mathcal{Q})$ ordered in ascending absolute values of the imaginary parts. Let $A=\left\{a_{1}, a_{2}, \ldots\right\}$ and $B=\left\{b_{1}, b_{2}, \ldots\right\}$ be disjoint subsets of $\mathbb{N}$ with $A \cup B=\mathbb{N}$. If $\left(\beta_{k}+i \gamma_{k}\right)_{k \in \mathbb{N}}$ can be separated into two subsequences $\left(\beta_{a_{k}}+i \gamma_{a_{k}}\right)_{k \in \mathbb{N}},\left(\beta_{b_{k}}+i \gamma_{b_{k}}\right)_{k \in \mathbb{N}}$ and there exists two real numbers $\beta_{\min }, \beta_{\max }$ such that

$$
\sum_{\left|\gamma_{a_{k}}\right|<T}\left|\beta_{a_{k}}-\beta_{\min }\right|=o(N(T ; \mathcal{Q}))
$$

and

$$
\sum_{\left|\gamma_{b_{k}}\right|<T}\left|\beta_{b_{k}}-\beta_{\max }\right|=o(N(T ; \mathcal{Q})),
$$

then the ordinates of the nontrivial zeros are uniformly distributed modulo one.

This enables us to have basically two possible clustering lines of zeros where a density assumption can be applied. The proof for Corollary 5.5 is provided in 5.4.3. Examples of applications for some specific Epstein zeta-functions follow in section 5.5.2. 


\subsection{Proof of the main results}

\subsubsection{Proof of Theorem 5.3}

We aim to prove the theorem by using an appropriate contour integral and residue calculus. By choosing $b>B$ and in view of the functional equation (1.2) we ensure that all nontrivial zeros of $\zeta(s ; \mathcal{Q})$ lie inside $\operatorname{Re} s>\frac{n}{2}-b$. We further note that the logarithmic derivative of $\zeta(s ; \mathcal{Q})$ and therefore also of $Z(s ; \mathcal{Q})$ has simple poles at each zero with residue equal to the order. Therefore

$$
\sum_{|\gamma|<T} x^{\varrho}=\frac{1}{2 \pi i} \int_{\partial \mathcal{R}} x^{s} \frac{Z^{\prime}(s ; \mathcal{Q})}{Z(s ; \mathcal{Q})} d s+O(1)
$$

where the error term arises from the pole at $s=\frac{n}{2}$. Here the integration path is the counterclockwise oriented closed rectangle $\partial \mathcal{R}$ with points $B-i T$, $B+i T, \frac{n}{2}-b+i T$ and $\frac{n}{2}-b-i T$. We have

$$
\begin{aligned}
\int_{\partial \mathcal{R}} x \frac{Z^{\prime}(s ; \mathcal{Q})}{Z(s ; \mathcal{Q})} d s= & \left\{\int_{B-i T}^{B+i T}+\int_{B+i T}^{\frac{n}{2}-b+i T}+\right. \\
& \left.+\int_{\frac{n}{2}-b+i T}^{\frac{n}{2}-b-i T}+\int_{\frac{n}{2}-b-i T}^{B-i T}\right\} x^{s} \frac{Z^{\prime}(s ; \mathcal{Q})}{Z(s ; \mathcal{Q})} d s \\
= & : \sum_{j=1}^{4} I_{j}
\end{aligned}
$$

say. The idea is now to estimate all integral paths piecewise and show that each integral is $O(T) . I_{1}$ we evaluate directly in the area of absolute convergence, for $I_{3}$ we achieve the same by using the functional equation (1.2). $I_{2}$ and $I_{4}$ can be estimated by means of techniques from [31], essentially by using Lemma 5.2.

We begin with

$$
I_{1}=\int_{B-i T}^{B+i T} x^{s} \frac{Z^{\prime}(s ; \mathcal{Q})}{Z(s ; \mathcal{Q})} d s .
$$

Since summation and integration can be interchanged in the area of absolute convergence, we deduce from Lemma 5.1 that

$$
\begin{aligned}
I_{1} & =i \int_{-T}^{+T} x^{B+i t} \frac{Z^{\prime}(B+i t ; \mathcal{Q})}{Z(B+i t ; \mathcal{Q})} d t \\
& =i \int_{-T}^{+T} x^{B+i t} \sum_{n=1}^{\infty} \frac{b_{n}(\mathcal{Q})}{l_{n}(\mathcal{Q})^{(B+i t)}} d t \\
& =i \sum_{n=1}^{\infty} b_{n}(\mathcal{Q})\left(\frac{x}{l_{n}(\mathcal{Q})}\right)^{B} \int_{-T}^{+T}\left(\frac{x}{l_{n}(\mathcal{Q})}\right)^{i t} d t
\end{aligned}
$$


The integral $\int_{-T}^{+T}\left(\frac{x}{l_{n}(\mathcal{Q})}\right)^{i t} d t$ is unbounded, if and only if $\frac{x}{l_{n}(\mathcal{Q})}=1$, that is, for $x=l_{n}(\mathcal{Q})$; otherwise it is bounded and in this case we have

$$
\int_{-T}^{+T}\left(\frac{x}{l_{n}(\mathcal{Q})}\right)^{i t} d t=O(1)
$$

Putting all this together we get

$$
I_{1}=2 i \cdot \delta_{l_{k}(\mathcal{Q}), x} \cdot b_{k}(\mathcal{Q}) \cdot T+O(1)
$$

with the $b_{k}(\mathcal{Q})$ defined in Lemma 5.1.

Now we consider

$$
I_{2}=\int_{\frac{n}{2}-b+i T}^{B+i T} x^{s} \frac{Z^{\prime}(s ; \mathcal{Q})}{Z(s ; \mathcal{Q})} d s .
$$

In order to estimate this integral, we first apply Lemma 5.2. By setting the parameters

$$
f(s)=Z(s ; \mathcal{Q}), \quad s_{0}=B+i T, \quad M=C \cdot \log T, \quad r=4\left(B-\left(\frac{n}{2}-b\right)\right)
$$

with an appropriate constant $C$ we are able to meet the conditions of the Lemma since $B$ is large enough to ensure that $s_{0}=B+i T$ is from the zero-free right half plane essentially due to (5.5). Also (5.11) ensures that $\left|\frac{f(s)}{f\left(s_{0}\right)}\right|<e^{M}$ is fulfilled. We note that the constant $M$ may depend on $n$ and $\mathcal{Q}$. Hence we get

$$
\frac{Z^{\prime}(s ; \mathcal{Q})}{Z(s ; \mathcal{Q})}=\sum_{\left|\varrho_{0}-s_{0}\right| \leq \frac{r}{2}} \frac{1}{s-\varrho_{0}}+O(\log T)
$$

for $\left|s-s_{0}\right| \leq \frac{r}{4}=\left(B-\left(\frac{n}{2}-b\right)\right)$. With $\varrho_{0}=\beta_{0}+i \gamma_{0}$ it follows that

$$
\begin{aligned}
I_{2}= & \int_{\frac{n}{2}-b+i T}^{B+i T} x^{s} \frac{Z^{\prime}(s ; \mathcal{Q})}{Z(s ; \mathcal{Q})} d s \ll x^{B} \int_{\frac{n}{2}-b}^{B}\left|\frac{Z^{\prime}(\sigma+i T ; \mathcal{Q})}{Z(\sigma+i T ; \mathcal{Q})}\right| d \sigma \\
\leq & x^{B} \int_{\frac{n}{2}-b}^{B} \sum_{\left|\varrho_{0}-s_{0}\right| \leq \frac{r}{2}}\left|\frac{1}{\sigma+i T-\varrho_{0}}\right| d \sigma+O(\log T) \\
= & x^{B} \sum_{\left|\varrho_{0}-s_{0}\right| \leq \frac{r}{2}} \int_{\frac{n}{2}-b}^{B} \frac{1}{\sqrt{\left(\sigma-\beta_{0}\right)^{2}+\left(T-\gamma_{0}\right)^{2}}} d \sigma+O(\log T) \\
= & x^{B} \sum_{\left|\varrho_{0}-s_{0}\right| \leq \frac{r}{2}}\left(\log \left(B-\beta_{0}+\sqrt{\left(T-\gamma_{0}\right)^{2}+\left(B-\beta_{0}\right)^{2}}\right)\right. \\
& \left.-\log \left(\frac{n}{2}-b-\beta_{0}+\sqrt{\left(T-\gamma_{0}\right)^{2}+\left(\frac{n}{2}-b-\beta_{0}\right)^{2}}\right)\right)+O(\log T) .
\end{aligned}
$$


Now we observe that the number of zeros with $|\varrho-(B+i T)| \leq \frac{r}{2}$ is bounded by $\tilde{C} \log T$ for another constant $\tilde{C}>0$. This is true since the number of zeros for $\zeta(s ; \mathcal{Q})$ and accordingly $Z(s ; \mathcal{Q})$ is given by $(1.3)$, hence we get

$$
\begin{aligned}
N\left(T+\frac{r}{2} ; \mathcal{Q}\right)- & N\left(T-\frac{r}{2} ; \mathcal{Q}\right) \\
= & \frac{2\left(T+\frac{r}{2}\right)}{\pi} \log \frac{\left(T+\frac{r}{2}\right)}{\pi e \sqrt{\mathrm{m}(\mathcal{Q}) \mathrm{m}\left(\mathcal{Q}^{-1}\right)}} \\
& -\frac{2\left(T-\frac{r}{2}\right)}{\pi} \log \frac{\left(T-\frac{r}{2}\right)}{\pi e \sqrt{\mathrm{m}(\mathcal{Q}) \mathrm{m}\left(\mathcal{Q}^{-1}\right)}} \\
= & \frac{2 T}{\pi}\left(\log \frac{\left(T+\frac{r}{2}\right)}{\pi e \sqrt{\mathrm{m}(\mathcal{Q}) \mathrm{m}\left(\mathcal{Q}^{-1}\right)}}-\log \frac{\left(T-\frac{r}{2}\right)}{\pi e \sqrt{\mathrm{m}(\mathcal{Q}) \mathrm{m}\left(\mathcal{Q}^{-1}\right)}}\right) \\
& +\underbrace{\frac{r}{\pi} \log \frac{\left(T+\frac{r}{2}\right)}{\pi e \sqrt{\mathrm{m}(\mathcal{Q}) \mathrm{m}\left(\mathcal{Q}^{-1}\right)}}+\frac{r}{\pi} \log \frac{\left(T-\frac{r}{2}\right)}{\pi e \sqrt{\mathrm{m}(\mathcal{Q}) \mathrm{m}\left(\mathcal{Q}^{-1}\right)}}}_{O(\log T)} \\
= & \frac{2 T}{\pi}\left(\log \frac{\left(T+\frac{r}{2}\right)}{\left(T-\frac{r}{2}\right)}\right)+O(\log T)=O(\log T) .
\end{aligned}
$$

Furthermore all summands are bounded by a constant $C$ as

$$
\begin{aligned}
& \log \left(B-\beta_{0}+\sqrt{\left(T-\gamma_{0}\right)^{2}+\left(B-\beta_{0}\right)^{2}}\right) \\
& \quad-\log \left(\frac{n}{2}-b-\beta_{0}+\sqrt{\left(T-\gamma_{0}\right)^{2}+\left(\frac{n}{2}-b-\beta_{0}\right)^{2}}\right) \\
& =\log \frac{\left(B-\beta_{0}+\sqrt{\left(T-\gamma_{0}\right)^{2}+\left(B-\beta_{0}\right)^{2}}\right)}{\left(\frac{n}{2}-b-\beta_{0}+\sqrt{\left(T-\gamma_{0}\right)^{2}+\left(\frac{n}{2}-b-\beta_{0}\right)^{2}}\right)} .
\end{aligned}
$$

Since we can choose $b, B_{3}$ such that for $B>B_{3}$ we have $1 \leq\left|B-\sup \beta_{0}\right|$ and $1 \leq\left|B-\inf \beta_{0}\right|$, we also find constants $c_{1}, c_{2}$ with $c_{1}\left|\frac{n}{2}-b-\beta_{0}\right| \leq\left|B-\beta_{0}\right| \leq$ $c_{2}\left|\frac{n}{2}-b-\beta_{0}\right|$ and hence $c_{1} \leq \frac{\left|B-\beta_{0}\right|}{\left|\frac{n}{2}-b-\beta_{0}\right|} \leq c_{2}$. Therefore there exists a constant $C$ such that

$$
\log \frac{\left(B-\beta_{0}+\sqrt{\left(T-\gamma_{0}\right)^{2}+\left(B-\beta_{0}\right)^{2}}\right)}{\left(\frac{n}{2}-b-\beta_{0}+\sqrt{\left(T-\gamma_{0}\right)^{2}+\left(\frac{n}{2}-b-\beta_{0}\right)^{2}}\right)} \leq C .
$$

With this, we have shown

$$
I_{2}=\int_{\frac{n}{2}-b+i T}^{B+i T} x^{s} \frac{Z^{\prime}(s ; \mathcal{Q})}{Z(s ; \mathcal{Q})} d s=O(\log T) .
$$

Thus we can turn to

$$
I_{3}=\int_{\frac{n}{2}-b-i T}^{\frac{n}{2}-b+i T} x^{s} \frac{Z^{\prime}(s ; \mathcal{Q})}{Z(s ; \mathcal{Q})} d s
$$


To get the logarithmic derivative of $Z(s ; \mathcal{Q})$, we first take logarithms on both sides of (5.2), that is,

$$
\begin{aligned}
\log Z(s ; \mathcal{Q})= & s \log \mathrm{m}(\mathcal{Q})-\log \mathrm{N}(\mathcal{Q})+\log (\operatorname{det} \mathcal{Q})^{-\frac{1}{2}}+\left(2 s-\frac{n}{2}\right) \log \pi \\
& +\log \Gamma\left(\frac{n}{2}-s\right)-\log \Gamma(s)+\log \mathrm{N}\left(\mathcal{Q}^{-1}\right) \\
& -\left(\frac{n}{2}-s\right) \log \mathrm{m}\left(\mathcal{Q}^{-1}\right)+\log Z\left(\frac{n}{2}-s ; \mathcal{Q}^{-1}\right)
\end{aligned}
$$

so we obtain

$$
\begin{aligned}
\frac{Z^{\prime}(s ; \mathcal{Q})}{Z(s ; \mathcal{Q})}= & {\left[\log \mathrm{m}(\mathcal{Q})+2 \log \pi+\log \mathrm{m}\left(\mathcal{Q}^{-1}\right)\right] } \\
& -\frac{\Gamma^{\prime}\left(\frac{n}{2}-s\right)}{\Gamma\left(\frac{n}{2}-s\right)}-\frac{\Gamma^{\prime}(s)}{\Gamma(s)}-\frac{Z^{\prime}\left(\frac{n}{2}-s ; \mathcal{Q}^{-1}\right)}{Z\left(\frac{n}{2}-s ; \mathcal{Q}^{-1}\right)}
\end{aligned}
$$

Therefore we have

$$
I_{3}=\int_{\frac{n}{2}-b-i T}^{\frac{n}{2}-b+i T} x^{s}\left[c_{3}-\frac{\Gamma^{\prime}\left(\frac{n}{2}-s\right)}{\Gamma\left(\frac{n}{2}-s\right)}-\frac{\Gamma^{\prime}(s)}{\Gamma(s)}-\frac{Z^{\prime}\left(\frac{n}{2}-s ; \mathcal{Q}^{-1}\right)}{Z\left(\frac{n}{2}-s ; \mathcal{Q}^{-1}\right)}\right] d s
$$

with

$$
c_{3}:=\log \mathrm{m}(\mathcal{Q})+2 \log \pi+\log \mathrm{m}\left(\mathcal{Q}^{-1}\right) .
$$

We observe that $\frac{Z^{\prime}\left(\frac{n}{2}-s ; \mathcal{Q}^{-1}\right)}{Z\left(\frac{n}{2}-s ; \mathcal{Q}^{-1}\right)}$ is again a Dirichlet series, and with another application of Lemma 5.1, we gain the representation

$$
\frac{Z^{\prime}\left(\frac{n}{2}-s ; \mathcal{Q}^{-1}\right)}{Z\left(\frac{n}{2}-s ; \mathcal{Q}^{-1}\right)}=\sum_{k=1}^{\infty} \frac{b_{k}\left(\mathcal{Q}^{-1}\right)}{l_{k}\left(\mathcal{Q}^{-1}\right)^{\left(\frac{n}{2}-s\right)}},
$$

which converges for $\operatorname{Re} s>\frac{n}{2}$. We can now divide the integral as follows:

$$
\begin{aligned}
I_{3}= & -\int_{\frac{n}{2}-b-i T}^{\frac{n}{2}-b+i T} x^{s}\left[\frac{Z^{\prime}\left(\frac{n}{2}-s ; \mathcal{Q}^{-1}\right)}{Z\left(\frac{n}{2}-s ; \mathcal{Q}^{-1}\right)}\right] d s \\
& +\int_{\frac{n}{2}-b-i T}^{\frac{n}{2}-b+i T} x^{s}\left(c_{3}-\frac{\Gamma^{\prime}\left(\frac{n}{2}-s\right)}{\Gamma\left(\frac{n}{2}-s\right)}-\frac{\Gamma^{\prime}(s)}{\Gamma(s)}\right) d s \\
= & : I_{3_{a}}+I_{3_{b}} .
\end{aligned}
$$

For the first integral we get in the area of absolute convergence

$$
\begin{aligned}
I_{3_{a}} & =-\int_{\frac{n}{2}-b-i T}^{\frac{n}{2}-b+i T} x^{s} \sum_{k=1}^{\infty} \frac{b_{k}\left(\mathcal{Q}^{-1}\right)}{l_{k}\left(\mathcal{Q}^{-1}\right)^{\left(\frac{n}{2}-s\right)}} d s \\
& =-\sum_{k=1}^{\infty} \frac{b_{k}\left(\mathcal{Q}^{-1}\right)}{l_{k}\left(\mathcal{Q}^{-1}\right)^{\frac{n}{2}}} \int_{\frac{n}{2}-b-i T}^{\frac{n}{2}-b+i T} \frac{1}{\left(x \cdot l_{k}\left(\mathcal{Q}^{-1}\right)\right)^{-s}} d s \\
& =-i x^{\frac{n}{2}-b} \sum_{k=1}^{\infty} \frac{b_{k}\left(\mathcal{Q}^{-1}\right)}{l_{k}\left(\mathcal{Q}^{-1}\right)^{b}} \int_{-T}^{T}\left(x \cdot l_{k}\left(\mathcal{Q}^{-1}\right)\right)^{i t} d t
\end{aligned}
$$


Now repeating the argument of the calculation of $I_{1}$, we see that for $x \neq$ $\frac{1}{l_{k}\left(\mathcal{Q}^{-1}\right)}$ the integral $I_{3 a}$ is $O(1)$. Taking into account the contribution for $x=\frac{1}{l_{k}\left(\mathcal{Q}^{-1}\right)}$ we obtain overall

$$
I_{3_{a}}=-i x^{\frac{n}{2}} \delta_{l_{k}\left(\mathcal{Q}^{-1}\right), \frac{1}{x}} \cdot b_{k}\left(\mathcal{Q}^{-1}\right) \cdot 2 T+O(1) .
$$

It remains to estimate $I_{3_{b}}$. Here we first apply Stirling's formula for the gamma function yielding

$$
|\Gamma(\sigma+i t)|=\sqrt{2 \pi} \cdot e^{-\frac{\pi|t|}{2}} \cdot|t|^{\sigma-\frac{1}{2}}\left(1+O\left(\frac{1}{|t|}\right)\right)
$$

and, for the logarithm,

$$
\log \Gamma(s)=\left(s-\frac{1}{2}\right) \cdot \log s-s+\frac{1}{2} \log 2 \pi+O\left(\frac{1}{|s|}\right)
$$

for $|\arg s|<\pi$ and $|s| \geq 1$. Therefore we get for the logarithmic derivative the following asymptotics:

$$
\frac{\Gamma^{\prime}(s)}{\Gamma(s)}=\log s-\frac{1}{2 s}+O\left(\frac{1}{|s|^{2}}\right)=\log s+O\left(\frac{1}{|s|}\right)
$$

see e.g. Karatsuba and Voronin [39]. To handle the branch cut of the logarithm we further separate the integral into

$$
\begin{aligned}
I_{3 b} & =\left\{\int_{\frac{n}{2}-b-i}^{\frac{n}{2}-b+i}+\int_{\frac{n}{2}-b+i}^{\frac{n}{2}-b+i T}+\int_{\frac{n}{2}-b-i T}^{\frac{n}{2}-b-i}\right\} x^{s}\left(c_{3}-\frac{\Gamma^{\prime}\left(\frac{n}{2}-s\right)}{\Gamma\left(\frac{n}{2}-s\right)}-\frac{\Gamma^{\prime}(s)}{\Gamma(s)}\right) d s \\
& =: L_{1}+L_{2}+L_{3} .
\end{aligned}
$$

Clearly we have $L_{1}=O(1)$. Next we consider

$$
L_{2}=\int_{\frac{n}{2}-b+i}^{\frac{n}{2}-b+i T} x^{s}\left[c_{3}-\log s+O\left(\frac{1}{|s|}\right)-\log \left(\frac{n}{2}-s\right)+O\left(\frac{1}{\left|\frac{n}{2}-s\right|}\right)\right] d s .
$$

We see that

$$
\begin{aligned}
& \int_{\frac{n}{2}-b+i}^{\frac{n}{2}-b+i T} x^{s}\left[c_{3}+O\left(\frac{1}{|s|}\right)+O\left(\frac{1}{\left|\frac{n}{2}-s\right|}\right)\right] d s \\
& =i x^{\frac{n}{2}-b} \int_{1}^{T} x^{i t}\left[c_{3}+O\left(\frac{1}{\left|\frac{n}{2}-b+i t\right|}\right)+O\left(\frac{1}{|b-i t|}\right)\right] d t=O(\log T) .
\end{aligned}
$$

Moreover,

$$
\begin{aligned}
& -\int_{\frac{n}{2}-b+i}^{\frac{n}{2}-b+i T} x^{s}\left[\log s+\log \left(\frac{n}{2}-s\right)\right] d s \\
& =-i x^{\frac{n}{2}-b} \int_{1}^{+T} x^{i t}\left[\log \left(\frac{n}{2}-b+i t\right)+\log (b-i t)\right] d t .
\end{aligned}
$$


Now we apply partial integration to derive

$$
\begin{aligned}
& \int_{1}^{+T} x^{i t}\left[\log \left(\frac{n}{2}-b+i t\right)+\log (b-i t)\right] d t \\
= & \left.\frac{x^{i t}}{i \log x}\left[\log \left(\frac{n}{2}-b+i t\right)+\log (b-i t)\right]\right|_{1} ^{T} \\
& -\int_{1}^{+T} \frac{x^{i t}}{\log x}\left(\frac{1}{\frac{n}{2}-b+i t}-\frac{1}{b-i t}\right) d t .
\end{aligned}
$$

Both summands on the right hand side are clearly $O(\log T)$, hence $L_{2}=$ $O(\log T)$ and the same argument yields $L_{3}=O(\log T)$. Putting the pieces together we get

$I_{3}=I_{3_{a}}+I_{3_{b}}=I_{3_{a}}+L_{1}+L_{2}+L_{3}=-i x^{\frac{n}{2}} \delta_{l_{k}\left(\mathcal{Q}^{-1}\right), \frac{1}{x}} \cdot b_{k}\left(\mathcal{Q}^{-1}\right) \cdot 2 T+O(\log T)$.

Finally

$$
I_{4}=\int_{\frac{n}{2}-b-i T}^{B-i T} x^{s} \frac{Z^{\prime}(s ; \mathcal{Q})}{Z(s ; \mathcal{Q})} d s
$$

can be estimated exactly like $I_{2}$, therefore we gain $I_{4}=O(\log T)$. This concludes the proof of the Theorem.

\subsubsection{Proof of Corollary 5.4}

Taking into account

$$
\exp (y)-1=\int_{0}^{y} \exp (t) d t \ll|y| \max \{1, \exp (y)\},
$$

we get by setting $y=\left(\beta-\frac{n}{4}\right) \log x$ with positive $x \neq 1$ the estimate

$$
\begin{aligned}
\left|x^{\left(\frac{n}{4}+i \gamma\right)}-x^{(\beta+i \gamma)}\right| & =\left|x^{i \gamma}\right|\left|x^{\frac{n}{4}}-x^{\beta}\right| \\
& =\left|x^{\beta}\left(x^{\left(\frac{n}{4}-\beta\right)}-1\right)\right| \\
& \leq x^{\beta}\left|\exp \left(\left(\frac{n}{4}-\beta\right) \log x\right)-1\right| \\
& \leq\left|\beta-\frac{n}{4}\right||\log x| \max \left\{x^{\beta}, x^{\frac{n}{4}}\right\} .
\end{aligned}
$$

In the following we use Theorem 5.3 and Weyl's criterion Theorem 2.2 to deduce uniform distribution of the imaginary parts. We proceed like in Steuding [68] and obtain

$$
\frac{1}{N(T ; \mathcal{Q})} \sum_{|\gamma|<T}\left|x^{\left(\frac{n}{4}+i \gamma\right)}-x^{(\beta+i \gamma)}\right| \leq \frac{X}{N(T ; \mathcal{Q})} \sum_{|\gamma|<T}\left|\beta-\frac{n}{4}\right|
$$


with

$$
X=\max \left\{x^{D}, 1\right\}|\log x|
$$

and $D$ as an upper bound for the real parts of the zeros. Such a positive number $D$ exists since $\zeta(s ; \mathcal{Q})$, having a Dirichlet series representation for $\operatorname{Re} s>\frac{n}{2}$, is non-vanishing in some right half plane. Using the assumption $\sum_{|\gamma|<T}\left|\beta-\frac{n}{4}\right|=o(N(T ; \mathcal{Q}))$ of Corollary 5.4 we now get

$$
\frac{1}{N(T ; \mathcal{Q})} \sum_{|\gamma|<T}\left(x^{\left(\frac{n}{4}+i \gamma\right)}-x^{(\beta+i \gamma)}\right)=o(1)
$$

where we dropped the dependency on $X$. Hence, applying the asymptotics of Theorem 5.3, that is $\sum_{|\gamma|<T} x^{\varrho}=O(T)$, yields

$$
\frac{1}{N(T ; \mathcal{Q})} \sum_{|\gamma|<T} x^{\left(\frac{n}{4}+i \gamma\right)}=o(1)
$$

Dividing by $x^{\frac{n}{4}}$ and setting $x=z^{m}$ with positive real $z \neq 1$ and $m \in \mathbb{N}$, we obtain

$$
\lim _{T \rightarrow \infty} \frac{1}{N(T ; \mathcal{Q})} \sum_{|\gamma|<T} \exp (i m \gamma \log z)=0 .
$$

Now Weyl's criterion implies the uniform distribution of the sequence $\frac{1}{2 \pi} \gamma \log z$ and finally the Epstein zeta ordinates.

\subsubsection{Proof of Corollary 5.5}

The proof proceeds very similarly to that of Corollary 5.4 and leverages Lemma 2.4. We introduce two different real parts $\beta_{\min }, \beta_{\max }$ in the exponent of $x$ and consider the term

$$
\frac{1}{N(T ; \mathcal{Q})} \sum_{\left|\gamma_{a_{k}}\right|,\left|\gamma_{b_{k}}\right|,\left|\gamma_{k}\right|<T} x^{\beta_{\min }+i \gamma_{a_{k}}}+x^{\beta_{\max }+i \gamma_{b_{k}}}-x^{\beta_{k}+i \gamma_{k}} .
$$

Separating $\left(\beta_{k}+i \gamma_{k}\right)_{k \in \mathbb{N}}$ into the two disjoint subsequences $\left(\beta_{a_{k}}+i \gamma_{a_{k}}\right)_{k \in \mathbb{N}}$ and $\left(\beta_{b_{k}}+i \gamma_{b_{k}}\right)_{k \in \mathbb{N}}$, this equals

$$
\frac{1}{N(T ; \mathcal{Q})} \sum_{\left|\gamma_{a_{k}}\right|,\left|\gamma_{b_{k}}\right|<T} x^{\beta_{m i n}+i \gamma_{a_{k}}}-x^{\beta_{a_{k}}+i \gamma_{a_{k}}}+x^{\beta_{m a x}+i \gamma_{b_{k}}}-x^{\beta_{b_{k}}+i \gamma_{b_{k}}}
$$

Analogue to (5.18) we get the estimates

$$
\left|x^{\left(\beta_{\min }+i \gamma\right)}-x^{(\beta+i \gamma)}\right| \leq\left|\beta-\beta_{\min }\right||\log x| \max \left\{x^{\beta}, x^{\beta_{\text {min }}}\right\}
$$


and

$$
\left|x^{\left(\beta_{\max }+i \gamma\right)}-x^{(\beta+i \gamma)}\right| \leq\left|\beta-\beta_{\max }\right||\log x| \max \left\{x^{\beta}, x^{\beta_{\max }}\right\}
$$

Hence we can use the common bound $X=\max \left\{x^{D}, 1\right\}|\log x|$ from (5.19). Due to our assumptions

$$
\sum_{\left|\gamma_{1 k}\right|<T}\left|\beta_{a_{k}}-\beta_{\min }\right|=o(N(T ; \mathcal{Q})) \quad \text { and } \sum_{\left|\gamma_{2 k}\right|<T}\left|\beta_{b_{k}}-\beta_{\max }\right|=o(N(T ; \mathcal{Q}))
$$

we obtain

$$
\begin{aligned}
& \frac{1}{N(T ; \mathcal{Q})} \sum_{\left|\gamma_{a_{k}}\right|,\left|\gamma_{b_{k}}\right|<T} x^{\beta_{m i n}+i \gamma_{a_{k}}}-x^{\beta_{a_{k}}+i \gamma_{a_{k}}}+x^{\beta_{\max }+i \gamma_{b_{k}}}-x^{\beta_{b_{k}}+i \gamma_{b_{k}}} \\
& \leq \frac{X}{N(T ; \mathcal{Q})}\left(\sum_{\left|\gamma_{a_{k}}\right|<T}\left|\beta_{a_{k}}-\beta_{\min }\right|+\sum_{\left|\gamma_{b_{k}}\right|<T}\left|\beta_{b_{k}}-\beta_{\max }\right|\right)=o(1) .
\end{aligned}
$$

By dropping again the dependency on $X$ we see that (5.20) tends to zero for $T \rightarrow \infty$. Using once more Theorem 5.3 we get

$$
\frac{1}{N(T ; \mathcal{Q})} \sum_{\left|\gamma_{a_{k}}\right|,\left|\gamma_{b_{k}}\right|<T} x^{\beta_{\min }+i \gamma_{a_{k}}}+x^{\beta_{\max }+i \gamma_{b_{k}}}=o(1)
$$

Now applying Lemma 2.4 concludes the proof.

\subsection{Application of the main results}

\subsubsection{The density hypothesis for binary quadratic forms}

In order to apply Corollary 5.4 we are now able to prove that Epstein zetafunctions associated with a binary quadratic form fulfill the assumptions of the density hypothesis. Hence the imaginary parts of the nontrivial zeros are uniformly distributed modulo one. That means regardless of the class number of the corresponding number field all functions based on binary quadratic forms, which are described in section 3.1, share this property. To show this we take the approach of Levinson [47] and follow closely Steuding [69]. Key will also be a mean square estimate for Dirichlet series by Müller [49].

Corollary 5.6. For binary quadratic forms, i.e. in the case $n=2$, the density hypothesis $\sum_{|\gamma|<T}\left|\beta-\frac{1}{2}\right|=o(N(T ; \mathcal{Q}))$ holds, and the ordinates of nontrivial zeros of the associated Epstein zeta-function $\zeta(s ; \mathcal{Q})$ are uniformly distributed modulo one. 
Proof: We start by stating Littlewood's Lemma relating the zeros of an analytic function $f(s)$ with a contour integral over $\log f(s)$.

Lemma 5.7 (Littlewood). Let $b<a$ and let $f(s)$ be analytic on $\mathcal{R}:=\{s \in$ $\mathbb{C}: b \leq \sigma \leq a,|t| \leq T\}$. Let $\delta \mathcal{R}$ be the border of the rectangle $\mathcal{R}$. Suppose that $f(s)$ does not vanish on the right edge $\sigma=a$ of $\mathcal{R}$. Let $\mathcal{R}^{\prime}$ be $\mathcal{R}$ minus the union of the horizontal cuts from the zeros of $f$ in $\mathcal{R}$ to the left edge of $\mathcal{R}$, and choose a single-valued branch of $\log f(s)$ in the interior of $\mathcal{R}^{\prime}$. Denote by $\nu(\sigma, T)$ the number of zeros $\sigma=\beta+i \gamma$ of $f(s)$ inside the rectangle with $\beta>\sigma$ including zeros with $\gamma=T$ but not those with $\gamma=-T$. Then

$$
\int_{\delta \mathcal{R}} \log f(s) d s=-2 \pi i \int_{b}^{a} \nu(\sigma, T) d \sigma .
$$

For the proof we refer to Titchmarsh [72], §9.9, or Littlewood's original paper [48]. We apply the lemma to $f(s)=\zeta(s ; \mathcal{Q})$ and to the rectangle $\mathcal{R}$ with vertices $a+i T, a+2 i T, b+i T, b+2 i T$. This gives

$$
\sum_{\substack{\beta>b \\ T<\gamma \leq 2 T}}(\beta-b)=\frac{-1}{2 \pi i} \int_{\delta \mathcal{R}} \log \zeta(s ; \mathcal{Q}) d s,
$$

since for the number of zeros

$$
\int_{b}^{a} \nu(\sigma, T) \mathrm{d} \sigma=\sum_{\substack{\beta>b \\ T<\gamma \leq 2 T}} \int_{b}^{\beta} \mathrm{d} \sigma=\sum_{\substack{\beta>b \\ T<\gamma \leq 2 T}}(\beta-b)
$$

holds. Here we can chose $T$ such that

$$
\min _{\gamma}|\gamma-T| \gg \frac{1}{\log T}, \quad \min _{\gamma}|\gamma-2 T| \gg \frac{1}{\log T}
$$

to ensure distance from the zeros to the boundary of $\mathcal{R}$. This is possible since the number of zeros is given by (1.3), i.e. is of the order $O(T \log T)$. Hence we can replace this $T$ later by an arbitrary $T>0$ which is allowed at the expense of an error term $O(\log T)$. Here in any interval $\left[T^{\prime}, T^{\prime}+1\right]$ for an arbitrary $T$

$$
\sum_{\substack{\beta>b \\ T^{\prime}<\gamma \leq T^{\prime}+1}}(\beta-b) \ll N\left(T^{\prime}+1 ; \mathcal{Q}\right)-N\left(T^{\prime} ; \mathcal{Q}\right) \ll \log T
$$

holds. Therefore we can find a $T$ satisfying (5.22) where the difference between the sum of interest with respect to $T^{\prime}$ and the one with respect to $T$ is only $O(\log T)$.

Continuing with the contour integral in (5.21) we now set $b \geq 1 / 2$ arbitrary and $a>1$ so that $a>\max \{b, 1+A\}$ with $A:=\sup \beta$. $A$ is finite thanks to the existence of a zero-free half plane to the right, see (5.5). We obtain from (5.21) that 


$$
\begin{aligned}
2 \pi \sum_{\substack{\beta>b \\
T<\gamma \leq 2 T}}(\beta-b)= & \int_{T}^{2 T} \log |\zeta(b+i t ; \mathcal{Q})| d t \\
& -\int_{T}^{2 T} \log |\zeta(a+i t ; \mathcal{Q})| d t \\
& -\int_{b}^{a} \arg \zeta(\sigma+i T ; \mathcal{Q}) d \sigma+\int_{b}^{a} \arg \zeta(\sigma+2 i T ; \mathcal{Q}) d \sigma \\
= & : \sum_{j=1}^{4} I_{j} .
\end{aligned}
$$

We start with $I_{2}$ which is the integral over the vertical line segment on the right edge. We recall the definitions $(1.4)$ and $(3.14)$ and set $c:=r(\mathrm{~m}(\mathcal{Q}) ; \mathcal{Q}) \neq 0$, so we can express the general Epstein zeta-function as

$$
\zeta(s ; \mathcal{Q})=c \cdot \mathrm{m}(\mathcal{Q})^{-s}\left(1+\sum_{n>\mathrm{m}(\mathcal{Q})} \frac{r(n ; \mathcal{Q})}{n^{s}} \frac{\mathrm{m}(\mathcal{Q})^{s}}{c}\right) .
$$

Hence we have

$$
\begin{aligned}
\log |\zeta(a+i t ; \mathcal{Q})|= & \log \left|c \cdot \mathrm{m}(\mathcal{Q})^{-a-i t}\right| \\
& +\log \left|1+\sum_{n>\mathrm{m}(\mathcal{Q})} \frac{r(n ; \mathcal{Q})}{c}\left(\frac{\mathrm{m}(\mathcal{Q})}{n}\right)^{a+i t}\right| .
\end{aligned}
$$

Here the absolute value of the series is less than 1 for sufficiently large $a$. Therefore we find by the Taylor expansion of the logarithm

$$
\begin{aligned}
& \int_{T}^{2 T} \log \left|1+\sum_{n>\mathrm{m}(\mathcal{Q})} \frac{r(n ; \mathcal{Q})}{c}\left(\frac{\mathrm{m}(\mathcal{Q})}{n}\right)^{a+i t}\right| d t \\
= & \left.\int_{T}^{2 T} \operatorname{Re}\left(\sum_{l=1}^{\infty} \frac{(-1)^{l+1}}{l}\left(\sum_{n>\mathrm{m}(\mathcal{Q})} \frac{r(n ; \mathcal{Q})}{c}\left(\frac{\mathrm{m}(\mathcal{Q})}{n}\right)^{a+i t}\right)\right)^{l}\right) d t \\
= & \operatorname{Re} \sum_{l=1}^{\infty} \frac{(-1)^{l+1}}{l \cdot c^{l}} \sum_{n_{1}>\mathrm{m}(\mathcal{Q})} \ldots \sum_{n_{l}>\mathrm{m}(\mathcal{Q})} r\left(n_{1} ; \mathcal{Q}\right) \cdot \ldots \cdot r\left(n_{l} ; \mathcal{Q}\right) \\
& \times\left(\frac{\mathrm{m}(\mathcal{Q})^{l}}{n_{1} \cdot \ldots \cdot n_{l}}\right)^{a} \cdot \int_{T}^{2 T}\left(\frac{\mathrm{m}(\mathcal{Q})}{n_{1} \cdot \ldots \cdot n_{l}}\right)^{i t} \\
= & B \cdot \sum_{l=1}^{\infty} \frac{1}{l}\left(\sum_{n>\mathrm{m}(\mathcal{Q})}\left(\frac{\mathrm{m}(\mathcal{Q})}{n}\right)^{a}\right)^{l} \ll 1
\end{aligned}
$$


for a constant $B$. It follows that

$$
I_{2}=\int_{T}^{2 T} \log |\zeta(a+i t ; \mathcal{Q})| d t=T \cdot \log \left|\frac{c}{m^{a}}\right|+O(1) .
$$

Considering the integral $I_{1}$ over the vertical line segment on the left we obtain first via Jensen's inequality

$$
I_{1}=\int_{T}^{2 T} \log |\zeta(b+i t ; \mathcal{Q})| d t \leq \frac{1}{2} T \cdot \log \left(\frac{1}{T} \int_{T}^{2 T}|\zeta(b+i t ; \mathcal{Q})|^{2} d t\right)
$$

Next we use a result by Müller [49]. He showed in the second part of Theorem 6.2 . that for binary quadratic forms there exists a positive constant $A(\mathcal{Q})$ such that

$$
\int_{0}^{T}\left|\zeta\left(\frac{1}{2}+i t ; \mathcal{Q}\right)\right|^{2} d t \leq A(\mathcal{Q}) \cdot T(\log T)^{2}+O(T \log T) .
$$

Adjusted for the integration limits we can find another constant $C(\mathcal{Q})$ with

$$
\int_{T}^{2 T}\left|\zeta\left(\frac{1}{2}+i t ; \mathcal{Q}\right)\right|^{2} d t \leq C(\mathcal{Q}) \cdot T(\log T)^{2}+O(T \log T)
$$

So all in all we have for a positive constant $C$

$$
I_{1}=\int_{T}^{2 T} \log \left|\zeta\left(\frac{1}{2}+i t ; \mathcal{Q}\right)\right| d t<C \cdot T \log \log T
$$

Now we can start estimating the horizontal integrals $I_{3}, I_{4}$. Suppose that $\operatorname{Re} \zeta(\sigma+i T ; \mathcal{Q})$ has $N$ zeros for $b \leq \sigma \leq a$. We can divide the interval $[b, a]$ into at most $N+1$ subintervals in each of which $\operatorname{Re} \zeta(\sigma+i T ; \mathcal{Q})$ shows constant sign. Then

$$
|\arg \zeta(\sigma+i T ; \mathcal{Q})| \leq(N+1) \pi
$$

To estimate $N$ let

$$
g(z)=\frac{1}{2}(\zeta(z+i T ; \mathcal{Q})+\overline{\zeta(\bar{z}+i T ; \mathcal{Q})}) .
$$

Then we have $g(\sigma)=\operatorname{Re} \zeta(\sigma+i T ; \mathcal{Q})$. Let now $R=a-b$ and choose $T$ so large that $T>2 R$. We obtain $\operatorname{Im}(z+i T)>0$ for $|z-a|<T$. Thus $\zeta(z+i T ; \mathcal{Q})$, and hence $g(z)$ is analytic for $|z-a|<T$. Let $n(r)$ denote the number of zeros of $g(z)$ in $|z-a| \leq r$. Obviously, we have

$$
\int_{0}^{2 R} \frac{n(r)}{r} \mathrm{~d} r \geq n(R) \int_{R}^{2 R} \frac{\mathrm{d} r}{r}=n(R) \log 2 .
$$

With Jensen's formula (see for example Titchmarsh [72], §3.61), 


$$
\int_{0}^{2 R} \frac{n(r)}{r} \mathrm{~d} r=\frac{1}{2 \pi} \int_{0}^{2 \pi} \log \left|g\left(a+2 R e^{i \theta}\right)\right| \mathrm{d} \theta-\log |g(a)|
$$

we deduce

$$
n(R) \leq \frac{1}{2 \pi \log 2} \int_{0}^{2 \pi} \log \left|g\left(a+2 R e^{i \theta}\right)\right| \mathrm{d} \theta-\frac{\log |g(a)|}{\log 2} .
$$

By (5.23) it follows that $\log |g(a)|$ is bounded. By (5.11), in any vertical strip of bounded width,

$$
\zeta(s ; \mathcal{Q}) \ll|t|^{B}
$$

as $|t| \rightarrow \infty$ with a certain positive constant $B$. Obviously, the same estimate holds for $g(z)$. Thus, the integral above is $\ll \log T$, and $n(R) \ll \log T$. Since the interval $(b, a)$ is contained in the disc $|z-a| \leq R$, the number $N$ is less than or equal to $n(R)$. Therefore, with (5.24), we get

$$
\left|I_{3}\right| \leq \int_{b}^{a}|\arg \zeta(\sigma+i T ; \mathcal{Q})| \mathrm{d} \sigma \ll \log T .
$$

Obviously, $I_{4}$ can be bounded in the same way.

Considering all estimates this yields

$$
\sum_{\substack{\beta>\frac{1}{2} \\ T<\gamma \leq 2 T}}\left(\beta-\frac{1}{2}\right) \ll T \cdot \log \log T=o(N(T ; \mathcal{Q})) .
$$

Due to the functional equation (1.2) we now observe that the same estimate also applies to the zeros left of the critical line. Every zero $\varrho=\beta+i \gamma$ of $\zeta(s ; \mathcal{Q})$ with real part less than $1 / 2$ corresponds to a zero of $\zeta\left(s ; \mathcal{Q}^{-1}\right)$ with real part greater than $1 / 2$ and vice-versa. Also for binary quadratic forms $\zeta(s ; \mathcal{Q})$ and $\zeta\left(s ; \mathcal{Q}^{-1}\right)$ are equal up to a constant factor, as mentioned in section 3.5.1. Hence

$$
\sum_{\substack{\beta<\frac{1}{2} \\ T<\gamma \leq 2 T}}\left|\beta-\frac{1}{2}\right|=\sum_{\substack{\beta^{*}>\frac{1}{2} \\ T<-\gamma^{*} \leq 2 T}}\left(\beta^{*}-\frac{1}{2}\right) \ll T \cdot \log \log T,
$$

where $\beta^{*}+i \gamma^{*}=\frac{n}{2}-(\beta+i \gamma)$ for $n=2$. This finishes the proof of Corollary 5.6 .

\subsubsection{Application to Epstein zeta-functions for $n=4$ and $n=8$}

Corollary 5.5 can be applied to specific examples of Epstein zeta-functions where the associated quadratic form carries more than two variables to establish uniform distribution modulo one of the zero ordinates. It provides an alternative proof for instance for the functions introduced in (3.11) and (3.12). We recall their structure. For 


$$
\zeta\left(s ; \mathbf{I}_{4}\right)=8\left(1-2^{2-s}\right) \zeta(s) \zeta(s-1)
$$

we can divide the nontrivial zeros into two disjoint sets

$$
\left\{\varrho_{a_{k}} \in \mathbb{C} \mid \zeta\left(\varrho_{a_{k}}\right)=0 \quad \text { or } \quad 1-2^{2-\varrho_{a_{k}}}=0\right\}_{a_{k} \in \mathbb{N}}
$$

and

$$
\left\{\varrho_{b_{k}} \in \mathbb{C} \mid \zeta\left(\varrho_{b_{k}}-1\right)=0\right\}_{b_{k} \in \mathbb{N}}
$$

If we order the union of these two sets by the absolute values of their imaginary parts we obtain two disjoint subsets $A=\left\{a_{1}, a_{2}, \ldots\right\}$ and $B=\left\{b_{1}, b_{2}, \ldots\right\}$ of $\mathbb{N}$ with $A \cup B=\mathbb{N}$. Hence $\left(\beta_{k}+i \gamma_{k}\right)_{k \in \mathbb{N}}$ can be separated into two subsequences $\left(\beta_{a_{k}}+i \gamma_{a_{k}}\right)_{k \in \mathbb{N}},\left(\beta_{b_{k}}+i \gamma_{b_{k}}\right)_{k \in \mathbb{N}}$. We set $\beta_{\text {min }}=1 / 2$ and $\beta_{\max }=3 / 2$ and observe that the solutions of $1-2^{2-\varrho_{a}}=0$ are only of the magnitude of $C T$ for a constant $C$ with respect to the imaginary axis. Therefore due to Levinson [47], see section 2.6 for the Riemann zeta-function, the density assumptions are fulfilled, i.e.

$$
\sum_{\left|\gamma_{a_{k}}\right|<T}\left|\beta_{a_{k}}-\frac{1}{2}\right|=O(T)+O\left(T(\log \log T)^{2}\right)=o(N(T ; \mathcal{Q}))
$$

and

$$
\sum_{\left|\gamma_{b_{k}}\right|<T}\left|\beta_{b_{k}}-\frac{3}{2}\right|=O\left(T(\log \log T)^{2}\right)=o(N(T ; \mathcal{Q})),
$$

so the zero ordinates are uniformly distributed. The same reasoning applies to

$$
\zeta\left(s ; \mathcal{S}_{8}\right)=240 \cdot 2^{-s} \zeta(s) \zeta(s-3) .
$$

with $\beta_{\min }=1 / 2$ and $\beta_{\max }=7 / 2$. Here, of course, there are no other zeros than those implied by the Riemann zeta-function.

As indicated these are only alternative proofs. We know already from the properties of the Riemann zeta-function and Theorem 2.3 that the zero ordinates are uniformly distributed modulo one. Nevertheless in general it is possible that examples exist where two clustering lines can be established to apply Corollary 5.5 without having a simple product form of $\zeta(s ; \mathcal{Q})$.

Finally we remark that Corollary 5.5 is valid also for all other zeta-functions where an equivalent of Landau's theorem can be established, hence for example for functions discussed in Chapter 4. 


\subsection{Recursion formula for the logarithmic derivative}

In this section we will derive a recursive representation for the logarithmic derivative $\frac{Z^{\prime}(s ; \mathcal{Q})}{Z(s ; \mathcal{Q})}$. We will also show that it agrees with Lemma 5.1. We will split $\frac{Z^{\prime}(s ; \mathcal{Q})}{Z(s ; \mathcal{Q})}$ into two summands depending on if the $l_{n}(\mathcal{Q})$ as defined in $(5.7)$ consists of only one factor $(h=1)$ or is the product of at least two factors $(h \geq 2)$. The first summand of this representation will equal $Z^{\prime}(s ; \mathcal{Q})$ and for the second summand we will define quantities $c_{k}$ and $q_{k}$ recursively. The $q_{k}$ will run through all possible products of the form (5.7) with at least two factors. The $c_{k}$ will be the corresponding coefficients. In particular we claim

Lemma 5.8. For the logarithmic derivative of $Z(s ; \mathcal{Q})$ as defined in previous sections we have the following recursive relation:

$$
\frac{Z^{\prime}(s ; \mathcal{Q})}{Z(s ; \mathcal{Q})}=\sum_{n>\mathrm{m}(\mathcal{Q})} a(n) \log \left(\frac{\mathrm{m}(\mathcal{Q})}{n}\right)\left(\frac{\mathrm{m}(\mathcal{Q})}{n}\right)^{s}+\sum_{k=0}^{\infty} c_{k} \cdot q_{k}{ }^{-s}
$$

For $k \geq 0$ the $q_{k}$ are defined in increasing order as

$$
\begin{aligned}
q_{0} & =\frac{l_{1}^{2}}{\mathrm{~m}(\mathcal{Q})^{2}} \\
q_{k} & =\min _{i \leq k-1}\left\{\left(q_{i} \cdot \frac{l_{2}}{\mathrm{~m}(\mathcal{Q})}\right),\left(\frac{l_{3}}{\mathrm{~m}(\mathcal{Q})} \cdot \frac{l_{4}}{\mathrm{~m}(\mathcal{Q})}\right)\right\}
\end{aligned}
$$

with $q_{k}>q_{k-1}, l_{1}=\mathrm{m}(\mathcal{Q})+1$ and in general all numbers $l_{i} \in \mathbb{N}$ are greater than $\mathrm{m}(\mathcal{Q})$. For the corresponding $c_{k}$ 's, we have, with $i_{j}<k$ and $l_{2_{j}}>\mathrm{m}(\mathcal{Q})$,

$c_{k}= \begin{cases}-a\left(l_{2_{1}}\right) c_{i_{1}}-\ldots-a\left(l_{2_{\alpha}}\right) c_{i_{\alpha}} & \text { if } q_{k}=q_{i_{1}} \frac{l_{2_{1}}}{\mathrm{~m}(\mathcal{Q})}=\ldots=q_{i_{\alpha}} \frac{l_{2_{\alpha}}}{\mathrm{m}(\mathcal{Q})} \\ -a\left(l_{3}\right)^{2} \log \frac{\mathrm{m}(\mathcal{Q})}{l_{3}} & \text { if } q_{k}=\frac{l_{3}}{\mathrm{~m}(\mathcal{Q})} \cdot \frac{l_{4}}{\mathrm{~m}(\mathcal{Q})} \text { and } l_{3}=l_{4} \\ -a\left(l_{4}\right) a\left(l_{3}\right) \log \frac{\mathrm{m}(\mathcal{Q})}{l_{3}}-a\left(l_{3}\right) a\left(l_{4}\right) \log \frac{\mathrm{m}(\mathcal{Q})}{l_{4}} & \text { if } q_{k}=\frac{l_{3}}{\mathrm{~m}(\mathcal{Q})} \cdot \frac{l_{4}}{\mathrm{~m}(\mathcal{Q})} \text { and } l_{3} \neq l_{4} .\end{cases}$

Proof: First we note that obviously $\frac{Z^{\prime}(s ; \mathcal{Q})}{Z(s ; \mathcal{Q})} \times Z(s ; \mathcal{Q})=Z^{\prime}(s ; \mathcal{Q})$, and from this product representation we can derive the coefficients recursively by comparison of the series. Now the first sum of $(5.25)$ is equal to $Z^{\prime}(s ; \mathcal{Q})$ and stems from the fact that $Z(s ; \mathcal{Q})$ starts with the constant term 1 . In the second term, $q_{0}$ is the first base, which needs to occur since the lowest base of $\frac{Z^{\prime}(s ; \mathcal{Q})}{Z(s ; \mathcal{Q})}$ is $\left(\frac{l_{1}}{\mathrm{~m}(\mathcal{Q})}\right)^{-s}$. Hence we need to define $c_{0}$ as above to cancel out $a\left(l_{1}\right) \log \frac{\mathrm{m}(\mathcal{Q})}{l_{1}} \times a\left(l_{1}\right)$.

Now we consider the $q_{k}$ defined in increasing order. The construction of the 
$q_{k}$ ensures to represent all possible products with at least two factors, which are clearly of the form of the $l_{n}(\mathcal{Q})$; see (5.7). In each step of the recursion, either the next larger $q_{k}$ is a new product $\frac{l_{3}}{\mathrm{~m}(\mathcal{Q})} \cdot \frac{l_{4}}{\mathrm{~m}(\mathcal{Q})}$, or it is a product of an already existing $q_{i}$ with one new factor $\frac{l_{4}}{\mathrm{~m}(\mathcal{Q})}$. The $c_{k}$ are defined such that by multiplication with $Z(s ; \mathcal{Q})$ the coefficient of the lowest base is cancelled out. This means that if $q_{k}$ is of the form $q_{i} \cdot \frac{l_{2}}{\mathrm{~m}(\mathcal{Q})}$, then we have $c_{k}=-a\left(l_{2_{1}}\right) c_{i_{1}}-\ldots-a\left(l_{2_{\alpha}}\right) c_{i_{\alpha}}$ since the $q_{k}$ can equal several different products of this form and we need to cancel out all the contributions. If we have new products of the form $q_{k}=\frac{l_{3}}{\mathrm{~m}(\mathcal{Q})} \cdot \frac{l_{4}}{\mathrm{~m}(\mathcal{Q})}$, then we again achieve the cancellation by the previous definitions. Here we note that the last case differentiation occurs if either $l_{3}=l_{4}$ where there is only one term to be cancelled or if $l_{3} \neq l_{4}$ where the two symmetric coefficients need to be wiped out.

To see that both representations from Lemma 5.1 and Lemma 5.8 coincide we first note that the number of weights $\frac{l_{i}}{\mathrm{~m}(\mathcal{Q})}$ in the $c_{k}$ grows according to the multinomial coefficient which is the essential part of the $b_{n}(\mathcal{Q})$ defined in (5.8). Further the recursive identity $c_{k}=-a\left(l_{2_{1}}\right) c_{i_{1}}-\ldots-a\left(l_{2_{\alpha}}\right) c_{i_{\alpha}}$ corresponds with that of the multinomial coefficient

$$
\left(\begin{array}{c}
\nu \\
k_{1} \ldots k_{r}
\end{array}\right)=\sum_{i=1}^{r}\left(\begin{array}{c}
\nu-1 \\
k_{1} \ldots k_{i-1}, k_{i}-1, k_{i+1} \ldots k_{r}
\end{array}\right) .
$$

Finally the factors $a\left(\mathrm{~m}(\mathcal{Q})+k_{i}\right) \log \left(\frac{\mathrm{m}(\mathcal{Q})}{\mathrm{m}(\mathcal{Q})+k_{i}}\right)$ in $b_{n}(\mathcal{Q})$ arise from the multiplication with $Z^{\prime}(s ; \mathcal{Q})$.

\subsection{Open questions}

The central natural open question remains: Does uniform distribution modulo one of the zero ordinates hold for all Epstein zeta-functions? At least no good argument against it has been found during the work of this thesis. We have seen in this chapter that for more than two clustering lines of zeros the argumentation via a Landau type theorem doesn't suffice and that there are definitely many Epstein zeta-functions with a more complex zero distribution, see Chapter 3 . On the other hand the author is not aware of a zeta-function where the ordinates of the nontrivial zeros $\varrho=\beta+i \gamma$ share a different distribution property modulo one.

A different approach to gain uniform distribution results had been suggested by Elliott [17]. His reasoning relies on a careful analysis of the argument of the Riemann zeta-function. However, its not clear if this method can be applied to the general case.

Another interesting open question fitting into this thesis is: For which type of zeta-function can we establish a Landau type theorem, or, at least, a bound of the form $\sum_{0<\gamma \leq T} x^{\varrho}=O(T)$ ? 
We mentioned a couple of examples throughout previous chapters. In order to use the same technique of contour integration as we did, it looks reasonable to demand that the zeta-function obeys a functional equation, the PhragménLindelöf principle and comes along with a zero-free half plane. 


\title{
Discrepancy estimate for zero ordinates of Epstein zeta-functions
}

\author{
"A mathematician is a device for turning coffee into theorems."
}

Paul Erdös (1913-1996)

In this last chapter we shall investigate into the discrepancy of the sequence of zero ordinates of Epstein zeta-functions. We first will give basic definitions and recall some results valid for the Riemann zeta-function. For a discrepancy estimate we need a uniform version of Theorem 5.3. To prove this we will use the Theorem of Erdös and Turán and ideas of Rehberg [56], as well as Baluyot and Gonek [3].

\subsection{Preliminaries}

So far we studied uniform distribution modulo one purely from a qualitative point of view. One measure to describe the quantitative distribution property of a sequence is the so-called discrepancy. It was first introduced by Bergström [5] and van der Corput and Pisot [75]. If $x_{1}, \ldots, x_{N}$ is a finite sequence of real numbers, then the number

$$
\begin{aligned}
D_{N} & =D_{N}\left(x_{1}, \ldots, x_{N}\right) \\
& =\sup _{0 \leq \alpha<\beta \leq 1}\left|\frac{\#\left\{1 \leq n \leq N:\left\{x_{n}\right\} \in[\alpha, \beta)\right\}}{N}-(\beta-\alpha)\right|
\end{aligned}
$$

is called the discrepancy of the sequence. If the sequence $\left(x_{n}\right)_{n \geq 1}$ is infinite or contains at least $N$ terms, $D_{N}$ is defined to be the discrepancy of the first $N$ terms of the sequence. The discrepancy measures the deviation between the distribution of the sequence and uniform distribution modulo one. A sequence is uniformly distributed modulo one if, and only if, its discrepancy tends to zero for $N \rightarrow \infty$. 
We briefly want to examine what bounds this quantity has to obey. The convergence cannot be arbitrarily fast, for every sequence of $N$ numbers we have

$$
\frac{1}{N} \leq D_{N} \leq 1
$$

According to a result of van Aardenne-Ehrenfest [74] in 1945 every sequence satisfies for some positive constant $k$ the lower bound

$$
D_{N} \geq \frac{k \cdot \log \log \log N}{N}
$$

for infinitely many $N$, but not for all. This has been improved by Roth [58] to

$$
D_{N} \geq \frac{\sqrt{\log N}}{16 N}
$$

for infinitely many $N$ and further by Schmidt [60] to

$$
D_{N} \geq \frac{1}{66 \log 4} \frac{\log N}{N} .
$$

We will concentrate on upper bounds in the sequel though. A useful method to estimate the discrepancy is by means of exponential sums appearing in Weyl's criterion (2.2). This allows techniques from analytic number theory to be applied for the estimates. One important result in this direction, which we will leverage, is the Theorem of Erdös and Turán [19]: Let $x_{1}, \ldots, x_{N}$ be a finite sequence of real numbers and $m$ any positive integer. Then

$$
D_{N} \leq \frac{6}{m+1}+\frac{4}{\pi} \sum_{h=1}^{m}\left(\frac{1}{h}-\frac{1}{m+1}\right)\left|\frac{1}{N} \sum_{n=1}^{N} e^{2 \pi i h x_{n}}\right| .
$$

In particular we will use the following simplified version: There exists an absolute constant $C$ such that

$$
D_{N} \leq C \cdot\left(\frac{1}{m}+\sum_{h=1}^{m} \frac{1}{h}\left|\frac{1}{N} \sum_{n=1}^{N} e^{2 \pi i h x_{n}}\right|\right),
$$

see for example equation (2.42) in Kuipers and Niederreiter [42]. Here it is important that the parameter $m$ can be chosen freely to optimize the estimate.

We will now recall some results about the discrepancy of the Riemann zeta ordinates which are uniformly distributed modulo one. In 1990 Hlawka [36] was the first to prove the following: Let $z>1$ be a real number and $D_{T}$ the discrepancy of the sequence $\left(\frac{\log z}{2 \pi} \gamma\right)_{\gamma}$. Then, for $T \rightarrow \infty$

$$
D_{T}=O\left(\frac{\log z}{\log \log T}\right)
$$


and by assuming the Riemann hypothesis

$$
D_{T}=O\left(\frac{\log z}{\log T}\right) .
$$

In the proof Hlawka used the Theorem of Erdös and Turán (6.1) and an improved error term from Landau's theorem (1.6) determining the $x$-dependency. He was able to replace $O(\log T)$ with $O\left(x^{2} \log T\right)$ which enabled him to choose an optimal parameter $m$ in (6.1). In 1993 Fujii [26] obtained an improved discrepancy estimate, he replaced (6.2) with

$$
D_{T}=O\left(\frac{\log \log T}{\log T}\right)
$$

where the implied constant depends on $z$. For further refinements see also Fujii [27].

We turn our attention back to the general case of Epstein zeta-functions though. To ensure uniform distribution modulo one of the zero ordinates we needed the average density hypothesis. Hence this assumption is still necessary to explore the discrepancy, and we additionally require a certain speed of convergence. We will therefore demand

$$
\sum_{|\gamma|<T}\left|\beta-\frac{n}{4}\right|=O\left(\frac{T \log T}{\log \log T}\right)
$$

for the real parts $\beta$ of $\zeta(s ; \mathcal{Q})$. This, as recalled in $(2.6)$, was proven by Levinson [47] for the Riemann zeta-function. It is a natural assumption to make, since this result generalizes to a broader class of zeta-functions, see also Levinson [47].

Finally in this section we provide an estimate of the coefficients $b_{n}(\mathcal{Q})$ occurring in the general representation of the logarithmic derivative of $Z(s ; \mathcal{Q})$, defined in Lemma 5.1. This will be used to derive the statement about the discrepancy in the following sections.

Lemma 6.1. Let $\frac{Z^{\prime}(s ; \mathcal{Q})}{Z(s ; \mathcal{Q})}=\sum_{n=1}^{\infty} \frac{b_{n}(\mathcal{Q})}{l_{n}(\mathcal{Q})^{s}}$. There is a constant $B>0$ such that for all $n \in \mathbb{N}$ we have

$$
b_{n}(\mathcal{Q})=O\left(l_{n}(\mathcal{Q})^{B}\right)
$$

Proof: $\frac{Z^{\prime}(s ; \mathcal{Q})}{Z(s ; \mathcal{Q})}=\sum_{n=1}^{\infty} \frac{b_{n}(\mathcal{Q})}{l_{n}(\mathcal{Q})^{s}}$ converges absolutely for $\sigma>B$ with $B>0$ the constant defined in (5.10). It is known, see for instance Hardy and Riesz [33], that the abscissa of convergence satisfies

$$
\sigma=\limsup _{N \rightarrow \infty} \frac{\log \sum_{n=1}^{N}\left|b_{n}(\mathcal{Q})\right|}{\log l_{n}(\mathcal{Q})},
$$

respectively 


$$
\sigma=\limsup _{N \rightarrow \infty} \frac{\log \sum_{n=N+1}^{\infty}\left|b_{n}(\mathcal{Q})\right|}{\log l_{n}(\mathcal{Q})}
$$

according to whether $\sum_{n=1}^{N}\left|b_{n}(\mathcal{Q})\right|$ is divergent or not. That implies in the latter case $l_{n}(\mathcal{Q})^{B}+\epsilon \geq \sum_{n=N+1}^{\infty}\left|b_{n}(\mathcal{Q})\right|$ for all $\epsilon>0$ and sufficiently large $N \in \mathbb{N}$. Therefore

$$
\left|b_{N+1}(\mathcal{Q})\right|=\sum_{n=N+1}^{\infty}\left|b_{n}(\mathcal{Q})\right|-\sum_{n=N+2}^{\infty}\left|b_{n}(\mathcal{Q})\right| \leq l_{n}(\mathcal{Q})^{B} .
$$

The case when $\sum_{n=N+1}^{\infty}\left|b_{n}(\mathcal{Q})\right|$ is divergent can be treated in the same way which implies $b_{n}(\mathcal{Q})=O\left(l_{n}(\mathcal{Q})^{B}\right)$.

\subsection{Uniform version of Theorem 5.3}

Our aim is now to derive a uniform version of Theorem 5.3 by exploring the dependency on the parameter $x$. For this we need to recall the ordered set $S$ of elements

$$
l_{n}(\mathcal{Q})=\prod_{\nu=1}^{h} \frac{\mathrm{m}(\mathcal{Q})+k_{\nu}}{\mathrm{m}(\mathcal{Q})}
$$

with $h \geq 0, k_{\nu} \in \mathbb{N}$, see (5.7). For $x \in \mathbb{R}^{+} \backslash\{1\}$ we define

$$
\langle x\rangle=\left\{\begin{array}{llll}
\text { nearest distance to a } & l_{n}(\mathcal{Q}) & \text { if } & x>1 \\
\text { nearest distance to a } & \frac{1}{l_{n}\left(\mathcal{Q}^{-1}\right)} & \text { if } & x<1 .
\end{array}\right.
$$

Looking at the dependency on the parameter $x$ we will prove the following variant of Theorem 5.3:

Theorem 6.2. Let $x \in \mathbb{R}^{+} \backslash\{1\}$ and $B$ as defined in (5.10), $b>B$. As $T \rightarrow \infty$ there exists a constant $\tilde{B}>0$ such that

$$
\begin{aligned}
\sum_{|\gamma|<T} x^{\varrho}= & \frac{T}{\pi}\left(\delta_{l_{k}(\mathcal{Q}), x} \cdot b_{k}(\mathcal{Q})-x^{\frac{n}{2}} \delta_{l_{k}\left(\mathcal{Q}^{-1}\right), \frac{1}{x}} \cdot b_{k}\left(\mathcal{Q}^{-1}\right)\right) \\
& +O\left(x^{B} \log T\right) \mathbf{1}_{\{x>1\}}+O\left(x^{\frac{n}{2}-b} \log T\right) \mathbf{1}_{\{0<x<1\}} \\
& +O\left(x^{\tilde{B}} \cdot \min \left(T, \frac{x}{\langle x\rangle}\right)\right) \mathbf{1}_{\{x>1\}} \\
& +O\left(x^{-\tilde{B}} \cdot \min \left(T, \frac{x}{\langle x\rangle}\right)\right) \mathbf{1}_{\{0<x<1\}}
\end{aligned}
$$

for all Epstein zeta-functions. 
Proof: We use the same setup as for Theorem 5.3 and integrate via the path of the counterclockwise oriented closed rectangle $\partial \mathcal{R}$ with points $B-i T$, $B+i T, \frac{n}{2}-b+i T$ and $\frac{n}{2}-b-i T$. All nontrivial zeros of $\zeta(s ; \mathcal{Q})$ lie inside $\frac{n}{2}-b<\operatorname{Re} s<B$ and we consider, as in (5.13),

$$
\sum_{|\gamma|<T} x^{\varrho}=\frac{1}{2 \pi i} \int_{\partial \mathcal{R}} x^{s} \frac{Z^{\prime}(s ; \mathcal{Q})}{Z(s ; \mathcal{Q})} d s+O(1) .
$$

Again like in (5.14) we split the integral into four parts

$$
\begin{aligned}
\int_{\partial \mathcal{R}} x^{s} \frac{Z^{\prime}(s ; \mathcal{Q})}{Z(s ; \mathcal{Q})} d s= & \left\{\int_{B-i T}^{B+i T}+\int_{B+i T}^{\frac{n}{2}-b+i T}+\right. \\
& \left.+\int_{\frac{n}{2}-b+i T}^{\frac{n}{2}-b-i T}+\int_{\frac{n}{2}-b-i T}^{B-i T}\right\} x^{s} \frac{Z^{\prime}(s ; \mathcal{Q})}{Z(s ; \mathcal{Q})} d s \\
=: & \sum_{j=1}^{4} I_{j} .
\end{aligned}
$$

First we show that

$$
\begin{aligned}
I_{1} & =i \sum_{n=1}^{\infty} b_{n}(\mathcal{Q})\left(\frac{x}{l_{n}(\mathcal{Q})}\right)^{B} \int_{-T}^{+T}\left(\frac{x}{l_{n}(\mathcal{Q})}\right)^{i t} d t \\
& =2 i \cdot \delta_{l_{k}(\mathcal{Q}), x} \cdot b_{k}(\mathcal{Q}) \cdot T+O\left(x^{B} \sum_{n=1}^{\infty} \frac{\left|b_{n}(\mathcal{Q})\right|}{l_{n}(\mathcal{Q})^{B}} \cdot \min \left\{T, \frac{1}{\left|\log \left(\frac{x}{l_{n}(\mathcal{Q})}\right)\right|}\right\}\right) .
\end{aligned}
$$

Here the first term on the right stems from the case $x=l_{n}(\mathcal{Q})$. The error term arises for $x \neq l_{n}(\mathcal{Q})$ where we get

$$
\int_{-T}^{+T}\left(\frac{x}{l_{n}(\mathcal{Q})}\right)^{i t} d t=i \cdot \frac{1}{\log \left(\frac{x}{l_{n}(\mathcal{Q})}\right)}\left(e^{-\log \left(\frac{x}{l_{n}(\mathcal{Q})}\right) i T}-e^{\log \left(\frac{x}{l_{n}(\mathcal{Q})}\right) i T}\right)
$$

which cannot grow larger than $O(T)$. This can be seen by inserting the Taylor approximation of the exponential function. Now to estimate the sum in the error term we note that for $l_{n}(\mathcal{Q}) \leq \frac{1}{2} x$ or $l_{n}(\mathcal{Q}) \geq 2 x$ we have

$$
\left|\log \left(\frac{x}{l_{n}(\mathcal{Q})}\right)\right| \gg 1 \text {. }
$$

Thus, due to the absolute convergence of $\sum_{n=1}^{\infty} \frac{b_{n}(\mathcal{Q})}{l_{n}(\mathcal{Q})^{s}}$, this part is 


$$
\ll \sum_{n=1}^{\infty} \frac{\left|b_{n}(\mathcal{Q})\right|}{l_{n}(\mathcal{Q})^{B}} \cdot x^{B} \ll x^{B} .
$$

On the other hand the part with $\frac{1}{2} x<l_{n}(\mathcal{Q})<x$ is

$$
\ll \sum_{\frac{1}{2} x<l_{n}(\mathcal{Q})<x}\left|b_{n}(\mathcal{Q})\right| \cdot \min \left\{T, \frac{1}{\left|\log \left(\frac{x}{l_{n}(\mathcal{Q})}\right)\right|}\right\} .
$$

Since $l_{n}(\mathcal{Q}) \rightarrow \infty$ there are only finitely many $l_{n}(\mathcal{Q})$ below any fixed $x$. Also there exists an $\alpha \in \mathbb{N}$ such that $l_{\alpha}(\mathcal{Q})<x<l_{\alpha+1}(\mathcal{Q})$. Thus, since

$$
\log \left(\frac{x}{l_{n}(\mathcal{Q})}\right)=-\log \left(1-\frac{x-l_{n}(\mathcal{Q})}{x}\right)>\frac{l_{\alpha}(\mathcal{Q})-l_{n}(\mathcal{Q})}{x}
$$

we get by applying Lemma 6.1

$$
\begin{aligned}
\sum_{\frac{1}{2} x<l_{n}(\mathcal{Q})<x}\left|b_{n}(\mathcal{Q})\right| & \cdot \min \left\{T, \frac{1}{\left|\log \left(\frac{x}{l_{n}(\mathcal{Q})}\right)\right|}\right\} \\
& \ll \#\left\{n \in \mathbb{N}: l_{n}(\mathcal{Q})<x\right\} \cdot l_{\alpha}(\mathcal{Q})^{B} \cdot \min \left\{T, \frac{x}{x-l_{\alpha}(\mathcal{Q})}\right\} \\
& \ll \#\left\{n \in \mathbb{N}: l_{n}(\mathcal{Q})<x\right\} \cdot x^{B} \cdot \min \left\{T, \frac{x}{\langle x\rangle}\right\} .
\end{aligned}
$$

It remains to consider the number of elements in $S$ with $l_{n}(\mathcal{Q})<x$. We use a crude estimate sufficient to bound this number with a power of $x$. The denominator of a number $l_{n}(\mathcal{Q})$, see definition (5.7), is a power of $\mathrm{m}(\mathcal{Q})$. From $l_{n}(\mathcal{Q})<x$ it follows that the largest possible power $h_{1} \in \mathbb{R}$ occurring in the representation of $l_{n}(\mathcal{Q})$ fulfills

$$
\left(\frac{\mathrm{m}(\mathcal{Q})+1}{\mathrm{~m}(\mathcal{Q})}\right)^{h_{1}}<x
$$

hence

$$
h_{1}<\frac{\log x}{\log \left(\frac{\mathrm{m}(\mathcal{Q})+1}{\mathrm{~m}(\mathcal{Q})}\right)} .
$$

Further to any denominator $\mathrm{m}(\mathcal{Q})^{h}$ there exist at most $x \cdot \mathrm{m}(\mathcal{Q})^{h}$ numbers less than $x$. Therefore we can bound the number of elements in $\left\{n \in \mathbb{N}: l_{n}(\mathcal{Q})<\right.$ $x\}$ by $x \cdot \mathrm{m}(\mathcal{Q})^{h_{1}} \cdot h_{1}$. Defining

$$
D_{1}:=\left[\frac{\log \mathrm{m}(\mathcal{Q})}{\log \left(\frac{\mathrm{m}(\mathcal{Q})+1}{\mathrm{~m}(\mathcal{Q})}\right)}\right]+2
$$


we arrive at

$$
\#\left\{n \in \mathbb{N}: l_{n}(\mathcal{Q})<x\right\} \ll x^{D_{1}} .
$$

An analogous argument holds for the part $x<l_{n}(\mathcal{Q})<2 x$. Putting pieces together we see that

$$
I_{1}=2 i \cdot \delta_{l_{k}(\mathcal{Q}), x} \cdot b_{k}(\mathcal{Q}) \cdot T+O\left(x^{B} \cdot x^{D_{1}} \cdot \min \left\{T, \frac{x}{\langle x\rangle}\right\}\right) .
$$

Looking at $I_{2}$ we follow closely the arguments of Chapter 5 , in particular (5.16), to arrive at

$$
\begin{aligned}
I_{2} & =\int_{\frac{n}{2}-b+i T}^{B+i T} x^{s} \frac{Z^{\prime}(s ; \mathcal{Q})}{Z(s ; \mathcal{Q})} d s \ll \int_{\frac{n}{2}-b}^{B} x^{\sigma}\left|\frac{Z^{\prime}(\sigma+i T ; \mathcal{Q})}{Z(\sigma+i T ; \mathcal{Q})}\right| d \sigma \\
& \ll(\log T) x^{B} \mathbf{1}_{\{x>1\}}+(\log T) x^{\frac{n}{2}-b} \mathbf{1}_{\{0<x<1\}} \\
& =O\left(x^{B} \log T\right) \mathbf{1}_{\{x>1\}}+O\left(x^{\frac{n}{2}-b} \log T\right) \mathbf{1}_{\{0<x<1\}} .
\end{aligned}
$$

Likewise we get

$$
I_{4}=\int_{\frac{n}{2}-b-i T}^{B-i T} x^{s} \frac{Z^{\prime}(s ; \mathcal{Q})}{Z(s ; \mathcal{Q})} d s=O\left(x^{B} \log T\right) \mathbf{1}_{\{x>1\}}+O\left(x^{\frac{n}{2}-b} \log T\right) \mathbf{1}_{\{0<x<1\}} .
$$

Now we consider $I_{3}$ and decompose as above, see (5.17) onwards, into

$$
\begin{aligned}
I_{3}= & -\int_{\frac{n}{2}-b-i T}^{\frac{n}{2}-b+i T} x^{s}\left[\frac{Z^{\prime}\left(\frac{n}{2}-s ; \mathcal{Q}^{-1}\right)}{Z\left(\frac{n}{2}-s ; \mathcal{Q}^{-1}\right)}\right] d s \\
& +\int_{\frac{n}{2}-b-i T}^{\frac{n}{2}-b+i T} x^{s}\left(c_{3}-\frac{\Gamma^{\prime}\left(\frac{n}{2}-s\right)}{\Gamma\left(\frac{n}{2}-s\right)}-\frac{\Gamma^{\prime}(s)}{\Gamma(s)}\right) d s \\
= & : I_{3_{a}}+I_{3_{b}} .
\end{aligned}
$$

For $I_{3 a}$ we repeat basically the argument of the estimate of $I_{1}$. We obtain by case differentiation of $x=\frac{1}{l_{k}\left(\mathcal{Q}^{-1}\right)}$ and $x \neq \frac{1}{l_{k}\left(\mathcal{Q}^{-1}\right)}$ the representation

$$
\begin{aligned}
I_{3_{a}}= & -i x^{\frac{n}{2}-b} \sum_{k=1}^{\infty} \frac{b_{k}\left(\mathcal{Q}^{-1}\right)}{l_{k}\left(\mathcal{Q}^{-1}\right)^{b}} \int_{-T}^{T}\left(x \cdot l_{k}\left(\mathcal{Q}^{-1}\right)\right)^{i t} d t \\
= & -i x^{\frac{n}{2}} \delta_{l_{k}\left(\mathcal{Q}^{-1}\right), \frac{1}{x}} \cdot b_{k}\left(\mathcal{Q}^{-1}\right) \cdot 2 T \\
& +O\left(x^{\frac{n}{2}-b} \sum_{k=1}^{\infty} \frac{b_{k}\left(\mathcal{Q}^{-1}\right)}{l_{k}\left(\mathcal{Q}^{-1}\right)^{b}} \cdot \min \left\{T, \frac{1}{\left|\log \left(x \cdot l_{k}\left(\mathcal{Q}^{-1}\right)\right)\right|}\right\}\right) .
\end{aligned}
$$


Again for $\frac{1}{l_{k}\left(\mathcal{Q}^{-1}\right)} \leq \frac{x}{2}$ or $\frac{1}{l_{k}\left(\mathcal{Q}^{-1}\right)} \geq 2 x$ we have $\left|\log \left(x \cdot l_{k}\left(\mathcal{Q}^{-1}\right)\right)\right| \gg 1$ and the error term on the right hand side in this case is

$$
x^{\frac{n}{2}-b} \sum_{k=1}^{\infty} \frac{b_{k}\left(\mathcal{Q}^{-1}\right)}{l_{k}\left(\mathcal{Q}^{-1}\right)^{b}} \ll x^{\frac{n}{2}-b} .
$$

Now there exists a $\beta \in \mathbb{N}$ with $\frac{1}{l_{\beta+1}\left(\mathcal{Q}^{-1}\right)}<x<\frac{1}{l_{\beta}\left(\mathcal{Q}^{-1}\right)}$. Hence, by renewed application of Lemma 6.1, the part where $\frac{x}{2}<\frac{1}{l_{k}\left(\mathcal{Q}^{-1}\right)}<x$ becomes

$$
\begin{aligned}
& \sum_{\frac{1}{2} x<\frac{1}{l_{k}\left(\mathcal{Q}^{-1}\right)}<x}\left|b_{k}\left(\mathcal{Q}^{-1}\right)\right| \cdot \min \left\{T, \frac{1}{\left|\log \left(x \cdot l_{k}\left(\mathcal{Q}^{-1}\right)\right)\right|}\right\} \\
& \ll \#\left\{k \in \mathbb{N}: 1>\frac{1}{l_{k}\left(\mathcal{Q}^{-1}\right)}>\frac{x}{2}\right\} \cdot\left(\frac{1}{l_{\beta+1}\left(\mathcal{Q}^{-1}\right)}\right)^{B^{\prime}} \cdot \min \left\{T, \frac{x}{x-\frac{1}{l_{\beta+1}\left(\mathcal{Q}^{-1}\right)}}\right\} \\
& \ll \#\left\{k \in \mathbb{N}: 1>\frac{1}{l_{k}\left(\mathcal{Q}^{-1}\right)}>\frac{x}{2}\right\} \cdot x^{-B^{\prime}} \cdot \min \left\{T, \frac{x}{\langle x\rangle}\right\}
\end{aligned}
$$

for a constant $B^{\prime}>0$. Now we can estimate $\#\left\{k \in \mathbb{N}: 1>\frac{1}{l_{k}\left(\mathcal{Q}^{-1}\right)}>\frac{x}{2}\right\}$ analogously by $\frac{2}{x} \cdot \mathrm{m}\left(\mathcal{Q}^{-1}\right)^{h_{2}} \cdot h_{2}$ with

$$
h_{2}=\frac{-\log x+\log 2}{\log \left(\frac{\mathrm{m}\left(\mathcal{Q}^{-1}\right)+1}{\mathrm{~m}\left(\mathcal{Q}^{-1}\right)}\right)} .
$$

Therefore setting

$$
D_{2}:=\left[\frac{-\log \mathrm{m}\left(\mathcal{Q}^{-1}\right)}{\log \left(\frac{\mathrm{m}\left(\mathcal{Q}^{-1}\right)+1}{\mathrm{~m}\left(\mathcal{Q}^{-1}\right)}\right)}\right]-2
$$

we obtain

$$
I_{3 a}=-i x^{\frac{n}{2}} \delta_{l_{k}\left(\mathcal{Q}^{-1}\right), \frac{1}{x}} \cdot b_{k}\left(\mathcal{Q}^{-1}\right) \cdot 2 T+O\left(x^{-B^{\prime}} \cdot x^{D_{2}} \cdot \min \left\{T, \frac{x}{\langle x\rangle}\right\}\right) .
$$

It remains to look at $I_{3 b}$ with the decomposition

$$
\begin{aligned}
I_{3 b} & =\left\{\int_{\frac{n}{2}-b-i}^{\frac{n}{2}-b+i}+\int_{\frac{n}{2}-b+i}^{\frac{n}{2}-b+i T}+\int_{\frac{n}{2}-b-i T}^{\frac{n}{2}-b-i}\right\} x^{s}\left(c_{3}-\frac{\Gamma^{\prime}\left(\frac{n}{2}-s\right)}{\Gamma\left(\frac{n}{2}-s\right)}-\frac{\Gamma^{\prime}(s)}{\Gamma(s)}\right) d s \\
& =: L_{1}+L_{2}+L_{3}
\end{aligned}
$$

Now $L_{1}$ is clearly $O\left(x^{\frac{n}{2}-b}\right) . L_{2}$ we decompose further according to 


$$
\begin{aligned}
L_{2}= & \int_{\frac{n}{2}-b+i}^{\frac{n}{2}-b+i T} x^{s}\left[c_{3}-\log s+O\left(\frac{1}{|s|}\right)-\log \left(\frac{n}{2}-s\right)+O\left(\frac{1}{\left|\frac{n}{2}-s\right|}\right)\right] d s \\
= & \int_{\frac{n}{2}-b+i}^{\frac{n}{2}-b+i T} x^{s}\left[c_{3}+O\left(\frac{1}{|s|}\right)+O\left(\frac{1}{\left|\frac{n}{2}-s\right|}\right)\right] d s \\
& -\int_{\frac{n}{2}-b+i}^{\frac{n}{2}-b+i T} x^{s}\left[\log s+\log \left(\frac{n}{2}-s\right)\right] d s \\
= & : L_{2_{a}}+L_{2_{b}} .
\end{aligned}
$$

We get

$$
\begin{aligned}
L_{2_{a}} & =\int_{\frac{n}{2}-b+i}^{\frac{n}{2}-b+i T} x^{s}\left[c_{3}+O\left(\frac{1}{|s|}\right)+O\left(\frac{1}{\left|\frac{n}{2}-s\right|}\right)\right] d s \\
& =i x^{\frac{n}{2}-b} \int_{1}^{T} x^{i t}\left[c_{3}+O\left(\frac{1}{\left|\frac{n}{2}-b+i t\right|}\right)+O\left(\frac{1}{|b-i t|}\right)\right] d t \\
& =O\left(x^{\frac{n}{2}-b} \cdot \log T\right),
\end{aligned}
$$

as well as for the remainder part

$$
\begin{aligned}
L_{2_{b}} & =-\int_{\frac{n}{2}-b+i}^{\frac{n}{2}-b+i T} x^{s}\left[\log s+\log \left(\frac{n}{2}-s\right)\right] d s \\
& =-i x^{\frac{n}{2}-b} \int_{1}^{+T} x^{i t}\left[\log \left(\frac{n}{2}-b+i t\right)+\log (b-i t)\right] d t .
\end{aligned}
$$

Applying once more partial integration we arrive at

$$
\begin{aligned}
L_{2_{b}}= & \left.\frac{x^{i t}}{i \log x}\left[\log \left(\frac{n}{2}-b+i t\right)+\log (b-i t)\right]\right|_{1} ^{T} \\
& -\int_{1}^{+T} \frac{x^{i t}}{\log x}\left(\frac{1}{\frac{n}{2}-b+i t}-\frac{1}{b-i t}\right) d t=O\left(\frac{x^{\frac{n}{2}-b}}{|\log x|} \cdot \log T\right)
\end{aligned}
$$

The same argument yields $L_{3}=O\left(\frac{x^{\frac{n}{2}-b}}{|\log x|} \cdot \log T\right)$.

Putting all pieces together and setting $\tilde{B}=\max \left\{B, B^{\prime}\right\}+\max \left\{D_{1}, D_{2}\right\}$ we obtain 


$$
\begin{aligned}
I_{1}= & 2 i \cdot \delta_{l_{k}(\mathcal{Q}), x} \cdot b_{k}(\mathcal{Q}) \cdot T+O\left(x^{\tilde{B}} \cdot \min \left(T, \frac{x}{\langle x\rangle}\right)\right), \\
I_{2}= & O\left(x^{\tilde{B}} \log T\right) \mathbf{1}_{\{x>1\}}+O\left(x^{\frac{n}{2}-b} \log T\right) \mathbf{1}_{\{0<x<1\}}, \\
I_{3}= & -i x^{\frac{n}{2}} \delta_{l_{k}\left(\mathcal{Q}^{-1}\right), \frac{1}{x}} \cdot b_{k}\left(\mathcal{Q}^{-1}\right) \cdot 2 T+O\left(x^{-\tilde{B}} \cdot \min \left(T, \frac{x}{\langle x\rangle}\right)\right) \\
& +O\left(\frac{x^{\frac{n}{2}-b}}{|\log x|} \cdot \log T\right), \\
I_{4}= & O\left(x^{\tilde{B}} \log T\right) \mathbf{1}_{\{x>1\}}+O\left(x^{\frac{n}{2}-b} \log T\right) \mathbf{1}_{\{0<x<1\}},
\end{aligned}
$$

which concludes the proof of Theorem 6.2.

\subsection{Discrepancy for zero ordinates of Epstein zeta-functions}

Based on Theorem 6.2 we are now in a position to state the main result of this chapter.

Theorem 6.3. Let $z \neq 1$ be a positive real number. Let the sequence $\gamma$ range through the set of positive imaginary parts of the nontrivial zeros of the Epstein zeta-function $\zeta(s ; \mathcal{Q})$ in ascending order and let

$$
\sum_{|\gamma|<T}\left|\beta-\frac{n}{4}\right|=O\left(\frac{T \log T}{\log \log T}\right) .
$$

Let $D_{T}$ be the discrepancy of the sequence $\left(\frac{\log z}{2 \pi} \gamma\right)_{\gamma}$. Then, as $T \rightarrow \infty$

$$
D_{T} \ll \frac{|\log z|}{\log \log \log T}
$$

Proof: According to (5.18) we start with

$$
\sum_{|\gamma|<T}\left|x^{\left(\frac{n}{4}+i \gamma\right)}-x^{(\beta+i \gamma)}\right| \leq \max \left\{x^{\beta}, x^{\frac{n}{4}}\right\}|\log x| \sum_{|\gamma|<T}\left|\beta-\frac{n}{4}\right|,
$$

hence, using the density assumption (6.3), we get

$$
\sum_{|\gamma|<T} x^{\left(\frac{n}{4}+i \gamma\right)}=\sum_{|\gamma|<T} x^{(\beta+i \gamma)}+O\left(\max \left\{x^{\beta}, x^{\frac{n}{4}}\right\}|\log x| \cdot \frac{T \log T}{\log \log T}\right) .
$$

Now inserting the asymptotic of Theorem 6.2 and dividing by $x^{\frac{n}{4}}$ we obtain 


$$
\begin{aligned}
\sum_{|\gamma|<T} x^{i \gamma}= & \frac{T}{\pi}\left(\delta_{l_{k}(\mathcal{Q}), x} \cdot b_{k}(\mathcal{Q})-x^{\frac{n}{2}} \delta_{l_{k}\left(\mathcal{Q}^{-1}\right), \frac{1}{x}} \cdot b_{k}\left(\mathcal{Q}^{-1}\right)\right) \\
& +O\left(x^{\tilde{B}} \cdot \frac{T \log T}{\log \log T}\right) \mathbf{1}_{\{x>1\}}+O\left(x^{-\tilde{B}} \cdot \frac{T \log T}{\log \log T}\right) \mathbf{1}_{\{0<x<1\}} .
\end{aligned}
$$

Here the latter two terms absorb all error terms occurring in Theorem 6.2. Now we set $x=z^{h}$ with a positive $z \neq 1$ and $h$ being a positive integer. Dividing by the number of zeros $N(T ; \mathcal{Q})$, which is $O(T \log T)$, we obtain

$$
\begin{aligned}
& \frac{1}{N(T ; \mathcal{Q})} \sum_{|\gamma|<T} e^{i h \log z \gamma} \\
& =O\left(\frac{1}{\log T} \cdot \frac{1}{\pi}\left(\delta_{l_{k}(\mathcal{Q}), z^{h}} \cdot b_{k}(\mathcal{Q})-z^{\frac{h n}{2}} \delta_{l_{k}\left(\mathcal{Q}^{-1}\right), \frac{1}{z^{h}}} \cdot b_{k}\left(\mathcal{Q}^{-1}\right)\right)\right) \\
& +O\left(z^{h \tilde{B}} \cdot \frac{1}{\log \log T}\right) \mathbf{1}_{\left\{z^{h}>1\right\}}+O\left(z^{-h \tilde{B}} \cdot \frac{1}{\log \log T}\right) \mathbf{1}_{\left\{0<z^{h}<1\right\}}
\end{aligned}
$$

We continue by looking at the terms containing $z^{h}$ and case differentiate. For $z^{h}>1$ we consider the case $z^{h}=l_{k}(\mathcal{Q})$ and get

$$
\delta_{l_{k}(\mathcal{Q}), z^{h}} \cdot b_{k}(\mathcal{Q})-z^{\frac{h n}{2}} \delta_{l_{k}\left(\mathcal{Q}^{-1}\right), \frac{1}{z^{h}}} \cdot b_{k}\left(\mathcal{Q}^{-1}\right)=b_{k}(\mathcal{Q}) \ll z^{h \tilde{B}}
$$

due to Lemma 6.1. Another application of Lemma 6.1 to the case $0<z^{h}<1$, where we consider $z^{h}=\frac{1}{l_{k}\left(\mathcal{Q}^{-1}\right)}$, yields

$$
\delta_{l_{k}(\mathcal{Q}), z^{h}} \cdot b_{k}(\mathcal{Q})-z^{\frac{h n}{2}} \delta_{l_{k}\left(\mathcal{Q}^{-1}\right), \frac{1}{z^{h}}} \cdot b_{k}\left(\mathcal{Q}^{-1}\right)=z^{\frac{h n}{2}} b_{k}\left(\mathcal{Q}^{-1}\right) \ll z^{-h \tilde{B}} .
$$

Hence, in light of $\frac{1}{\log T}<\frac{1}{\log \log T}$ for sufficiently large $T$, we arrive at the following common bound:

$$
\begin{aligned}
\frac{1}{N(T ; \mathcal{Q})} \sum_{|\gamma|<T} e^{i h \log z \gamma}= & O\left(z^{h \tilde{B}} \cdot \frac{1}{\log \log T}\right) \mathbf{1}_{\left\{z^{h}>1\right\}} \\
& +O\left(\frac{1}{z^{h \tilde{B}}} \cdot \frac{1}{\log \log T}\right) \mathbf{1}_{\left\{0<z^{h}<1\right\}}
\end{aligned}
$$

Using this and the Erdös-Turan inequality (6.1) we obtain a bound for the discrepancy $D_{T}$ : 


$$
\begin{aligned}
D_{T} & \ll \frac{1}{m} \sum_{h=1}^{m} \frac{1}{h}\left|\frac{1}{N(T ; \mathcal{Q})} \sum_{|\gamma|<T} e^{i h \log z \gamma}\right| \\
& \ll \frac{1}{m}+\frac{1}{\log \log T} \sum_{h=1}^{m} \frac{\max \left\{z^{h \tilde{B}}, z^{-h \tilde{B}}\right\}}{h} .
\end{aligned}
$$

Once more we distinguish $z^{h}>1$ and $0<z^{h}<1$. We use the general estimate

$$
\sum_{n=1}^{m} \omega^{n} \ll \frac{\omega^{m}}{m}
$$

which holds for any real $\omega>1$. Applying it to $\omega=z^{\tilde{B}}$ yields in case of $z^{h}>1$

$$
D_{T} \ll \frac{1}{m}+\frac{1}{\log \log T} \cdot \frac{z^{m \tilde{B}}}{m} .
$$

To optimize the parameter $m$ we observe that $1=\frac{z^{m \tilde{B}}}{\log \log T}$ implies $m=\frac{\log \log \log T}{\tilde{B} \log z}$. Hence we chose

$$
m=\left[\frac{\log \log \log T}{\tilde{B} \log z}\right]+1
$$

to obtain for $z>1, T \rightarrow \infty$

$$
D_{T} \ll \frac{1}{\left[\frac{\log \log \log T}{\tilde{B} \log z}\right]+1} \ll \frac{\tilde{B} \log z}{\log \log \log T} \ll \frac{\log z}{\log \log \log T} .
$$

Similarly for $0<z^{h}<1$ we have $\omega^{-\tilde{B}}>1$ and get

$$
D_{T} \ll \frac{1}{m}+\frac{1}{\log \log T} \cdot \frac{z^{-m \tilde{B}}}{m} .
$$

Now $1=\frac{1}{z^{m \tilde{B}} \log \log T}$ implies $m=-\tilde{B} \cdot \frac{\log \log \log T}{\log z}$, so baring in mind $\log z<0$ we take

$$
m=\left[-\tilde{B} \cdot \frac{\log \log \log T}{\log z}\right]+1
$$

to arrive at

$$
D_{T} \ll \frac{1}{\left[-\tilde{B} \cdot \frac{\log \log \log T}{\log z}\right]+1} \ll \frac{-\tilde{B} \log z}{\log \log \log T} \ll \frac{|\log z|}{\log \log \log T}
$$

for $0<z<1, T \rightarrow \infty$. Combining the two cases proves the discrepancy estimate of the Theorem, since we obtain for all $z \in \mathbb{R}^{+} \backslash\{1\}, T \rightarrow \infty$ 
6.3 Discrepancy for zero ordinates of Epstein zeta-functions

$$
D_{T} \ll \frac{|\log z|}{\log \log \log T}
$$

where the implied constant may depend on $\tilde{B}$. 



\section{References}

1. A. Akbary, M.R. Murty, Uniform distribution of zeros of Dirichlet series, in: Anatomy of integers, De Koninck, Jean-Marie (ed) at al., CRM workshop, Montreal, Canada, March 1317 2006, CRM Proceedings and lecture notes 46 (2008), 143-156

2. R.F. Arenstorf, L.L. Brewer, A study of the motion of zeros of the Epstein Zeta function associated to $m^{2}+y^{2} n^{2}$ as $y$ varies from 1 to $\sqrt{6}$, Computers Math. Applic. Vol. 265 (1993), 57-69

3. S. Baluyot, S.M. Gonek, Explicit formulae and discrepancy estimates for $a$-points of the Riemann zeta-function, Pacific Journal of Math. 303 (2019), 47-71

4. P.T. Bateman, E. Grosswald, On Epstein's zeta-function, Acta. Arith. 9 (1964), 365-373

5. V. Bergström, Einige Bemerkungen zur Theorie der diophantischen Approximation, Fysiogr. Sälsk. Lund. Vörh. 6 no.13 (1964), 1-19

6. E. Bombieri, D.A. Hejhal, On the distribution of zeros of linear combinations of Euler products, Duke Math. J. 80 (1995), 821-862

7. E. Bombieri, J. Müller, On the zeros of certain Epstein zeta functions, Forum Math. 20(2) (2008), 359-385

8. J.P. Buhler, R.E. Crandall, Elementary function expansions for Madelung constants, J. Phys. Series A. 20 (1987), 5497-5510

9. S. Chowla, A. Selberg, On Epstein's zeta-function I, Proc. Nat. Acad. Sci. U.S.A. 35 (1949), 371-374

10. J.H. Conway, N.J.A. Slonne, Sphere packings, lattices, and groups, Springer, 1993, 2nd ed.

11. H. Davenport, H. Heilbronn, On the zeros of certain Dirichlet series I, II, Journal London Math. Soc 11 (1936), 181-185, 307-312

12. H. Davenport, Multiplicative Number Theory, Graduate Texts in Mathematics, 3rd ed., Springer, New York, 2000

13. M. Deuring, Zetafunktionen quadratischer Formen, Journal Reine Ang. Math. 172 (1935), 226-252

14. S. Egami, K. Matsumoto, Convolutions of the von Mangoldt function and related Dirichlet series, in Number Theory World Sci. Publ. 2007, 1-23

15. E. Elizalde, A. Romeo, Regularization of general multidimensional Epstein zeta-functions, Rev. Math. Phys., 1 (1989), 113-128 
16. E. Elizalde, Ten Physical Applications of Spectral Zeta Functions, 2nd ed., Lecture Notes in Physics 855, Springer, Heidelberg, 2012

17. P.D.T.A. Elliott, The Riemann zeta function and coin tossing, Journal Reine Ang. Math. 254 (1972), 100-109

18. P. Epstein, Zur Theorie allgemeiner Zetafunctionen, Math. Annal. 56 (1903), 615-644

19. P. ERdös, P. TurÁn On a problem in the theory of uniform distribution I, II, Indag. Math 10 (1948), 370-378, 406-413

20. O.M. Fomenko, Order of the Epstein zeta-function in the critical strip, J. Math. Sci. 110(6) (2002), 3150-3163

21. A. FujII, On the zeros of the Epstein zeta-functions, J. Math. Kyoto Univ. 36(4) (1996), 697-770

22. A. FuJII, Uniform distribution of the zeros of the Riemann zeta-function and the mean value theorems of Dirichlet L-functions. II, in Analytic number theory; Tokyo 1988 Lecture Notes in Math. 1434 Springer 1990, 103-125

23. A. Fujil, An additive theory of the zeros of the Riemann zeta-function, Proc. Japan Acad. 66 (1990), 105-108

24. A. FujiI, On a theorem of Landau, Proc. Japan Acad. 65 (1989), 51-54

25. A. Fu JiI, On a theorem of Landau, II Proc. Japan Acad. 66 (1990), 291-296

26. A. FuJil, Uniform distrbution of the zeros of the Riemann zeta-function and the mean value theorem of Dirichlet L-functions, in: Analytic number Theory, Lecture Notes in Mathematics, vol. 1434, Springer, New York (1990), 103-125. , Proc. Japan Acad. 65 (1989), 51-54

27. A. Fujil, Some problems on Diophantine approximation in the theory of the Riemann zeta function III, Comment Math. Univ. St. Pauli 42 (1993), no. $2,161-187$

28. S.M. Gonek, A formula of Landau and mean values of $\zeta(s)$, in Topics in Analytic Number Theory ed by S.W. Graham and J.D. VaAler Univ. Texas Press 1985, 92-97

29. S.M. Gonek, An explicit formula of Landau and its applications to the theory of zeta-functions, Contemp. Math. 143 (1993), 395-413

30. S.M. Gonek, Y. Lee, Zero-density estimates for Epstein zeta-functions, Quart. J. Math. 68 (2017), 301-344

31. R. Garunkštis, J. Steuding, R. Šimėnas, The a-points of the Selberg zeta-function are uniformly distributed modulo one, Illinois Journal of Math., Vol. 158 (2014), 207-218

32. F. Halter-Koch, Quadratic Irrationals, CRC Press 2013

33. G.H. Hardy, M. Riesz, The general theory of Dirichlet's series, Cambridge University Press, Cambridge, 1915

34. E. Hecke, Über Modulfunktionen und die Dirichletschen Reihen mit Eulerscher Produktentwicklung. I, II, Math. Ann. 114 (1937), 1-28,316-351

35. D.A. Hejhal, Zeros of Epstein zeta functions and supercomputers, in: Proc. Int. Congr. Math., Berkeley/Calif. 1986, Vol. 2, (1987), 1362-1384

36. E. Hlawka, Über die Gleichverteilung gewisser Folgen, welche mit den Nullstellen der Zetafunktion zusammenhängen, Österr. Akad. Wiss., Math.-Naturw. Kl. Abt. II, (1975), 459-471; in: Selecta (Springer Collected Works in Mathematics), P. Gruber et al. (Eds.), Springer, Berlin, (1990), 385-397.

37. H. Iwaniec, Topics in classical automorphic forms, Amer. Math. Soc., Providence, 171997 
38. J. Kaczorowski, A. Languasco, A. Perelli, A note on Landau's formula, Funct. Approx. Comment. 28 (2000), 173-186

39. A.A. Karatsuba, S.M. Voronin, The Riemann zeta-function, de Gruyter 1992

40. M. Koecher, A. Krieg, Elliptische Funktionen und Modulformen, Springer 1998

41. A.N. Kolmogorov, S.V. Fomin, Elements of the Theory of Functions and Functional Analysis, (Russian), 3th ed., Izd. Nauka, Moscow, 1972 (Zbl 0235.46001; 4th ed. MR0435771 (55 8728))

42. L. Kuipers, H. Niederreiter, Uniform distribution of sequences, John Wiley \& Sons, New York 1974

43. E. Landau, Über die Nullstellen der Zetafunktion, Math. Ann. 71 (1912), 548-564

44. E. Landau, Über den Wertevorrat von $\zeta(s)$ in der Halbebene $\varrho>$ 1, Nachrichten von der Gesellschaft der Wissenschaften zu Göttingen, Mathematisch-Physikalische Klasse Volume (1933), 81-91

45. Y. LeE, On the zeros of Epstein zeta-functions, Forum Math. 26 (2014), $1807-1836$

46. Y. LEE, On the zeros of Epstein zeta-functions near the critical line, Jour. Math. Analysis Appl. 487(1) (2020)

47. N. Levinson, Almost all roots of $\zeta(s)=a$ are arbitrarily close to $\sigma=1 / 2$, Proc. Nat. Acad. Sci. U.S.A. 72 (1975), 1322-1324

48. J. E. Littlewood, On the zeros of the Riemann zeta-function, Proc. Cambridge Phil. Soc. 22 (1924), 295-318

49. W. MÜLLER, The mean square of Dirchlet series associated with automorphic forms, Monatshefte Mathematik 113 (1992), 121-159

50. M.R. Murty, V.K. Murty, Strong multiplicity one for Selberg's class, C. R. Acad. Sci. Paris Ser. I Math., 319 (1994), 315-320

51. Y. Мотонаsнi, A note on the mean value of the zeta and $L$-functions. II, Proc. Japan Acad. Ser. A Math., 61 (1985), 313-316

52. T. NAkAmura, L. PańkOwski, On zeros and $c$-values of Epstein zetafunctions, Siauliai. Math. Semin. 8 (16) (2013), 181-195

53. W. NARKIEWICZ, Elementary and analytic theory of algebraic numbers, Springer 2004

54. H.S.A. Potter, E.C. Titchmarsh, The zeros of Epstein's zeta-functions, Proc. London Math. Soc. 39 (1935), 372-384

55. H.A. Rademacher, Fourier Analysis in Number Theory, Symposium on Harmonic Analysis and Related Integral Transforms (Cornwell Univ.,Ithaca,N.Y.,1956) in: Collected Papers of Hans Rademacher, Vol.II, pp. 434-458, Massachusetts Inst. Tech., Cambridge, Mass., 1974

56. M. Rehberg A discrepancy estimate for the a-points of the Riemann zeta function, in: Anal. Probab. Methods Number Theory, Proceedings of the Sixth International Conference in Honour of J. Kubilius, Palanga 2016, A. Dubickas et al. (Eds.), TEV, Vilnius 2017, 165-178

57. I.S. Rezvyakova, On the zeros of the Epstein zeta-function on the critical line, Russ. Math. Surv. 70 (2015), 785-787, transl. from Usp. Mat. Nauk 70 (2015), 213-214

58. K.F. Rотн On irregularities of distribution, Mathematika 1 (1954), 73-79.

59. C. SCHMEller On the uniform distribution of zero ordinates of Epstein zeta-functions, Lithuanian Math. Journal 58 (2018), 198-211. 
60. W.M. Schmidt Irregularities of distribution VII, Act. Arith. 21 (1972), $45-50$.

61. A. Selberg, On the zeros of the Riemann zeta-function, Skr. Norske Vid. Akad. Oslo 10 (1942), 1-59

62. C.L. Siegel, Contributions to the theory of the Dirichlet L-series and the Epstein zeta-functions, Ann. of Math., 44(2) (1943), 143-172

63. C.L. Siegel, Advanced Analytic Number Theory 2nd ed., Tata Institute of Fundamental Research, Bombay 1980

64. H.M. Stark, On the zeros of Epstein zeta-functions, Mathematika 14 (1967), 47-55

65. J. Steuding, On the zero-distribution of Epstein zeta-functions, Math. Annalen 333 (2005), 689-697

66. J. Steuding, On the value-distribution of Epstein zeta-functions, $P u b$ licacions Mat., Proceedings 'Primeras Jornadas de Teoria de Números', Vilanova i la Geltrú (Barcelona) 2005, special volume, (2007), 221-242

67. J. Steuding, The roots of the equation $\zeta(s)=a$ are uniformly distributed modulo one, Analytic and probabilistic methods in number theory, (2011), 243-249

68. J. Steuding, One hundred years uniform distribution modulo one and recent applications to Riemann's zeta function, Topics in mathematical Analysis and Applications, Springer Volume 94 (2014), 659-698

69. J. Steuding, Value distribution of L-functions, Lecture Notes in Mathematics 1877, Springer, 2007

70. O. Strauch, Š.PorubSkÝ, Distribution of Sequences, A Sampler, Peter Lang, Europäischer Verlag der Wissenschaften, electronic revised version 2016, 2005

71. A. Terras, The minima of quadratic forms and the behaviour of Epstein and Dedekind zeta-functions, J. Number Theory 12(1980), 258-272

72. E.C. Titchmarsh, The theory of the Riemann zeta-function, Oxford University Press 1986, 2nd ed., revised by D.R. Heath-Brown

73. I. TravĔnec, L. S̆AmaJ, Generation of off-critical zeros for hypercubic Epstein zeta-functions, preprint, Slovak. Academy of Sciences (2019)

74. T. Van Aardenne-Ehrenfest: Proof of the impossibility of a just distribution of an infinite sequence of points over an interval, Indag. Math. 7 (1945), 71-76.

75. J.G. Van der Corput, C. Pisot, Sur la discré pance modulo un, Indag. Math. 1 (1939), 260-269

76. I.M. VinOGRADOv, Representation of an odd number as the sum of three primes, Doklady Akad. Nauk SSSR 15(1937), 291-294 (Russian)

77. S.M. Voronin, The zeros of zeta-functions of quadratic forms (in Russian), Tr. Math. Inst. Steklova 142(1976), 135-147

78. H. WEYL, Sur une application de la théorie des nombres à la mécanique statistique et la théorie des pertubations, L'Enseign. math 16 (1914), 455467

79. H. Weyl, Über die Gleichverteilung von Zahlen mod. Eins, Math. Ann., 77 (1916), 313-352

80. H. Weyl, Über ein Problem aus dem Gebiet der diophantischen Approximationen , Gött. Nachr. (1914), 234-244

81. Q. Zhang, Integral mean values of modular $L$-functions, J. Number Theory 115 (2005), 100-122 
References

82. Q. ZhANG, Integral mean values of Maass L-functions, Internat. Math. Res. Notices (2006), Article ID 41417, 1-19 